\title{
Der Status quo des öffentlichen Einkaufs
}

Folgendes Kapitel präsentiert zunächst Zahlen und Fakten zum öffentlichen Einkauf, gefolgt von den rechtlichen Rahmenbedingungen der Vergabe. Die organisatorische Umsetzung zeigt Organisationsformen im öffentlichen Einkauf sowie die Bedeutung des Einkaufsprozesses in einer gesamthaften Betrachtung auf. Die technische Durchdringung fokussiert auf den Stand des elektronischen Einkaufs als Voraussetzung für eine mögliche Öffnung. Anschließend wird die Bedeutung von Transparenz, Korruption, Partizipation und Kollaboration dargelegt.

Anliegen ist, ein möglichst konkretes Bild vom gegenwärtigen Zustand des öffentlichen Einkaufs zu zeichnen, um eine Basis für die nachfolgenden empirischen und analytischen Abschnitte der Studie zu schaffen.

\subsection{Statistische Erhebungen zum öffentlichen Beschaffungsvolumen}

\subsubsection{Das öffentliche Beschaffungsvolumen Deutschlands}

Im folgenden Abschnitt werden die vorliegenden Erhebungen in chronologischer Reihenfolge ihres Erscheinens erläutert.

Das BMBF beauftragte im Jahr 2008 die Wegweiser GmbH, die TU Berlin und Orrick Hölters \& Elsing ${ }^{1}$ mit einer Untersuchung zur ,innovativere[n] Beschaffung“. Die Ergebnisse sind nachzulesen in der Publikation „Einkäufer

\footnotetext{
${ }^{1}$ Im Jahr 2013 benannten sich die deutschen Standorte der Kanzlei Örrick Hölters \& Elsing in Orrick, Herrington \& Sutcliffe um (Wnuck, 2008). 
Staat als Innovationstreiber - Entwicklungspotenziale und Handlungsnotwendigkeiten für eine innovativere Beschaffung im öffentlichen Auftragswesen Deutschlands". Die Autoren versuchen das öffentliche Beschaffungsvolumen für die Jahre 2001 bis 2006 sowie den innovationsrelevanten Anteil am Beschaffungsvolumen $\mathrm{zu}$ bestimmen. Insgesamt ermitteln sie Beschaffungsvolumina zwischen etwa 210 Milliarden Euro (2001) und etwa 247 Milliarden Euro (2006). Die Erhebung greift auf Daten des statistischen Bundesamtes und Haushaltspläne aus dem Jahr 2008 zurück; Sachaufwände und Sachinvestitionen gehen in die Berechnung ein. Für Fonds, Einrichtungen und Unternehmen (FEU) wird auf Basis der Angaben zum Materialaufwand innerhalb der Jahres- und Geschäftsberichte auf ein Beschaffungsvolumen hochgerechnet. Eine weitere Detaillierung des Vorgehens wird im Rahmen des Wegweiser-Berichts allerdings nicht vorgenommen (Lorenz et al., 2009).

Die OECD gibt etwa alle zwei Jahre die Publikation „Government at a Glance“ heraus, in Abbildung 6.1 und Tabelle 6.1 sind die dort aufgeführten Zahlen zum Beschaffungsvolumen von 2007 bis 2015 zusammengefasst. Als einer von zwölf Bereichen wird die öffentliche Beschaffung über die Mitgliedsstaaten hinweg verglichen. Das Gesamtbeschaffungsvolumen, die strategische Beschaffung, die elektronische Beschaffung und die zentralisierte Beschaffung sind jeweils mit Indikatoren hinterlegt. Die OECD bedient sich einer annähernd gleichen Methodik wie folgend die EU, somit sind Abweichungen zwischen beiden Erhebungen nur marginal (OECD, 2009a; 2011; 2013; 2015; 2017).

\section{Geschätztes öffentliches Beschaffungsvolumen in Deutschland gemäß OECD (in Milliarden €)}

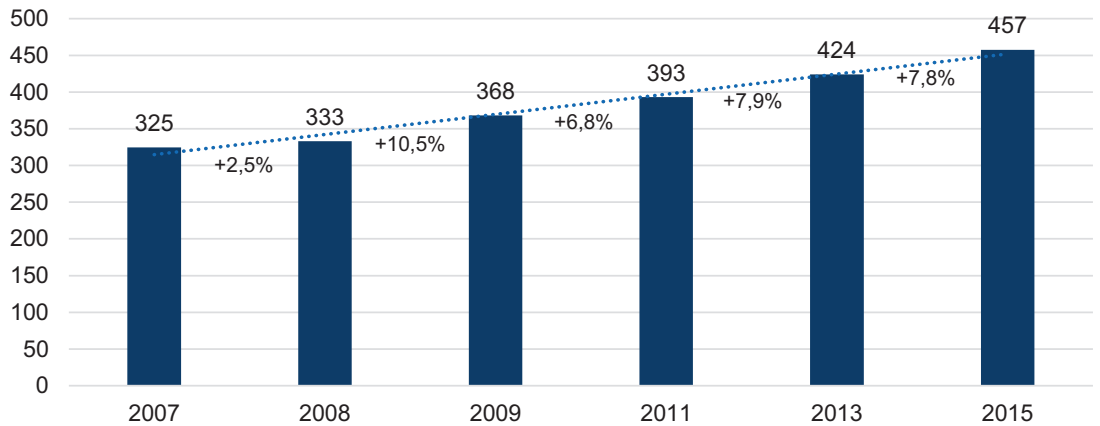

Abbildung 6.1 Geschätztes öffentliches Beschaffungsvolumen in Deutschland laut OECD (in Milliarden Euro) (in Anlehnung an OECD, 2009a; 2011; 2013; 2015; 2017) 
Tabelle 6.1 Geschätztes öffentliches Beschaffungsvolumen in Deutschland gemäß OECD (in Milliarden Euro und als \% vom BIP) (in Anlehnung an OECD, 2009a; 2011; 2013; 2015; 2017; Statistisches Bundesamt (Destatis), 2019)

\begin{tabular}{l|c|c|c|c|c|c}
\hline Kategorie & $\mathbf{2 0 0 7}$ & $\mathbf{2 0 0 8}$ & $\mathbf{2 0 0 9}$ & $\mathbf{2 0 1 1}$ & $\mathbf{2 0 1 3}$ & $\mathbf{2 0 1 5}$ \\
\hline $\begin{array}{l}\text { Öffentliche } \\
\text { Beschaffung in } \\
\begin{array}{l}\text { Milliarden Euro } \\
\text { (geschätzt) }\end{array}\end{array}$ & 325 & 333 & 368 & 393 & 424 & 457 \\
\hline $\begin{array}{l}\% \text { am BIP in } \\
\text { Milliarden Euro }\end{array}$ & $12,9 \%$ & $13,0 \%$ & $15,0 \%$ & $14,5 \%$ & $15,0 \%$ & $15,0 \%$ \\
\hline $\begin{array}{l}\text { BIP in Milliarden } \\
\text { Euro }\end{array}$ & $2.513,23$ & $2.561,74$ & $2.460,28$ & $2.703,12$ & $2.826,24$ & $3.048,86$ \\
\hline
\end{tabular}

Für die Entwicklung des Beschaffungsmarktes kann zudem die jährliche Erhebung der EU „Public Procurement Indicators“ herangezogen werden. Sie zieht unter anderem das Gesamtbeschaffungsvolumen, das Gesamtbeschaffungsvolumen als Prozentsatz des BIP, das Volumen und die Anzahl der in der Datenbank Tenders Electronic Daily (TED) veröffentlichten Beschaffungen heran. Laut den EU Public Procurement Indicators entwickelte sich das öffentliche Beschaffungsvolumen für Deutschland von 2011 bis 2015 wie in Abbildung 6.2 und Tabelle 6.2 illustriert:

Geschätztes öffentliches Beschaffungsvolumen in Deutschland gemäß EU (in Milliarden Euro)



Abbildung 6.2 Geschätztes öffentliches Beschaffungsvolumen in Deutschland gemäß EU (in Milliarden Euro) (in Anlehnung an European Commission, 2012a; 2016e) 
Tabelle 6.2 Geschätztes öffentliches Beschaffungsvolumen in Deutschland gemäß EU (in Milliarden Euro und als \% vom BIP) (in Anlehnung an European Commission, 2012a; 2016e; Statistisches Bundesamt (Destatis), 2019)

\begin{tabular}{l|c|c|c|c|c}
\hline Kategorie & $\mathbf{2 0 1 1}$ & $\mathbf{2 0 1 2}$ & $\mathbf{2 0 1 3}$ & $\mathbf{2 0 1 4}$ & $\mathbf{2 0 1 5}$ \\
\hline $\begin{array}{l}\text { Öffentliche Beschaffung in } \\
\text { Milliarden Euro (geschätzt) }\end{array}$ & 395 & 409 & 426 & 442 & 462 \\
\hline$\%$ am BIP in Milliarden Euro & $14,7 \%$ & $14,9 \%$ & $15,2 \%$ & $15,1 \%$ & $15,2 \%$ \\
\hline BIP in Milliarden Euro & $2.693,56$ & $2.745,31$ & $2.811,35$ & $2.927,43$ & $3.030,07$ \\
\hline
\end{tabular}

Die Erhebung greift auf die in der Eurostat hinterlegten Statistiken zurück, das heißt auf Haushaltspläne und innerhalb dieser auf die beschaffungsnahen Ausgabeblöcke, zum Beispiel ,intermediate consumption“ (mittlere Verbrauchs-/ Konsumausgaben), „gross fixed capital formation“ (Bruttoanlageinvestitionen) und „social transfers in kind - purchased market production“ (Sozialleistungen, welche in Form von materiellen Gütern ausgegeben werden). Nicht enthalten in den EU Public Procurement Indicators sind Beschaffungsvolumina für Verteidigung und Sicherheit sowie FEU. In 2011 waren Leistungen staatlicher Versorgungsbetriebe noch berücksichtigt (European Commission, 2012a, S. $1 \mathrm{ff}$.), die zum Zwecke der Vergleichbarkeit mit den Folgejahren in Abbildung 6.2 jedoch herausgerechnet wurden (European Commission, 2016e).

Eßig und Schaupp führten im Jahr 2016 im Namen des Kompetenzzentrums für innovative Beschaffung (KOINNO) und des Forschungszentrums für Recht und Management öffentlicher Beschaffung (FoRMÖB) eine „Ermittlung des innovationsrelevanten Beschaffungsvolumens des öffentlichen Sektors als Grundlage für eine innovative öffentliche Beschaffung" durch. Sie nahmen eine Abschätzung des gesamten öffentlichen Beschaffungsvolumens vor, um hieraus das innovationsrelevante Beschaffungsvolumen abzuleiten. In dieser Studie werden sowohl Sozialaufwendungen als auch FEU berücksichtigt, die Daten stammen aus Haushaltsplänen sowie Geschäftsberichten. Insgesamt beläuft sich demnach für das Jahr 2016 das Beschaffungsvolumen auf 348,5 Milliarden Euro. Davon entfallen etwa 32 Milliarden Euro auf den Bund, 26,5 Milliarden Euro auf die Länder, 65 Milliarden Euro auf die Kommunen, 9,5 Milliarden Euro auf Sozialversicherungen und 215,5 Milliarden Euro auf FEU (Schaupp \& Eßig, 2016, S. 51).

\subsubsection{Verteilung der öffentlichen Vergabe auf Oberschwellen- und Unterschwellenbereich}

Nach der Erhebung im Rahmen des Projektes zur Vergabestatistik im Jahr 2014 (Baumann et al., 2014, S. 49 ff.), an welcher 194 Vergabestellen mit einem Gesamt- 
beschaffungsvolumen von 5,58 Milliarden Euro teilnahmen ${ }^{2}$, ergibt sich in der Betrachtung der Anzahlen gemäß Tabelle 6.3, dass circa $87 \%$ der Vergaben auf den Unterschwellenbereich (USB) und etwa $13 \%$ auf den Oberschwellenbereich (OSB) entfallen (die Zahlenbasis stammt aus 2013). Dies bestätigend, kommen auch die Autoren des Gutachtens zur öffentlichen Beschaffung aus 2007 zu dem Schluss, „dass Vergaben unterhalb der EU-Schwellenwerte etwa 80-90\% aller Vergaben ausmachen“ (Bundesministerium für Wirtschaft und Technologie, 2007, S. 7).

In der Aufteilung auf unterschiedliche Ebenen entfällt gemäß Tabelle 6.3 die Mehrzahl aller Aufträge auf den Kommunalbereich mit $41 \%$, gefolgt von der Landes- und Bundesebene (32\% versus $19 \%$ ).

Tabelle 6.3 Aufteilung der Vergabeverfahren insgesamt (Anzahl) (in Anlehnung an Baumann et al., 2014, S. 50)

\begin{tabular}{|c|c|c|c|c|}
\hline $\begin{array}{l}\text { Kategorie der } \\
\text { Vergabestelle }\end{array}$ & $\begin{array}{c}\text { n }(136 \\
\text { aus 194) }\end{array}$ & $\begin{array}{c}\text { Anteil an allen } \\
\text { gemeldeten } \\
\text { Vergabeverfahren } \\
\text { (nach Anzahl) }\end{array}$ & $\begin{array}{c}\text { Anteil der } \\
\text { Verfahren im } \\
\text { OSB } \\
\text { (Mittelwert; } \\
\text { nach Anzahl) }\end{array}$ & $\begin{array}{c}\text { Anteil der } \\
\text { Verfahren im } \\
\text { USB } \\
\text { (Mittelwert; } \\
\text { nach Anzahl) }\end{array}$ \\
\hline $\begin{array}{l}\text { Bund beziehungsweise } \\
\text { nachgeordnete } \\
\text { Bundesbehörde }\end{array}$ & 17 & $19 \%$ & $12 \%$ & $88 \%$ \\
\hline $\begin{array}{l}\text { Landesministerium } \\
\text { beziehungsweise } \\
\text { nachgeordnete } \\
\text { Landesbehörde }\end{array}$ & 24 & $32 \%$ & $18 \%$ & $82 \%$ \\
\hline $\begin{array}{l}\text { Gemeinde, } \\
\text { Gemeindeverband, } \\
\text { Kommunalverband, Kreis }\end{array}$ & 83 & $41 \%$ & $11 \%$ & $89 \%$ \\
\hline $\begin{array}{l}\text { Sektorenauftraggeber } \\
\text { (gem. § } 98 \text { Nr. } 4 \text { GWB) }\end{array}$ & 2 & $0 \%$ & $5 \%$ & $95 \%$ \\
\hline Andere & 10 & $0 \%$ & $21 \%$ & $79 \%$ \\
\hline Mittelwert &. &. & $13 \%$ & $87 \%$ \\
\hline
\end{tabular}

${ }^{2}$ Es ist zu beachten, dass die Repräsentativität der Erhebung gegebenenfalls nicht ausreichend ist (die Beteiligungsquote beträgt $0,6 \%$ bei 194 teilnehmenden Vergabestellen von angenommenen 30000 Vergabestellen (siehe Abschnitt 6.1) (Kröber et al., 2008, S. 34)); das Vergabevolumen mit 5,58 Milliarden Euro hat einen Anteil von etwa 1,3\% gemessen am Gesamtvolumen von etwa 424 Milliarden Euro für 2013 (OECD, 2015, S. 137). Dennoch ist anzunehmen, dass Tendenzen aufgezeigt werden können. 
Neben dem Blick auf die Anzahl ist in Ergänzung auch die Betrachtung des Volumens wichtig. Das Volumen der Aufträge verteilt sich gemäß Tabelle 6.4 zu etwa $64 \%$ auf den Unterschwellenbereich und zu etwa $36 \%$ auf den Oberschwellenbereich. Die Studie der Europäischen Kommission „Study on administrative capacity in the EU“ kommt für Deutschland sogar zu dem Schluss, dass (basierend auf Zahlen der TED aus 2013) nur $6 \%$ aller Vergaben auf den Oberschwellenbereich entfallen und Deutschland das EU-Land mit der diesbezüglich geringsten Quote sei (European Commission, 2016c, S. 89).

In der Aufteilung auf unterschiedliche Ebenen sind gemäß Tabelle 6.4 die Bundesstellen mit $37 \%$ führend, gefolgt von der Landesebene mit $34 \%$ und letztlich der Kommunalebene mit $22 \%$. Indes nennt die Studie „Kommunale Beschaffung im Umbruch - Große deutsche Kommunen auf dem Weg zu einem nachhaltigen Einkauf?" für den Kommunalbereich einen Anteil von 40-60 \% am gesamten Beschaffungsvolumen (Beck \& Schuster, 2013, S. 8). Hier gibt es also ebenfalls statistischen Klärungsbedarf.

Trotz - oder gerade aufgrund - der Unklarheiten in den Zahlen, wird die Bedeutung der Transparenz des Unterschwellenbereichs deutlich: Es handelt sich um einen Bereich, der keinesfalls zu vernachlässigen, sondern vielmehr klar zu beleuchten ist.

Tabelle 6.4 Aufteilung des Vergabevolumens insgesamt (Volumina) (in Anlehnung an Baumann et al., 2014, S. 51)

\begin{tabular}{l|c|c|c|c}
\hline $\begin{array}{l}\text { Kategorie der } \\
\text { Vergabestelle }\end{array}$ & $\begin{array}{c}\text { n (76 } \\
\text { aus } \\
\mathbf{1 9 4})\end{array}$ & $\begin{array}{c}\text { Anteil an allen } \\
\text { gemeldeten } \\
\text { Vergabeverfahren } \\
\text { (nach Volumen) }\end{array}$ & $\begin{array}{c}\text { Anteil der } \\
\text { Verfahren im } \\
\text { OSB } \\
\text { (Mittelwert; } \\
\text { nach Volumen) }\end{array}$ & $\begin{array}{c}\text { Anteil der } \\
\text { Verfahren im } \\
\text { USB } \\
\text { (Mittelwert; } \\
\text { nach Volumen) }\end{array}$ \\
\hline $\begin{array}{l}\text { Bund beziehungsweise } \\
\text { nachgeordnete } \\
\text { Bundesbehörde }\end{array}$ & 14 & $37 \%$ & $54 \%$ & $46 \%$ \\
\hline $\begin{array}{l}\text { Landesministerium } \\
\text { beziehungsweise } \\
\text { nachgeordnete }\end{array}$ & 17 & $34 \%$ & $46 \%$ & $54 \%$ \\
$\begin{array}{l}\text { Landesbehörde } \\
\text { Gemeinde, } \\
\text { Gemeindeverband, } \\
\text { Kommunalverband, Kreis }\end{array}$ & 40 & $22 \%$ & $24 \%$ & $76 \%$ \\
\hline $\begin{array}{l}\text { Sektorenauftraggeber } \\
\text { (gem. § 98 Nr. 4 GWB) }\end{array}$ & 1 & $32 \%$ & $50 \%$ & $50 \%$ \\
\hline $\begin{array}{l}\text { Andere } \\
\text { Mittelwert }\end{array}$ & 4 & $4 \%$ & $39 \%$ & $61 \%$ \\
\hline
\end{tabular}


Hierbei zu beachten ist, dass sich der kommunale Bereich über eine weit höhere Zahl von Vergabestellen erstreckt als die Landes- oder Bundesebene. Zur Struktur der Vergabestellen nehmen Rambøll und das Institut für den Mittelstand (IfM) an, dass sich die, ,angenommenen 30000 Vergabestellen zu $12 \%$ auf Bundesebene, zu $30 \%$ auf Landesebene und zu $58 \%$ auf kommunale Ebene verteilen“ (Kröber et al., 2008, S. 34). Für Vorgehensmodelle und die Definition passender Maßnahmen ist es daher wichtig, die stark fragmentierte kommunale Ebene in geeigneter Weise zu berücksichtigen.

\subsubsection{Verteilung der öffentlichen nationalen Vergaben auf Verfahrensarten}

Eine weitere Frage betrifft jene nach der Verteilung der öffentlichen nationalen Vergaben auf die unterschiedlichen Verfahrensarten. Die Verfahrensarten sind die öffentliche Ausschreibung, die freihändige Vergabe mit Teilnahmewettbewerb, die beschränkte Ausschreibung mit Teilnahmewettbewerb, die freihändige Vergabe ohne Teilnahmewettbewerb und die beschränkte Ausschreibung ohne Teilnahmewettbewerb.

$\mathrm{Zu}$ einer Antwort trägt die Studie „Kostenmessung der Prozesse öffentlicher Liefer-, Dienstleistungs- und Bauaufträge aus Sicht der Wirtschaft und der öffentlichen Auftraggeber" aus dem Jahr 2008 bei (Kröber et al., 2008). Die Autoren berechnen die Fallzahlen für nationale Vergaben anhand der Gesamtsumme aller öffentlichen Aufträge von geschätzten 262 Milliarden Euro. Von diesem Betrag werden 5,2 Milliarden Euro in Abzug gebracht für EU-Vergaben, die statistisch erhoben werden müssen. Der Anteil, welcher gemäß Bundesbauverband auf die Vergabeordnung für Bauleistungen Abschnitt A (VOB/A) entfällt, liegt bei etwa 30 Milliarden Euro, sodass für die Vergabeordnung für Dienstleistungen Abschnitt A (VOL/A) noch 226,8 Milliarden Euro verbleiben. Laut Vergabeordnung für freiberufliche Leistungen (VOF) darf unterschwellig nicht vergeben werden. Um hieraus Verfahrenszahlen zu ermitteln, mussten Kröber et al. durchschnittliche Auftragswerte schätzen und durch Expertengespräche validieren. Die Auftragswerte sind in der Studie mit 200.000 Euro bei VOB/A-Verfahren und 100.000 Euro bei VOL/A-Verfahren eingeordnet. Es ergeben sich damit pro Jahr etwa 150000 Vergaben nach VOB/A und etwa 2,27 Millionen Vergaben nach VOL/A (Kröber et al., 2008, S. 142). 
Damit lässt sich folgendes Bild für die VOB/A zeichnen:

Anzahl der Vergabeverfahren nach VOB/A in \% (2008)

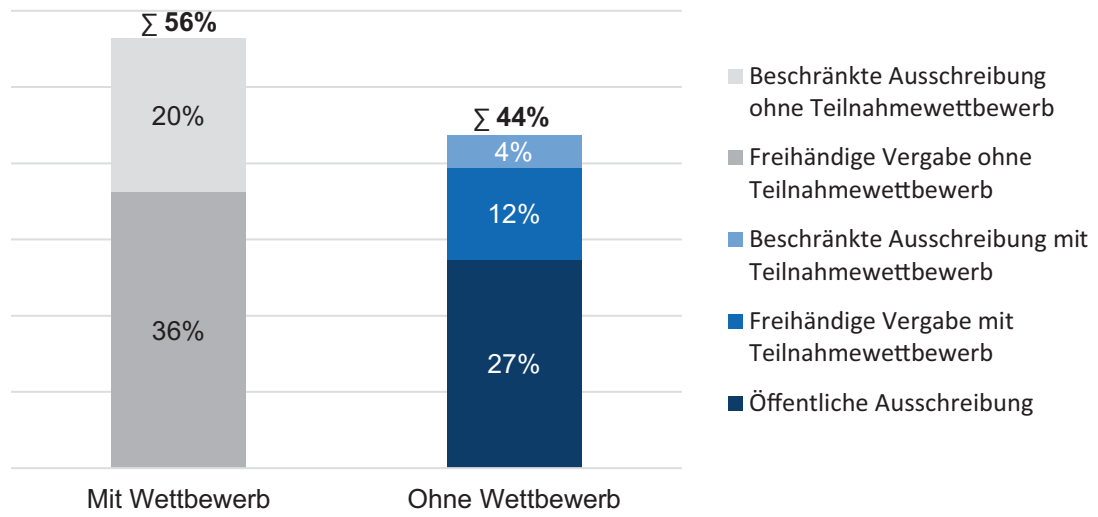

Abbildung 6.3 Anzahl Vergabeverfahren national nach VOB/A nach Verfahrensarten (2008) (in Anlehnung an Kröber et al., 2008, S. 143)

Und für die VOL/A folgt:

Anzahl der Vergabeverfahren nach VOL/A in \% (2008)

$\Sigma 77 \%$

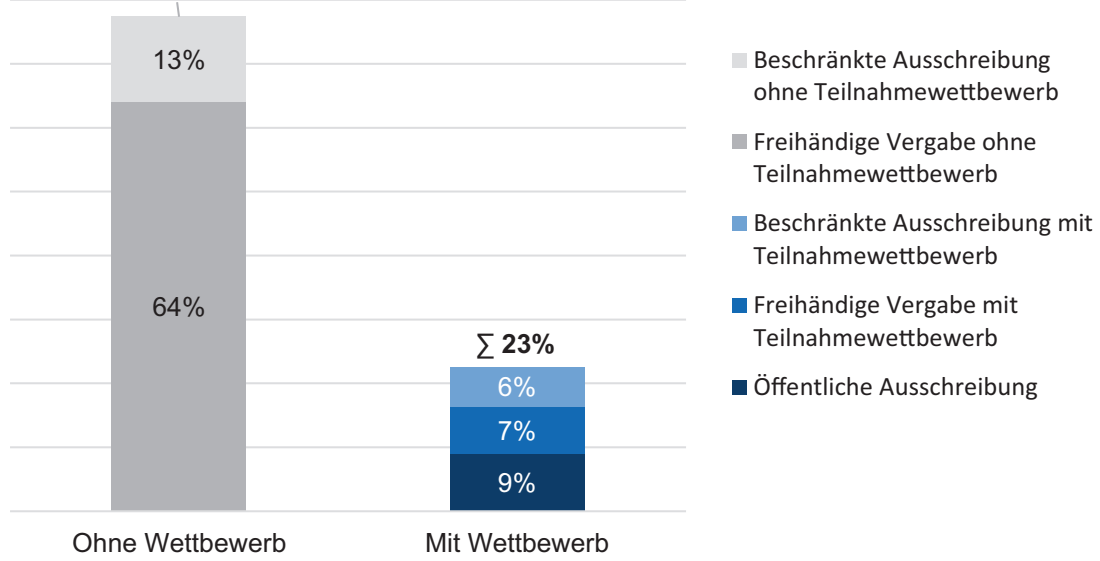

Abbildung 6.4 Anzahl Vergabeverfahren national nach VOL/A nach Verfahrensarten (2008) (in Anlehnung an Kröber et al., 2008, S. 143) 
Daraus resultiert, dass der Anteil der öffentlichen Ausschreibung gemäß Abbildung 6.3 bei immerhin $27 \%$ für die VOB/A liegt (zusammen mit der beschränkten Ausschreibung mit Teilnahmewettbewerb sowie der freihändigen Ausschreibung mit Teilnahmewettbewerb bei knapp $44 \%$ ), der Anteil für die VOL/A gemäß Abbildung 6.4 jedoch nur bei knapp $9 \%$ (zusammen mit der beschränkten Ausschreibung mit Teilnahmewettbewerb sowie der freihändigen Ausschreibung mit Teilnahmewettbewerb bei $23 \%$ ). Umgekehrt lag der Anteil der nicht-öffentlichen Verfahren für die VOB/A (beschränkte Ausschreibung ohne Teilnahmewettbewerb und freihändige Vergabe ohne Teilnahmewettbewerb) gemäß Abbildung 6.3 bei $56 \%$ und für die VOL/A gemäß Abbildung 6.4 bei $77 \%$ (Kröber et al., 2008, S. 142 ff.). Zu berücksichtigen ist, dass diese Zahlen mehr als zehn Jahre alt sind. Inwiefern sie eine Näherung an die Realität darstellen, lässt sich ohne Weiteres nicht überprüfen.

In der Studie „Einkäufer Staat als Innovationstreiber“, die sich ebenfalls auf Zahlen aus dem Jahr 2008 bezieht, wurden ähnliche Ergebnisse konstatiert, wobei dort nach föderalen Ebenen, aber nicht nach Vergabeordnungen unterschieden wurde: Das am wenigsten formalisierte und den Wettbewerb am stärksten beschränkende Vergabeverfahren der freihändigen Vergabe wird nach Abbildung $6.5 \mathrm{im}$ nationalen unterschwelligen Bereich mit durchschnittlich knapp $41 \%$ am häufigsten genutzt. Insbesondere auf der Ebene des Bundes wurde über die Hälfte der 2008 vergebenen Aufträge hierüber abgewickelt. Ein ergänzender Teilnahmewettbewerb im Vorfeld der freihändigen Vergabe erfolgte bei weiteren $7 \%$ der Studienteilnehmer. Die öffentliche Ausschreibung kommt demgegenüber lediglich auf einen Anteil von etwa $23 \%$, obwohl dieses Verfahren die Regel ist, von der laut Vergaberecht nur in Ausnahmefällen abgewichen werden darf (Lorenz et al., 2009, S. 86). ${ }^{3}$

\footnotetext{
${ }^{3}$ Wie später ebenfalls noch an den relevanten Stellen aufgegriffen wird, basiert diese Arbeit unter anderem auf der VOB/A in der Fassung aus 2016. Mit der VOB/A in der Fassung aus 2019 steht dem öffentlichen Verfahren nach § 3a Abs. 1 VOB/A die beschränkte Ausschreibung mit Teilnahmewettbewerb gleichberechtigt als Regelverfahren zur Verfügung.
} 
Öffentliche Einrichtungen - nationale Vergabeverfahren (Anteil an Gesamtzahl der im Jahr 2008 vergebenen Aufträge; durchschnittliche Prozentangaben als arithmetisches Mittel)

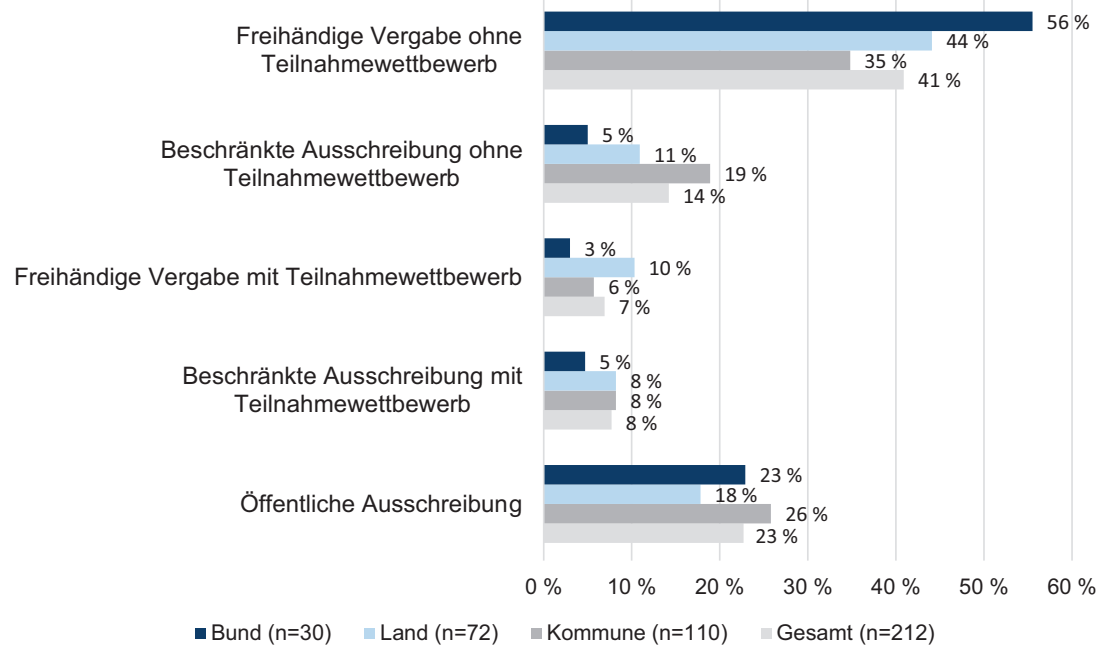

Abbildung 6.5 Anzahl Vergabeverfahren national nach Ebenen (2008) (in Anlehnung an Lorenz et al., 2009, S. 86)

Im Gutachten für die öffentliche Beschaffung attestiert man für Bundesbehörden sogar, dass der Anteil der freihändigen Vergaben im Jahr 2005 88,4 \% betrug (Bundesministerium für Wirtschaft und Technologie, 2007, S. 7).

Die Erhebungen zeigen deutlich, dass für Vergaben unterhalb der Schwellenwerte überwiegend intransparentere Verfahren genutzt werden. Dies müsste im Rahmen einer aktuellen Vergabestatistik (siehe Abschnitt 6.2.2) für den Unterschwellenbereich überprüft werden.

\subsection{Rechtliche Rahmenbedingungen}

Der öffentliche Einkauf wird einerseits von Rechtsbereichen gekennzeichnet, die unmittelbar seine prozessuale Abwicklung zum Gegenstand haben (siehe Abbildung 6.6), andererseits von Rechtsbereichen, die sich an der Schnittstelle zwischen öffentlichem Einkauf und Open Data herausgebildet haben (siehe Abbildung 6.7). In beiden Fällen gibt es nochmal die Unterscheidung zwischen 


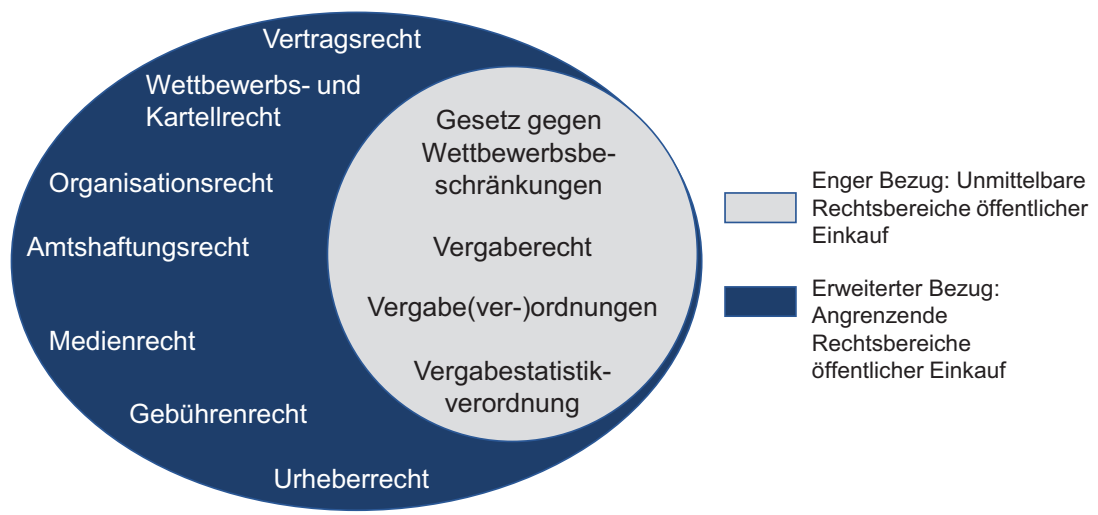

Abbildung 6.6 Rechtsbereiche mit unmittelbarem Einfluss auf den öffentlichen Einkauf

einem engen und einem erweiterten Bezug. Der enge Bezug kennzeichnet die wesentlichen Gesetze, der erweiterte Bezug ergänzende Gesetze.

Bei Rechtsbereichen mit unmittelbarem Einfluss auf den öffentlichen Einkauf besitzen das GWB, das Vergaberecht, die Vergabeverordnungen und die Vergabestatistikverordnung (VergStatVO) einen engen Bezug und werden daher in diesem Kapitel genauer erläutert.

An der Schnittstelle zwischen öffentlichem Einkauf und Open Data sei an dieser Stelle ein Vorgriff auf Abschnitt 7.1 gestattet: Dort werden die wichtigsten Regelungen und somit das Datenschutzgesetz, die Informationsfreiheitsund Transparenzgesetze, das Informationsweiterverwendungsgesetz und die E-Government-Gesetze vertieft. Sie stehen unter dem Vorbehalt fachspezifischer Regelungen und erheben keinen Anspruch auf Vollständigkeit oder an eine Detailanalyse.

Sämtliche zuvor genannten Rechtsbereiche sind im öffentlichen Recht anzusiedeln. Das öffentliche Recht umfasst alle Regelungen zum Verhältnis des einzelnen Bürgers zum Staat und seinen Einrichtungen und das Verhältnis staatlicher Institutionen untereinander (zum Beispiel Bund, Länder, Kommunen).

Das Rechtssystem kann entlang einer Normenhierarchie beschrieben werden (siehe Abbildung 6.8). Zunächst sind das Völkerrecht und das Europarecht zu nennen. Dies sind überstaatliche, aus Prinzipien und Regeln bestehende Rechtsordnungen. Es folgen die Verfassungen des Bundes und der Länder, also Rechtsdokumente, die auf Bundes- oder Landesebene gelten. Anschließend finden Gesetze Anwendung, das heißt Rechtssätze, die vom Träger der Legislative 




Abbildung 6.7 Rechtsbereiche an der Schnittstelle zwischen öffentlichem Einkauf und Open Data

in Form und Verfahren, die in der Verfassung vorgeschrieben sind, erlassen werden. Bundesgesetze sind Rechtsvorschriften, die auf Bundesebene erlassen werden. Die Bundeskompetenzen sind in Art. 73 und Art. 74 Grundgesetz (GG) aufgezählt. Landesgesetze sind Rechtsvorschriften, die auf Landesebene erlassen werden. Die Landeskompetenzen werden nicht einzeln benannt. Nach Art. 70 Abs. 1 GG haben die Länder das Recht der Gesetzgebung, soweit dies nicht per Grundgesetz für den Bund vorgesehen ist. Rechtsverordnungen sind Rechtssätze, die von der Exekutive als Teil eines besonderen Verfahrens erlassen werden. Rechtsnormen regeln das Verhältnis von Menschen zueinander oder zum Staat oder zwischen den Hoheitsträgern. Dies können parlamentarische Gesetze sein, Rechtsverordnungen aufgrund von Gesetzen, öffentlich-rechtliche Satzungen oder unter Umständen sogar Gewohnheitsrecht. Verwaltungsvorschriften (VwV) sind wiederum generell-abstrakte Regelungen oder Anordnungen einer Behörde gegenüber nachgeordneten Behörden. Sie beschränken sich auf den verwaltungsinternen Bereich und sind verbindlich für Behörden und deren Bedienstete aufgrund des Weisungsrechts der übergeordneten Instanz (Bundesministerium des Innern, 2019c; RD Pfeffer, o. A., S. 21 ff.).

In der logischen Reihenfolge der Normenhierarchie wird zunächst der Rechtsraum Europa umrissen, bevor die Umsetzungen in nationales Recht ergänzt werden. Länderspezifische Besonderheiten werden nur dort, wo relevant, näher erläutert. 


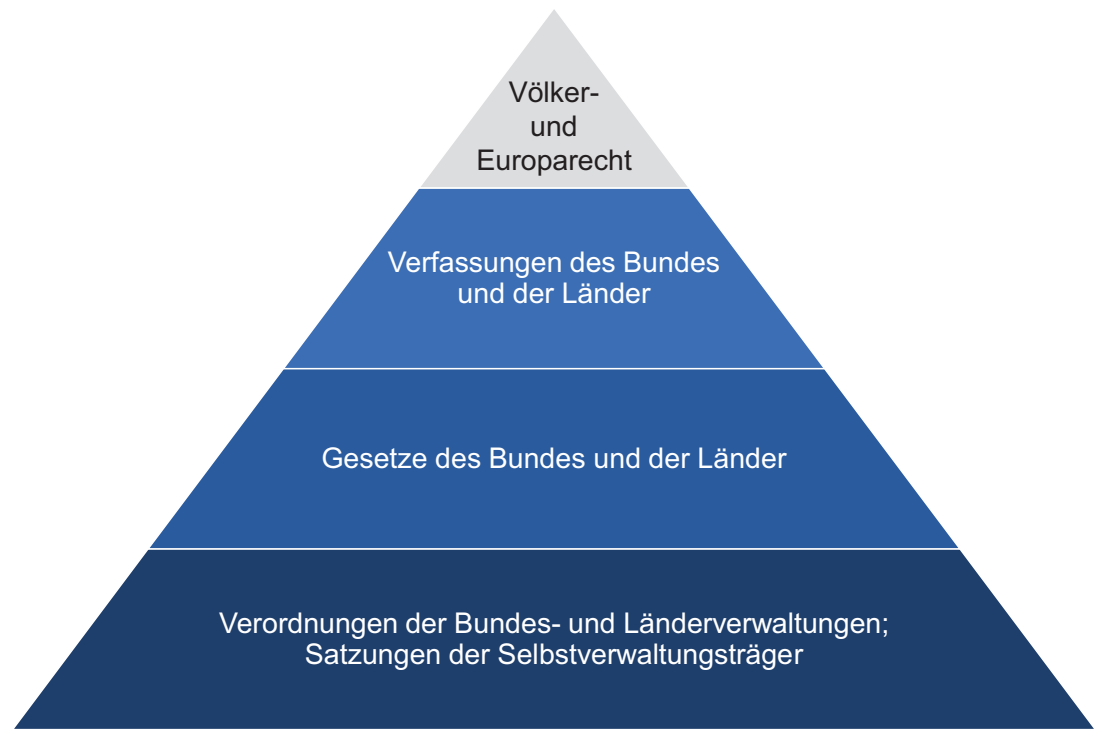

Abbildung 6.8 Normenhierarchie (in Anlehnung an Begerow, 2019)

\subsubsection{Das Vergaberecht}

\subsubsection{Struktur des Vergaberechts}

Als Vergaberecht werden alle Rechtsnormen bezeichnet, die ein öffentlicher Auftraggeber zu beachten hat, wenn er Güter und Leistungen zur Erfüllung seiner Verwaltungsaufgaben beschafft. Das Vergaberecht ist kein Gesetz, sondern es besteht aus einer Vielzahl von Rechtsquellen (siehe Abbildung 6.9).

Wesentlich für das deutsche Vergaberecht ist der vierte Teil des Gesetzes gegen Wettbewerbsbeschränkungen ( $\S 97$ ff. GWB). Dieser gilt sowohl für Vergaben oberhalb als auch unterhalb der Schwellenwerte.

Da diese Arbeit auf die nationalen Vergaben unterhalb der Schwellenwerte fokussiert, sind weiters das Haushaltsgrundsätzegesetz ( $30 \mathrm{HGrG}$ ), die Bundesund Landeshaushaltsordnungen ( $\S 55 \mathrm{BHO}, \mathrm{LHO})$ sowie in fast allen Ländern separate Landesvergabegesetze anzuwenden. Die Länder haben dabei für ihren Bereich nach Art. 30 GG Gesetzgebungskompetenz. Es zeigt sich somit, dass das nationale Vergaberecht im Wesentlichen im Haushaltsrecht begründet ist und damit eher auf Wirtschaftlichkeit und Sparsamkeit ausgerichtet ist (Gentsch, 2012, S. 26 ff.; Rechten \& Röbke, 2017, S. 68). Jedoch hat sich mit dem Ziel 


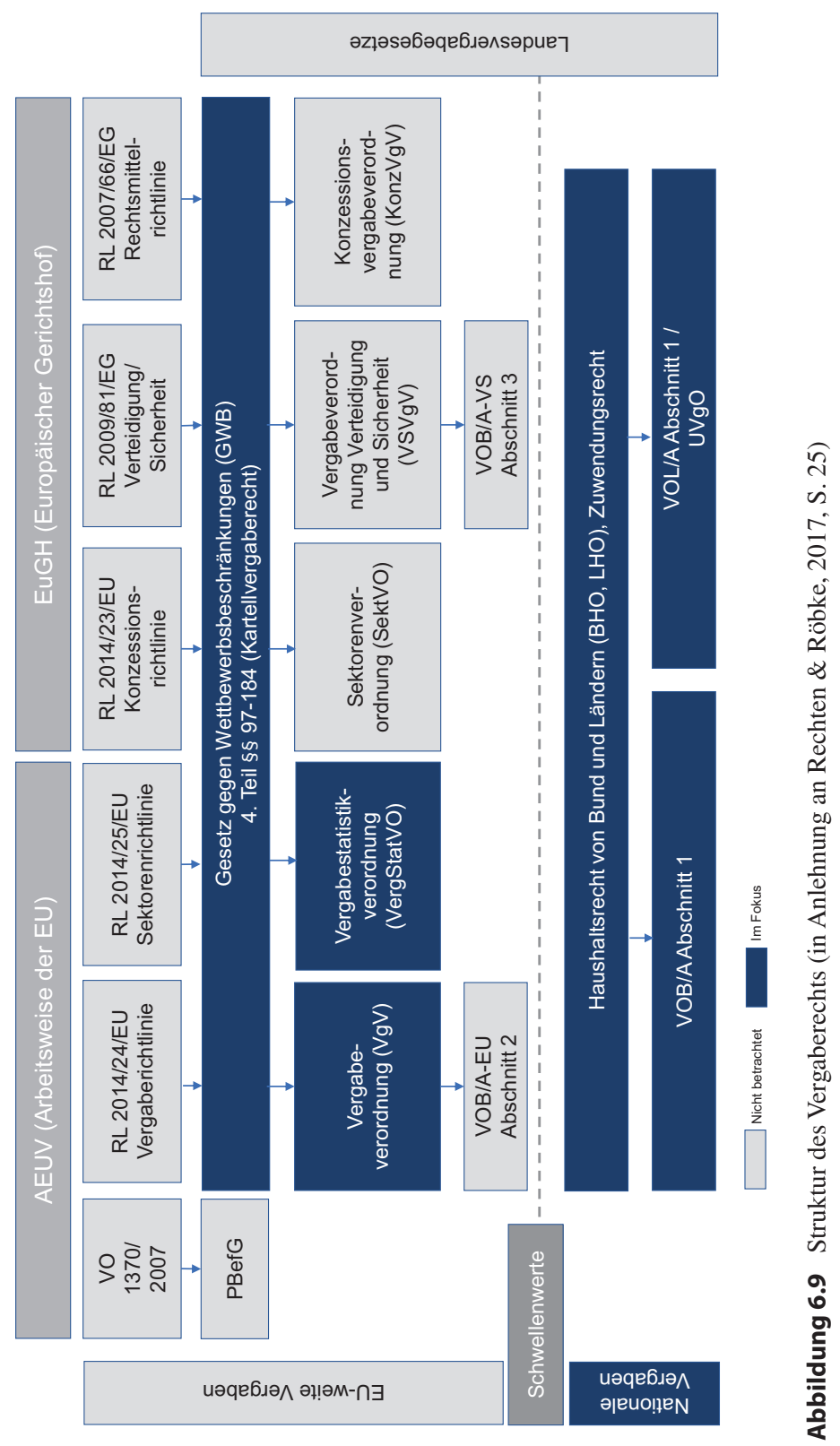


eines europäischen Binnenmarkts die Sichtweise durchaus gewandelt und geht stärker in Richtung Wettbewerb, wobei fraglich ist, wie weitreichend dies in der Praxis gelebt wird (Gentsch, 2012, S. 28).

Auf Verordnungsebene sind neben der Vergabeverordnung (VgV) die VergStatVO zu nennen. Darüber hinaus gilt die Vergabe- und Vertragsordnung für Bauleistungen Teil A. Dies sind allgemeine Bestimmungen für die Vergabe von Bauleistungen (VOB/A 2016 ${ }^{4}$ ). In Abschnitt 1 finden sich die Vorgaben für die Vergaben unterhalb der EU-Schwellenwerte. ${ }^{5}$ Weiters findet die Vergabe- und Vertragsordnung für Leistungen Teil A Anwendung. Dies sind allgemeine Bestimmungen für die Vergabe von Leistungen (VOL/A 20096). In Abschnitt 1 finden sich auch hier die Vorgaben für die Vergaben unterhalb der EU-Schwellenwerte.

In jenen Ländern, wo bereits umgesetzt, gilt die in 2017 auf Bundesebene bekannt gegebene Verfahrensordnung für die Vergabe öffentlicher Liefer- und Dienstleistungsaufträge unterhalb der EU-Schwellenwerte (Unterschwellenvergabeordnung - UvgO). Die UVgO orientiert sich an der bisherigen VOL/A, rückt jedoch stärker an die Regelungen aus der $\mathrm{VgV}$ für die Vergaben im Oberschwellenbereich heran. Sie wird die VOL/A im Zuge der sukzessiven

${ }^{4}$ Die VOB 2016 besteht aus den Teilen A, B und C. VOB/A enthält die Allgemeinen Bestimmungen für die Vergabe von Bauleistungen. Er unterteilt sich in drei weitere Abschnitte: Abschnitt 1 für die Vergabe von Bauleistungen unterhalb der EU-Schwellenwerte (Basisparagrafen), Abschnitt 2 (VOB/A-EU) für Vergabe von Bauleistungen oberhalb der EU-Schwellenwerte und in Abschnitt 3 (VOB/A-VS) für die Vergabe von verteidigungs- und sicherheitsrelevanten Bauleistungen oberhalb der EU-Schwellenwerte. Teil B (VOB/B) enthält die allgemeinen Vertragsbedingungen für die Ausfuhr von Bauleistungen. Teil C (VOB/C) enthält die Allgemeinen Technischen Vertragsbedingungen für die Ausführung von Bauleistungen.

${ }^{5}$ In dieser Arbeit wird noch auf die VOB/A in der Fassung von 2016 Bezug genommen. Dort, wo relevant für diese Arbeit, werden Unterschiede zur neuen Fassung aus 2019 kenntlich gemacht.

${ }^{6}$ Die VOL 2009 besteht aus den Teilen A und B. VOL/A enthält die Allgemeinen Bestimmungen für die Vergabe von Leistungen. Dieser enthält in Abschnitt 1 die Regelungen für die Vergabe von Liefer- und Dienstleistungen unterhalb der EU-Schwellenwerte. Die Regelungen, die oberhalb der EU-Schwellenwerte galten (Abschnitte 2 und 3, sog. EG-Paragrafen), sind nun im 4. Teil des GWB und in der Vergabeverordnung (VgV) enthalten. Verteidigungs- und sicherheitsrelevante Liefer- und Dienstleistungen sind in der VSVgV geregelt. VOL/B enthält die Allgemeinen Vertragsbedingungen für die Ausführung von Leistungen. Die VOL/A Abschnitt 1 wird nach und nach durch die UVgO ersetzt - solange hat sie aber noch Bestand (Rechten \& Röbke, 2017, S. 32 f.). 
Einführung durch Bund, Länder und Kommunen nach und nach ersetzen. ${ }^{7}$ In einigen Ländern wird den Kommunen die Anwendung der UVgO nicht verbindlich vorgeschrieben, sondern nur empfohlen. ${ }^{8}$ Den einzelnen Verwaltungsträgern steht es grundsätzlich frei, die UvGO beziehungsweise VOL/A und VOB/A durch Verwaltungsvorschriften zu konkretisieren (Bundesministerium für Wirtschaft und Energie, 2019c). Landes- oder kommunalspezifische Regelungen werden in dieser Arbeit nur dort, wo relevant, benannt und erörtert.

\subsubsection{Zweck des Vergaberechts}

Der Zweck des Vergaberechts kann in Primär- und Sekundärzwecke unterteilt werden. Primärzwecke ergeben sich unmittelbar aus dem Beschaffungsvorgang und den dazugehörigen Rechten, während der öffentliche Einkauf bei Sekundärzwecken eher unterstützend tätig ist. Sekundärzwecke sind oftmals politisch oder gesellschaftlich motiviert und werden oft noch als vergabefremde Ziele bezeichnet.

Da es sich bei den eingesetzten Haushaltsmitteln von Bund, Ländern und Kommunen um Steuergelder der Bürger handelt, leitet sich als erster Primärzweck Wirtschaftlichkeit ab. Das Haushaltsrecht tendiert somit zu einem Preiswettbewerb, bei welchem ausschließlich der niedrigste Preis entscheidet (Prinzip der Sparsamkeit beziehungsweise Erzielung eines bestimmten Ergebnisses mit geringem Mitteleinsatz). Das Vergaberecht zieht neben dem wirtschaftlichsten Angebot qualitative Faktoren in Betracht (siehe § 127 Abs. 1 GWB) und orientiert sich somit eher an einem Preis-Leistungswettbewerb

\footnotetext{
${ }^{7}$ Dies erfolgt durch Aufnahme eines Anwendungsbefehls zum Inkrafttreten durch Änderungen in den Verwaltungsvorschriften zu $\S 55$ BHO/LHO, Regelungen in den Landesvergabegesetzen sowie auf kommunaler Ebene. Vergabe- und Vertragsordnungen sowie auch die UvGO werden nicht durch den Gesetzgeber erarbeitet, sondern durch privatrechtliche Zusammenschlüsse. Sie besitzen damit keine Rechtsnormqualität. Damit sie eine rechtliche Geltung erlangen, müssen sie durch Einführungserlasse oder Verwaltungsvorschriften der Länder beziehungsweise Kommunen eingeführt werden. Sie besitzen den Charakter einer Verwaltungsvorschrift (Gentsch, 2012, S. 27; Rechten \& Röbke, 2017, S. 33).

${ }^{8}$ Die UVgO wurde bereits eingeführt beim Bund, Baden-Württemberg, Bayern, Berlin, Brandenburg, Bremen, Hamburg, Mecklenburg-Vorpommern, Niedersachsen, NRW, Saarland, Schleswig-Holstein und Thüringen. Bis zur Einführung der UVgO gilt in den anderen Ländern noch die VOL/A Abschnitt 1 (Wagner, 2018).
} 
(Prinzip der Ergiebigkeit beziehungsweise Erzielung des bestmöglichen Ergebnisses mit einem bestimmten Mitteleinsatz) (Eßig, 2008, S. 302).

$\S 97$ Abs. 1 und 2 GWB erläutert weiter:

„Öffentliche Aufträge und Konzessionen werden im Wettbewerb und im Wege transparenter Verfahren vergeben. Dabei werden die Grundsätze der Wirtschaftlichkeit und Verhältnismäßigkeit gewahrt. Die Teilnehmer an einem Vergabeverfahren sind gleich zu behandeln, es sei denn, eine Benachteiligung ist auf Grund dieses Gesetzes ausdrücklich geboten oder gestattet.“

Hier werden neben Wirtschaftlichkeit die Grundprinzipien Wettbewerb, Transparenz, Gleichbehandlung, Nichtdiskriminierung, Wirtschaftlichkeit und Verhältnismäßigkeit genannt. Weitere Grundsätze umfassen die Berücksichtigung des subjektiven Bieterrechts sowie die Verwendung elektronischer Mittel.

Ohne Wettbewerb lässt sich schwerlich eine wirtschaftliche Beschaffung erreichen. Der Primärzweck des Wettbewerbs findet sich nicht nur im EU-Vergaberecht (Oberschwellenbereich), sondern auch im Haushaltsrecht. Er gilt somit auch für den Unterschwellenbereich. ${ }^{9}$ Dabei kann Wettbewerb durch eine Vielzahl von Maßnahmen unterstützt werden, zum Beispiel durch die Wahl der Verfahrensart und damit die Nutzung der öffentlichen Ausschreibung, das Verbot von Wettbewerbsbeschränkungen oder auch die Nutzung der elektronischen Vergabe, um so den Zugang zu Ausschreibungen für Unternehmen zu vereinfachen. Transparenz als weiterer Grundsatz ist ebenfalls eine Voraussetzung, um Wettbewerb zu ermöglichen und zu verstärken. So wird vom Auftraggeber verlangt, die Bieter umfangreich zu informieren, um so eine hohe Beteiligung sicherzustellen und ein nachvollziehbares Vergabeverfahren durchzuführen. Gleichwohl muss dies mit zu schützenden Betriebs- und Geschäftsgeheimnissen einhergehen und kann an dieser Stelle zu Kollisionen führen. Gleichzeitig dient der Transparenzgrundsatz der Vermeidung von Missbrauch öffentlicher Gelder sowie Korruption (Gentsch, 2012, S. 29 f.).

Unter die Sekundärzwecke oder vergabefremde Ziele der öffentlichen Beschaffung fallen zum Beispiel die Berücksichtigung ökologischer Aspekte wie Nachhaltigkeit, sozialpolitischer Zwecke wie die Einhaltung von Mindestlöhnen

${ }^{9}$ Zum Beispiel $§ 2$ Abs. 1 Nr. 2 VOB/A, $§ 2$ Abs. 2 VOL/A sowie $§ 2$ Abs. 1 UVgO. 
oder wirtschaftspolitischer Aspekte wie zum Beispiel die Förderung des Mittelstands oder von Innovationen (Eßig, 2008, S. 301 f.; Rechten \& Röbke, 2017, S. $37 \mathrm{ff}$.).

\subsubsection{Typologie der Auftraggeber}

Der Begriff des Auftraggebers gemäß § 98 GWB umfasst den Einkauf von Waren, Bau- und Dienstleistungen durch öffentliche Auftraggeber (§ 99 GWB), Sektorenauftraggeber ( $§ 100 \mathrm{GWB})$ und Konzessionsgeber ( $\$ 101 \mathrm{GWB})$.

Öffentliche Auftraggeber sind gemäß § 99 Nr. 1 GWB Gebietskörperschaften (zum Beispiel Bund, Länder, Städte, Gemeinden, Landkreise, Regierungsbezirke, Landesdirektionen) sowie deren Sondervermögen (zum Beispiel Eigenbetriebe von Gebietskörperschaften). Weiters umfassen sie funktionale Auftraggeber gemäß § 99 Nr. 2 GWB - dies sind staatsnahe juristische Personen des öffentlichen oder privaten Rechts, die Aufgaben nichtgewerblicher Art im Interesse der Allgemeinheit wahrnehmen und eine besondere Staatsnähe aufweisen, zum Beispiel Stiftungen oder Umweltschutzverbände. Ebenfalls dazu zählen Verbände gemäß $§ 99$ Nr. 3 GWB und auch die Zuwendungsempfänger gemäß §99 Nr.4 GWB, somit natürliche oder juristische Personen des öffentlichen oder privaten Rechts, die Bauvorhaben, die zu mehr als $50 \%$ aus öffentlichen Mitteln finanziert sind, umsetzen.

Sektorenauftraggeber sind dabei Auftraggeber aus den Bereichen Wasser, Elektrizität, Gas und Wärme, Verkehrsleistungen, Häfen und Flughäfen sowie fossilen Brennstoffen, die entweder öffentlich oder unter bestimmten Voraussetzungen auch privat sind. Konzessionsgeber sind entweder öffentliche Auftraggeber oder Sektorenauftraggeber, die Konzessionen vergeben. Private Auftraggeber müssen kein Vergaberecht anwenden.

Im Unterschwellenbereich gilt: Nationales Vergaberecht ist Haushaltsrecht. Hier sind nur die juristischen Personen zur Anwendung des Vergaberechts verpflichtet, die an die Regelungen des BHO und LHO gebunden sind, somit gilt kein funktionaler, sondern ein institutioneller Begriff des Auftraggebers. Dieser beinhaltet nur den Staat und seine Untergliederungen ( $\$ 48$ Abs. 1 HGrG). Juristische Personen des Privatrechts sind also vom Vergaberecht ausgenommen, es sei denn, landesrechtliche Anordnungen oder Zuwendungsbescheide erfordern dies (Rechten \& Röbke, 2017, S. 59 ff.). 


\subsubsection{Vergaberechtliche Definition des öffentlichen Auftrags}

Öffentliche Aufträge sind nach $§ 103$ Abs. 1 GWB

„,entgeltliche Verträge zwischen öffentlichen Auftraggebern und einem oder mehreren Unternehmen über die Beschaffung von Leistungen, die die Lieferung von Waren, die Ausführung von Bauleistungen oder die Erbringung von Dienstleistungen zum Gegenstand haben.“

Da Waren und Dienstleistungen am Markt beschafft werden, ist der Beschaffungszweck ein unverzichtbares Wesenselement des öffentlichen Auftrags.

Davon abzugrenzen sind zum Beispiel Konzessionen. Dabei handelt es sich gemäß $\S 105$ Abs. 1 GWB um entgeltliche Verträge, bei denen die Gegenleistungen zum Beispiel in einem Nutzungs- oder Verwertungsrecht bestehen oder in diesem Recht ergänzt um eine Zahlung. Entgelt bedeutet also nicht nur eine finanzielle Vergütung, sondern jede Art von geldwertem Vorteil.

Weder Schenkungsverträge noch Zuwendungen sind öffentliche Aufträge. Ein Zuwendungsgeber beschafft keine Leistungen, sondern gewährt eine freiwillige Leistung zur Erfüllung eines bestimmten Zweckes (Rechten \& Röbke, 2017, S. $71 \mathrm{ff}$.). Leistungen, die ihren Rechtsgrund in Gesetzen oder Verordnungen haben, stellen ebenfalls keinen öffentlichen Auftrag dar. ${ }^{10}$

\subsubsection{Die Verfahrensarten}

Verfahrensarten sind, wie Abbildung 6.10 illustriert, die öffentliche Ausschreibung, die beschränkte Ausschreibung mit Teilnahmewettbewerb, die Verhandlungsvergabe mit Teilnahmewettbewerb, die beschränkte Ausschreibung ohne Teilnahmewettbewerb, die Verhandlungsvergabe/freihändige Vergabe mit und ohne Teilnahmewettbewerb. Nach Rechtsquellen geordnet stellt sich die Logik wie in Tabelle 6.5 dar.

\footnotetext{
${ }^{10}$ Weitere Ausnahmen finden sich in den $§ \S 107-109,116$ f., 137-140, 149 f. GWB.
} 


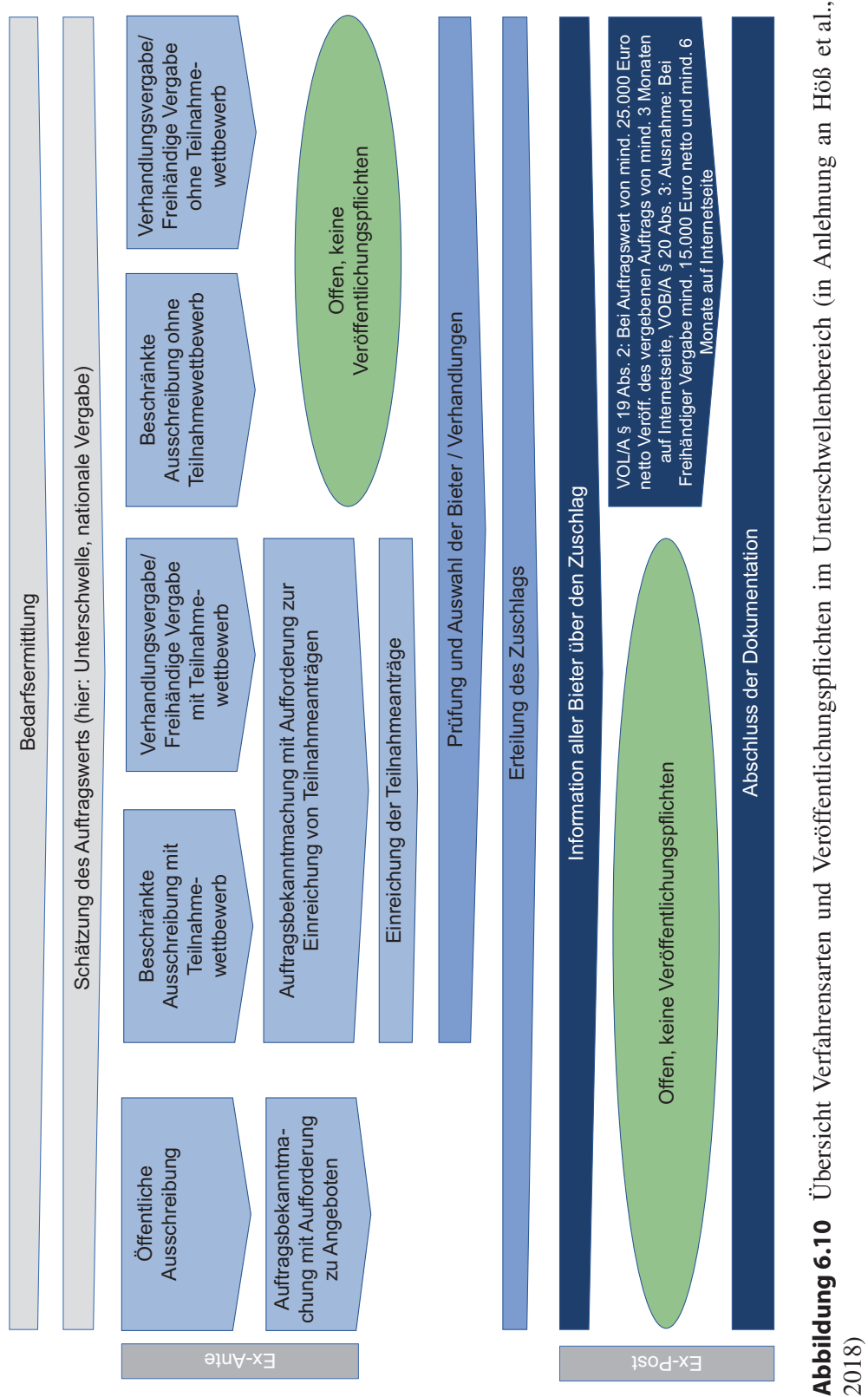


Tabelle 6.5 Übersicht der Verfahrensarten im Unterschwellenbereich

\begin{tabular}{l|l|l|l}
\hline Nr. & VOL/A & UvGO & VOB/A \\
\hline 1 & \multicolumn{2}{|l}{ Öffentliche Ausschreibung } & \\
\hline 2 & \multicolumn{2}{|l|}{ Beschränkte Ausschreibung mit Teilnahmewettbewerb } \\
\hline 3 & \multicolumn{2}{|l|}{ Beschränkte Ausschreibung ohne Teilnahmewettbewerb } \\
\hline 4 & $\begin{array}{l}\text { Freihändige Vergabe mit } \\
\text { oder ohne Teilnahmewett- } \\
\text { bewerb }\end{array}$ & $\begin{array}{l}\text { Verhandlungsvergabe mit } \\
\text { oder ohne Teilnahmewett- } \\
\text { bewerb }\end{array}$ & $\begin{array}{l}\text { Freihändige Vergabe mit } \\
\text { oder ohne Teilnahmewett- } \\
\text { bewerb }\end{array}$ \\
\hline
\end{tabular}

Die öffentliche Ausschreibung (1) entspricht zusammen mit der beschränkten Ausschreibung mit Teilnahmewettbewerb (2) dem Regelverfahren. Dies folgt aus den haushaltsrechtlichen Vorgaben des $\S 30 \mathrm{HGrG}$, den Haushaltsordnungen des Bundes und der Länder ( $\S 55$ BHO, LHO) sowie den Gemeindehaushaltsordnungen. ${ }^{11}$ Im Vergleich $\mathrm{zu}$ den in Abschnitt 6.1.3 aufgeführten Zahlen und Fakten scheinen die Regelverfahren in der Praxis jedoch eher die Ausnahme darzustellen.

Die beschränkte Ausschreibung ohne Teilnahmewettbewerb (3) ist kein Regelverfahren. Sie darf nur unter bestimmten Voraussetzungen genutzt werden, nämlich dann, wenn zuvor eine öffentliche Ausschreibung oder eine beschränkte Ausschreibung mit Teilnahmewettbewerb aufgehoben wurde und die ursprünglichen Auftragsbedingungen nicht entscheidend geändert werden beziehungsweise die öffentliche Ausschreibung oder eine beschränkte Ausschreibung mit Teilnahmewettbewerb nur mit unverhältnismäßigem Aufwand möglich wäre ( 8 Abs. 2 UVgO; § 3a Abs. 2 VOB/A).

Bei der freihändigen Vergabe laut VOB/A und VOL/A können Aufträge mit oder ohne Teilnahmewettbewerb vergeben werden. Ohne Teilnahmewettbewerb bedeutet, dass sich der Auftraggeber direkt an ein oder mehrere Unternehmen wendet und die Konditionen des Auftrags unmittelbar verhandelt (zum Beispiel $\S 3$ Abs. $3 \mathrm{VOB} / \mathrm{A}$ oder $\S 3$ Abs. 1 Satz $3 \mathrm{VOL} / \mathrm{A})$. Mit Einführung der UVgO wird die Verhandlungsvergabe anstelle der freihändigen Vergabe eingeführt ( 8 Abs. 4 UVgO). Nach $\S 12$ Abs. $1 \mathrm{UVgO}$ kann diese auch mit oder ohne Teilnahmewettbewerb durchgeführt werden. Bei Verzicht auf den Wettbewerb schreibt aber $\S 12$ Abs. 2 UVgO dem Auftraggeber vor, mindestens drei Unternehmen zur Abgabe eines Angebots oder zur Teilnahme an Verhandlungen aufzufordern. Doch gibt es auch hier zahlreiche Ausnahmetatbestände. ${ }^{12}$

\footnotetext{
${ }^{11}$ Zum Beispiel § 25 GemHVO NRW.

${ }^{12}$ Nachzulesen in $\S 3$ Abs. 2 Satz 2 VOL/A und $\S 3$ a Abs. 3 VOB/A und $\S 8$ Abs. 4 Nr. 9-14 $\mathrm{UVgO}$.
} 


\subsubsection{Schwellenwerte}

Die aktuellen Schwellenwerte sind in Tabelle 6.6 aufgeführt ${ }^{13}$. Unterhalb dieser Grenzen gelten die Regelungen für den Unterschwellenbereich, darüber jene für den Oberschwellenbereich gemäß $\S 106$ GWB (Bundesministerium für Wirtschaft und Energie, 2019c).

Tabelle 6.6 Wertgrenzen Vergaberecht in ihrer geltenden Fassung bis 31.12.2019 (in Anlehnung an Bundesministerium für Wirtschaft und Energie, 2019c)

\begin{tabular}{|c|c|c|}
\hline Typ & Schwellenwerte in $€$ & Rechtsquelle \\
\hline Bauaufträge und Konzessionsvergabe & $\begin{array}{l}\text { 5.548.000 zuzüglich } \\
\text { Mehrwertsteuer }\end{array}$ & \multirow[t]{2}{*}{$\begin{array}{l}\text { Delegierte Verordnung } \\
\text { (EU) 2017/2364 }\end{array}$} \\
\hline $\begin{array}{l}\text { Liefer- und Dienstleistungsaufträge } \\
\text { von Sektorenauftraggebern }\end{array}$ & $\begin{array}{l}443.000 \text { zuzüglich } \\
\text { Mehrwertsteuer }\end{array}$ & \\
\hline Konzessionen & $\begin{array}{l}\text { 5.548.000 zuzüglich } \\
\text { Mehrwertsteuer }\end{array}$ & $\begin{array}{l}\text { Delegierte Verordnung } \\
\text { (EU) 2017/2366 }\end{array}$ \\
\hline $\begin{array}{l}\text { Verteidigungs- und sicherheits- } \\
\text { relevante Liefer- und Dienstleistungs- } \\
\text { aufträge }\end{array}$ & $\begin{array}{l}443.000 \text { zuzüglich } \\
\text { Mehrwertsteuer }\end{array}$ & \multirow[t]{2}{*}{$\begin{array}{l}\text { Verordnung (EU) } \\
2017 / 2367\end{array}$} \\
\hline $\begin{array}{l}\text { Verteidigungs- und sicherheits- } \\
\text { relevante Bauaufträge }\end{array}$ & $\begin{array}{l}\text { 5.548.000 zuzüglich } \\
\text { Mehrwertsteuer }\end{array}$ & \\
\hline $\begin{array}{l}\text { Liefer- und Dienstleistungsaufträge } \\
\text { für oberste, obere Bundesbehörden } \\
\text { und vergleichbare Bundesein- } \\
\text { richtungen }\end{array}$ & $\begin{array}{l}\text { 144.000 zuzüglich } \\
\text { Mehrwertsteuer }\end{array}$ & \multirow[t]{2}{*}{$\begin{array}{l}\text { Delegierte Verordnung } \\
\text { (EU) 2017/2365 }\end{array}$} \\
\hline Liefer- und Dienstleistungen & $\begin{array}{l}221.000 \text { zuzüglich } \\
\text { Mehrwertsteuer }\end{array}$ & \\
\hline $\begin{array}{l}\text { Vergabe von Sozialen und anderen } \\
\text { Besonderen Dienstleistungen }\end{array}$ & $\begin{array}{l}750.000 \text { zuzüglich } \\
\text { Mehrwertsteuer }\end{array}$ & Richtlinie 2014/24/EU \\
\hline
\end{tabular}

Unterhalb dieser Grenzen legen die Länder nochmals landesspezifische Minimumwerte fest, bei deren Unterschreitung direkt beschafft werden kann. Sie liegen für Liefer- und Dienstleistungsaufträge sowie Bauaufträge bei 10.000 bis 100.000 Euro pro Land (Brockhoff et al., 2019, S. 50 ff.). Demnach besteht hier nochmals ein großer Spielraum für direkte Vergaben ohne Kenntnisnahme durch die Öffentlichkeit.

\footnotetext{
${ }^{13}$ Eine Anpassung dieser Schwellenwerte ist zum 01. Januar 2020 erfolgt (Cosinex GmbH, 2019). Diese Arbeit referenziert bei Veröffentlichung noch auf die bis 31.12.2019 gültigen Werte.
} 


\subsubsection{Pflichten zur Transparenz im Vergaberecht}

Bei öffentlichen Aufträgen besteht eine Transparenzpflicht gegenüber der Öffentlichkeit, die durch die Bekanntgabe der Ausschreibung oder des Teilnahmewettbewerbs und die Publikation vergebener Aufträge erfüllt wird. Ex-ante bedeutet somit die Ankündigung eines Ausschreibungsverfahrens über die Auftragsbekanntmachung (ABM), ex-post bedeutet die Information nach erfolgter Vergabe über die Vergabebekanntmachung (VBM). Tabelle 6.7 stellt dies dar.

Tabelle 6.7 Kurzübersicht der Transparenzpflichten für Bekanntmachungen im Ober- und Unterschwellenbereich

\begin{tabular}{|c|c|c|c|}
\hline $\begin{array}{l}\text { Art der Bekannt- } \\
\text { machung }\end{array}$ & Typ & $\begin{array}{l}\text { Transparenz- } \\
\text { pflicht OSB } \\
\text { (= EU-weit) }\end{array}$ & $\begin{array}{l}\text { Transparenzpflicht USB } \\
\text { (= National) }\end{array}$ \\
\hline Vorinformation & Ex-ante & $\mathrm{Ja}$ & Nein, nicht vorgesehen \\
\hline $\begin{array}{l}\text { Auftragsbekannt- } \\
\text { machung (ABM) }\end{array}$ & Ex-ante & $\mathrm{Ja}$ & $\begin{array}{l}\text { Ja, bei öffentlicher Ausschreibung, } \\
\text { beschränkter Ausschreibung mit Teil- } \\
\text { nahmewettbewerb, freihändiger Vergabe } \\
\text { und Verhandlungsvergabe mit Teil- } \\
\text { nahmewettbewerb }\end{array}$ \\
\hline $\begin{array}{l}\text { Vergabebekannt- } \\
\text { machung (VBM) }\end{array}$ & Ex-post & $\mathrm{Ja}$ & $\begin{array}{l}\text { Ja, bei beschränkten Ausschreibungen } \\
\text { ohne Teilnahmewettbewerb und Ver- } \\
\text { handlungsvergaben ohne Teilnahme- } \\
\text { wettbewerb informiert der Auftraggeber } \\
\text { gemäß } \S 30 \text { UVgO beziehungsweise } \\
\S 19 \text { Abs. } 2 \text { VOL/A über jeden ver- } \\
\text { gebenen Auftrag ab einem Auftragswert } \\
\text { von } 25.000 \text { Euro netto beziehungsweise } \\
\text { 15.000 Euro netto bei freihändigen } \\
\text { Verfahren laut } \S 20 \text { Abs. } 3 \text { VOB/A (Höß } \\
\text { et al., 2018, S. 33) }\end{array}$ \\
\hline $\begin{array}{l}\text { Bekanntmachung } \\
\text { von Auftrags- } \\
\text { änderungen }\end{array}$ & Ex-post & $\mathrm{Ja}$ & Nein, nicht vorgesehen \\
\hline
\end{tabular}

Es wird deutlich, dass die Anforderungen an Information und Transparenz bezogen auf den Oberschwellenbereich höher sind als in Bezug auf den Unterschwellenbereich. Dies irritiert vor dem Hintergrund, dass Ausschreibungen und Vergaben im Oberschwellenbereich sowohl nach Volumen als auch nach Anzahl den weitaus geringeren Anteil einnehmen (siehe Abschnitt 6.1). 
In den folgenden Ausführungen stehen insbesondere die Auftrags- und die Vergabebekanntmachung im Fokus, da diese für den Unterschwellenbereich im heutigen Vergabeprozess die einzigen verpflichtenden Bekanntgaben gegenüber der Öffentlichkeit darstellen. Somit haben sie eine große Bedeutung sowohl für Transparenz als auch für Beteiligungsmöglichkeiten und letztlich für die Wirtschaftspolitik insgesamt.

\subsubsection{Nationale Auftragsbekanntmachung}

Wenn öffentliche Auftraggeber einen öffentlichen Auftrag vergeben wollen, müssen sie dies gemäß Transparenzgrundsatz öffentlich anzeigen ( $\$ 37$ Abs. 1 GWB, § 12 Abs. 1 VOB/A, § 12 VOL/A und § 28 UvGO). Die Auftragsbekanntmachung im Sinne der ex-ante Veröffentlichung ist der Auftakt des formellen Vergabeverfahrens. Nur mit dieser Information ist es Bewerbern und Bietern möglich, eine Entscheidung für eine Teilnahme oder Nichtteilnahme zu treffen. Daher besitzt die Auftragsbekanntmachung einen hohen Stellenwert und der öffentliche Auftraggeber muss diese aus der Sicht eines möglichen Bewerbers fertigen (Rechten \& Röbke, 2017, S. 154 f.).

Bei der Bekanntmachung wird zwischen Vergaben der Oberschwelle und Unterschwelle unterschieden. Vergabeverfahren im Oberschwellenbereich sind europaweit mithilfe von vorgeschriebenen Standardformularen gemäß Verordnung (EU) Nr. 2015/1986 auszuschreiben und im Supplement zum Amtsblatt (ABl.) der EU bekannt zu geben, welches ausschließlich elektronisch herausgegeben wird. Auf der Seite https://ted.europa.eu können interessierte Bewerber Einblick in alle aktuellen Bekanntmachungen nehmen (Rechten \& Röbke, 2017, S. 154 f.).

Im Unterschwellenbereich muss die Bekanntmachung nur auf nationaler Ebene erfolgen und die Auflagen für die Gestaltung sind für öffentliche Auftraggeber geringer. So sind zum Beispiel keine EU-weiten Bekanntmachungen oder die Verwendung von Standardformularen vorgeschrieben (Rechten \& Röbke, 2017, S. 157).

Seit Bekanntgabe der UvGO Bund für Liefer- und Dienstleistungen im Jahr 2017 ist es Pflicht, Auftragsbekanntmachungen auf den Internetseiten des Auftraggebers oder Internetportalen zu veröffentlichen. Zusätzlich - und nun sekundär - können Auftragsbekanntmachungen in den üblichen Printmedien (zum Beispiel Tageszeitungen, amtlichen Veröffentlichungsblättern) veröffentlicht werden. Auftragsbekanntmachungen im Internet müssen zentral über die Suchfunktion des Portals https://www.service.bund.de/ ermittelt werden können. Im Unterschied dazu fordern $\S 12$ Abs. 1 Nr. 2 VOB/A und $\S 12$ Abs. 2 VOL/A die Veröffentlichung der Bekanntmachung in Printmedien vor der Veröffentlichung auf Internetportalen. Hier wird das Internet demnach noch als bloße Sekundärquelle genannt. 
UVgO, VOL/A und VOB/A listen die Angaben auf, die je nach Auftragsgegenstand mindestens in die Bekanntmachung aufgenommen werden sollen (siehe Tabelle 6.8). UvGO $\S 28$ Abs. 2 entspricht im Wesentlichen $\S 12$ Abs. 2 VOL/A. Ähnliche oder gleiche Inhalte sind in derselben Zeile gegenübergestellt.

Tabelle 6.8 Rechtliche Pflichtangaben zur ex-ante Auftragsbekanntmachung im Unterschwellenbereich nach $\S 12 \mathrm{VOB} / \mathrm{A}, \S 12 \mathrm{VOL} / \mathrm{A}$ und $\S 28 \mathrm{UvGO}$ (das Kürzel ,./.“ bedeutet, dass es hierzu keine Angaben in der Quelle gibt)

\begin{tabular}{|c|c|c|}
\hline VOB/A $§ 12$ Abs. 2 & VOL/A $\$ 12$ Abs. 2 & UvGO $§ 28$ Abs. 2 \\
\hline $\begin{array}{l}\text { Name, Anschrift, Tele- } \\
\text { fon-, Faxnummer sowie } \\
\text { E-Mailadresse des Auftrag- } \\
\text { gebers (Vergabestelle) }\end{array}$ & $\begin{array}{l}\text { Bezeichnung und die } \\
\text { Anschrift der zur Angebots- } \\
\text { abgabe auffordernden Stelle, } \\
\text { der den Zuschlag erteilenden } \\
\text { Stelle sowie der Stelle, bei } \\
\text { der die Angebote oder Teil- } \\
\text { nahmeanträge einzureichen } \\
\text { sind }\end{array}$ & $\begin{array}{l}\text { Bezeichnung und } \\
\text { die Anschrift der zur } \\
\text { Angebotsabgabe auf- } \\
\text { fordernden Stelle, der den } \\
\text { Zuschlag erteilenden } \\
\text { Stelle sowie der Stelle, } \\
\text { bei der die Angebote oder } \\
\text { Teilnahmeanträge einzu- } \\
\text { reichen sind }\end{array}$ \\
\hline Gewähltes Vergabeverfahren & Art der Vergabe & Verfahrensart \\
\hline $\begin{array}{l}\text { Gegebenenfalls Auftrags- } \\
\text { vergabe auf elektronischem } \\
\text { Wege und Verfahren der } \\
\text { Ver- und Entschlüsselung }\end{array}$ & $\begin{array}{l}\text { Form, in der die Teilnahme- } \\
\text { anträge oder Angebote einzu- } \\
\text { reichen sind }\end{array}$ & $\begin{array}{l}\text { Form, in der die Teilnahme- } \\
\text { anträge oder Angebote } \\
\text { einzureichen sind }\end{array}$ \\
\hline Art des Auftrags &.$/$ &. \\
\hline Ort der Ausführung & Ort der Leistungserbringung & Ort der Leistungserbringung \\
\hline $\begin{array}{l}\text { Art und Umfang der } \\
\text { Leistung }\end{array}$ & Art und Umfang der Leistung & $\begin{array}{l}\text { Art und Umfang der } \\
\text { Leistung }\end{array}$ \\
\hline. & $\begin{array}{l}\text { Gegebenenfalls die Anzahl, } \\
\text { Größe und Art der einzelnen } \\
\text { Lose }\end{array}$ & $\begin{array}{l}\text { Gegebenenfalls die Anzahl, } \\
\text { Größe und Art der einzelnen } \\
\text { Lose }\end{array}$ \\
\hline $\begin{array}{l}\text { Angaben über den Zweck } \\
\text { der baulichen Anlage oder } \\
\text { des Auftrags, wenn auch } \\
\text { Planungsleistungen } \\
\text { gefordert werden }\end{array}$ &.$/$ &.$/$ \\
\hline $\begin{array}{l}\text { Falls die bauliche Anlage } \\
\text { oder der Auftrag in mehrere } \\
\text { Lose aufgeteilt ist, Art und } \\
\text { Umfang der einzelnen Lose } \\
\text { und Möglichkeit, Angebote } \\
\text { für eines, mehrere } \\
\text { oder alle Lose einzureichen }\end{array}$ &.$/$ &.$/$ \\
\hline
\end{tabular}


Tabelle 6.8 (Fortsetzung)

\begin{tabular}{|c|c|c|}
\hline VOB/A $\$ 12$ Abs. 2 & VOL/A $§ 12$ Abs. 2 & UvGO $\$ 28$ Abs. 2 \\
\hline $\begin{array}{l}\text { Zeitpunkt, bis zu dem die } \\
\text { Bauleistung beendet werden } \\
\text { soll oder die Dauer des Bau- } \\
\text { leistungsauftrages; sofern } \\
\text { möglich, Zeitpunkt, zu dem } \\
\text { die Bauleistung begonnen } \\
\text { werden muss }\end{array}$ &.$/$ &.$/$ \\
\hline $\begin{array}{l}\text { Gegebenenfalls Angaben } \\
\text { nach } \$ 8 \text { Abs. } 2 \text { Nr. } 3 \\
\text { VOB/A zur Zulässigkeit von } \\
\text { Nebenangeboten }\end{array}$ & $\begin{array}{l}\text { Gegebenenfalls } \\
\text { die Zulassung von } \\
\text { Nebenangeboten }\end{array}$ & $\begin{array}{l}\text { Gegebenenfalls die } \\
\text { Zulassung von Neben- } \\
\text { angeboten }\end{array}$ \\
\hline $\begin{array}{l}\text { Name und Anschrift, } \\
\text { Telefon- und Faxnummer, } \\
\text { E-Mailadresse der Stelle, bei } \\
\text { der die Vergabeunterlagen } \\
\text { und zusätzliche Unterlagen } \\
\text { angefordert und eingesehen } \\
\text { werden können }\end{array}$ & $\begin{array}{l}\text { Bezeichnung und die } \\
\text { Anschrift der Stelle, die die } \\
\text { Vergabeunterlagen abgibt } \\
\text { oder bei der sie eingesehen } \\
\text { werden können }\end{array}$ & $\begin{array}{l}\text { die elektronische Adresse, } \\
\text { unter der die Vergabe- } \\
\text { unterlagen abgerufen } \\
\text { werden können oder die } \\
\text { Bezeichnung und die } \\
\text { Anschrift der Stelle, die die } \\
\text { Vergabeunterlagen abgibt } \\
\text { oder bei der sie eingesehen } \\
\text { werden können }\end{array}$ \\
\hline $\begin{array}{l}\text { (Bei Teilnahmeantrag:) Frist } \\
\text { für den Eingang der Anträge } \\
\text { auf Teilnahme, Anschrift an } \\
\text { die diese Anträge zu richten } \\
\text { sind, Tag, an dem die Auf- } \\
\text { forderungen zur Angebots- } \\
\text { abgabe spätestens abgesandt } \\
\text { werden }\end{array}$ &.$/$ &.$\%$ \\
\hline $\begin{array}{l}\text { Frist für den Eingang der } \\
\text { Angebote; Bindefrist }\end{array}$ & $\begin{array}{l}\text { Teilnahme- oder Angebots- } \\
\text { und Bindefrist }\end{array}$ & $\begin{array}{l}\text { Teilnahme- oder Angebots- } \\
\text { und Bindefrist }\end{array}$ \\
\hline.$/$. & $\begin{array}{l}\text { Etwaige Bestimmungen über } \\
\text { die Ausführungsfrist }\end{array}$ & $\begin{array}{l}\text { Etwaige Bestimmungen } \\
\text { über die Ausführungsfrist }\end{array}$ \\
\hline $\begin{array}{l}\text { Anschrift, an die die } \\
\text { Angebote zu richten sind, } \\
\text { gegebenenfalls auch } \\
\text { Anschrift, an die Angebote } \\
\text { elektronisch zu übermitteln } \\
\text { sind }\end{array}$ &.$/$ &.$/$ \\
\hline
\end{tabular}


Tabelle 6.8 (Fortsetzung)

\begin{tabular}{|c|c|c|}
\hline VOB/A § 12 Abs. 2 & VOL/A $\$ 12$ Abs. 2 & UvGO $§ 28$ Abs. 2 \\
\hline $\begin{array}{l}\text { Sprache, in der die Angebote } \\
\text { abgefasst sein müssen }\end{array}$ &.$\%$ &. \\
\hline $\begin{array}{l}\text { Datum, Uhrzeit und Ort des } \\
\text { Eröffnungstermins sowie } \\
\text { Angabe, welche Personen bei } \\
\text { der Eröffnung der Angebote } \\
\text { anwesend sein dürfen }\end{array}$ &.$/$ &. \\
\hline $\begin{array}{l}\text { Gegebenenfalls geforderte } \\
\text { Sicherheiten }\end{array}$ & $\begin{array}{l}\text { Die Höhe etwa geforderter } \\
\text { Sicherheitsleistungen }\end{array}$ & $\begin{array}{l}\text { Die Höhe etwa geforderter } \\
\text { Sicherheitsleistungen }\end{array}$ \\
\hline $\begin{array}{l}\text { Wesentliche Finanzierungs- } \\
\text { und Zahlungsbedingungen } \\
\text { und/oder Hinweise auf die } \\
\text { maßgeblichen Vorschriften, } \\
\text { in denen sie enthalten sind }\end{array}$ & $\begin{array}{l}\text { Die wesentlichen Zahlungs- } \\
\text { bedingungen oder Angabe } \\
\text { der Unterlagen, in denen sie } \\
\text { enthalten sind }\end{array}$ & $\begin{array}{l}\text { Die wesentlichen Zahlungs- } \\
\text { bedingungen oder Angabe } \\
\text { der Unterlagen, in denen sie } \\
\text { enthalten sind }\end{array}$ \\
\hline $\begin{array}{l}\text { Gegebenenfalls Rechtsform, } \\
\text { die die Bietergemeinschaft } \\
\text { nach der Auftragsvergabe } \\
\text { haben muss }\end{array}$ &.$\%$ &. \\
\hline $\begin{array}{l}\text { Verlangte Nachweise für die } \\
\text { Beurteilung der Eignung des } \\
\text { Bewerbers oder Bieters }\end{array}$ & $\begin{array}{l}\text { Die mit dem Angebot oder } \\
\text { dem Teilnahmeantrag vor- } \\
\text { zulegenden Unterlagen, die } \\
\text { die Auftraggeber für die } \\
\text { Beurteilung der Eignung } \\
\text { des Bewerbers oder Bieters } \\
\text { verlangen }\end{array}$ & $\begin{array}{l}\text { Die mit dem Angebot oder } \\
\text { dem Teilnahmeantrag vor- } \\
\text { zulegenden Unterlagen, die } \\
\text { der Auftraggeber für die } \\
\text { Beurteilung der Eignung } \\
\text { des Bewerbers oder Bieters } \\
\text { und des Nichtvorliegens von } \\
\text { Ausschlussgründen verlangt }\end{array}$ \\
\hline $\begin{array}{l}\text { Gegebenenfalls Höhe und } \\
\text { Bedingungen für die } \\
\text { Zahlung des Betrages, } \\
\text { der für die Unterlagen zu } \\
\text { entrichten ist }\end{array}$ & $\begin{array}{l}\text { Sofern verlangt, die Höhe } \\
\text { der Kosten für Verviel- } \\
\text { fältigungen der Vergabe- } \\
\text { unterlagen bei Öffentlichen } \\
\text { Ausschreibungen }\end{array}$ &. \\
\hline $\begin{array}{l}\text { Die Zuschlagskriterien, } \\
\text { sofern diese nicht in den } \\
\text { Vergabeunterlagen genannt } \\
\text { werden, und gegebenenfalls } \\
\text { deren Gewichtung }\end{array}$ & $\begin{array}{l}\text { Angabe der Zuschlags- } \\
\text { kriterien, sofern diese nicht } \\
\text { in den Vergabeunterlagen } \\
\text { genannt werden }\end{array}$ & $\begin{array}{l}\text { Angabe der Zuschlags- } \\
\text { kriterien, sofern diese nicht } \\
\text { in den Vergabeunterlagen } \\
\text { genannt werden }\end{array}$ \\
\hline
\end{tabular}

(Fortsetzung) 
Tabelle 6.8 (Fortsetzung)

\begin{tabular}{|c|c|c|}
\hline VOB/A $§ 12$ Abs. 2 & VOL/A $§ 12$ Abs. 2 & UvGO $\$ 28$ Abs. 2 \\
\hline $\begin{array}{l}\text { Name und Anschrift der } \\
\text { Stelle, an die sich der } \\
\text { Bewerber oder Bieter zur } \\
\text { Nachprüfung behaupteter } \\
\text { Verstöße gegen Vergabe- } \\
\text { bestimmungen wenden kann }\end{array}$ &. &.$\%$ \\
\hline. &.$\%$ & $\begin{array}{l}\text { Gegebenenfalls in den } \\
\text { Fällen des } § 29 \text { Abs. } 3 \text { die } \\
\text { Maßnahmen zum Schutz } \\
\text { der Vertraulichkeit und die } \\
\text { Informationen zum Zugriff } \\
\text { auf die Vergabeunterlagen }\end{array}$ \\
\hline
\end{tabular}

\subsubsection{Nationale Vergabebekanntmachung}

Im Unterschwellenbereich erfolgen, wie die statistischen Daten in Abschnitt 6.1 aufgezeigt haben, zum Teil Vergaben ohne vorherige allgemeine Bekanntmachung, also ohne ex-ante Transparenz. Dies betrifft insbesondere Vergaben auf der Grundlage beschränkter Ausschreibungen ohne Teilnahmewettbewerb und freihändiger Vergaben.

Hier sieht das Vergaberecht die Veröffentlichung der vergebenen Aufträge im Nachhinein vor (ex-post Transparenz). Bei beschränkten Ausschreibungen ohne Teilnahmewettbewerb und Verhandlungsvergaben ohne Teilnahmewettbewerb informiert der Auftraggeber gemäß $\S 30 \mathrm{Abs} .1 \mathrm{UVgO}$ oder $\S 19 \mathrm{Abs}$. 2 VOL/A über jeden vergebenen Auftrag ab einem Auftragswert von 25.000 Euro netto für die Dauer von drei Monaten und nach $\S 20 \mathrm{Abs} .3 \mathrm{VOB} / \mathrm{A}$ für die Dauer von sechs Monaten auf seiner Internetseite oder Internetportalen. Für freihändige Vergaben gilt gemäß $\S 20$ Abs. $3 \mathrm{VOB} / \mathrm{A}$ eine Veröffentlichungsfrist von sechs Monaten für Vergaben ab einem Auftragswert von 15.000 Euro netto. Tabelle 6.9 listet die verlangten Mindestangaben in der Vergabebekanntmachung auf.

UvGO $\S 30$ Abs. 2 entspricht im Wesentlichen $\S 19$ Abs. 2 VOL/A. Es wird deutlich, dass die Pflichten zur Bekanntmachung ex-post signifikant geringer sind als ex-ante. Das heißt, die Informationsmöglichkeiten unterscheiden sich erheblich je nach gewählter Verfahrensart beziehungsweise zielen insbesondere auf die Erzeugung von Wettbewerb ab (ex-ante), weniger auf die Transparenz in Bezug auf die Art der Umsetzung des Vertrags (ex-post). So gibt es keine Vergabebekanntmachungen für die Verfahrensarten der öffentlichen Ausschreibung, beschränkten Ausschreibung mit Teilnahmewettbewerb und Verhandlungsvergabe/Freihändigen Vergabe mit Teilnahmewettbewerb. Für diese werden die Vergabeergebnisse nicht öffentlich bekannt gegeben. 
Tabelle 6.9 Rechtliche Pflichtangaben zur ex-post Vergabebekanntmachung im Unterschwellenbereich nach $\S 20 \mathrm{VOB} / \mathrm{A}, \S 19 \mathrm{VOL} / \mathrm{A}$ und $\S 30 \mathrm{UvGO}$

\begin{tabular}{|c|c|c|}
\hline VOB/A $§ 20$ Abs. 3 & VOL/A § 19 Abs. 2 & UvGO $§ 30$ Abs. 1 \\
\hline Auftraggeber & Auftraggeber & $\begin{array}{l}\text { Name und Anschrift } \\
\text { des Auftraggebers und } \\
\text { dessen Beschaffungsstelle }\end{array}$ \\
\hline Auftragsgegenstand & Auftragsgegenstand &.$/$ \\
\hline Vergabeverfahren & Vergabeverfahren & Verfahrensart \\
\hline Art und Umfang der Leistung & Art und Umfang der Leistung & $\begin{array}{l}\text { Art und Umfang der } \\
\text { Leistung }\end{array}$ \\
\hline Ort der Ausführung & Ort der Ausführung &.$/$ \\
\hline $\begin{array}{l}\text { Name des beauftragten } \\
\text { Unternehmens }\end{array}$ & $\begin{array}{l}\text { Name des beauftragten } \\
\text { Unternehmens }\end{array}$ & $\begin{array}{l}\text { Name des beauftragten } \\
\text { Unternehmens; soweit es } \\
\text { sich um eine natürliche } \\
\text { Person handelt, ist deren } \\
\text { Einwilligung einzuholen } \\
\text { oder zu anonymisieren }\end{array}$ \\
\hline. &.$/$. & Zeitraum der Ausführung \\
\hline
\end{tabular}

\subsubsection{Vergaberechtsreform und eVergabe}

Mit der am 18. April 2016 in Kraft getretenen Vergaberechtsreform wurde die Struktur des Vergaberechts für die Vergabe öffentlicher Aufträge oberhalb der EU-Schwellenwerte neugestaltet. Mit dieser Reform werden die drei EU-Vergaberichtlinien aus dem Jahr 2014 in deutsches Recht umgesetzt: Die Richtlinie über die öffentliche Auftragsvergabe (2014/24/EU), die Richtlinie über die Vergabe von Aufträgen in den Bereichen Wasser-, Energie- und Verkehrsversorgung sowie der Postdienste (2014/25/EU) und die Richtlinie über die Vergabe von Konzessionen (2014/23/EU).

Bisher waren die Regelungen zur Vergabe öffentlicher Aufträge im Oberschwellenbereich im Gesetz gegen Wettbewerbsbeschränkungen (GWB) geregelt und die Verfahrensregelungen in der VOB/A, der VOL/A und der VOF. Grundsätzliche gesetzliche Regelungen werden auch weiterhin im GWB getroffen, aber die Verfahrensregelungen im Wesentlichen in der $\mathrm{VgV}$ und für Vergabeverfahren im Bauleistungsbereich ergänzend in der VOB/A. Die VOL/A wird damit oberhalb der EU-Schwellenwerte nicht mehr angewendet. Die VOF entfällt ersatzlos.

Das GWB enthält im vierten Teil „Vergabe von öffentlichen Aufträgen und Konzessionen“ in den $\S \S 97$ ff. Grundlegendes über die Vergabe von öffentlichen Aufträgen und Konzessionen sowie den Rechtsschutz. Der vierte Teil gilt nach 
$\S 106$ GWB für Vergaben, deren prognostizierter Auftrags- oder Vertragswert die jeweiligen Schwellenwerte erreicht oder überschreitet (netto). Der vierte Teil des GWB enthält in $\S 97$ unter anderem auch den Gleichbehandlungs-, den Transparenz- und den Wettbewerbsgrundsatz. Dies sind Grundsätze, die für alle Vergaben Gültigkeit besitzen.

Die Sektorenverordnung (SektVO) und die Verordnung über die Vergabe in den Bereichen Verteidigung und Sicherheit (VSVgV) regeln die Vergaben in diesen besonderen Bereichen.

Gleichzeitig wurde mit der Vergaberechtsreform 2016 festgelegt, dass grundsätzlich die elektronische Datenübermittlung (eVergabe) gilt. Nach einer Übergangszeit ist es verpflichtend, für alle Bekanntmachungen und den Daten- und Dokumentenaustausch auf elektronische Mittel zurückzugreifen. Für den Oberschwellenbereich gilt die Pflicht zur Umstellung auf eine vollständig elektronische Durchführung für zentrale Beschaffungsstellen seit dem 18. April 2017, für alle anderen öffentlichen Auftraggeber ab dem 18. Oktober 2018 (Höß et al., 2018, S. 40 f.).

Für Vergabeverfahren über Liefer- und Dienstleistungen im nationalen Bereich (Unterschwelle) sieht $\S 38$ Abs. 2 und $3 \mathrm{UVgO}$ bis auf wenige Ausnahmen vor, dass öffentliche Auftraggeber ab dem 01. Januar 2019 die Einreichung von Teilnahmeanträgen und Angeboten in elektronischer Form akzeptieren müssen und ab dem 01. Januar 2020 die ausschließlich elektronische Kommunikation vorschreiben. Dies gilt jedoch nicht für Aufträge unterhalb eines Auftragswerts von 25.000 Euro netto oder wenn eine Auftragsbekanntmachung nicht vorgeschrieben ist (Bundesministerium für Wirtschaft und Energie, 2019a). Für Vergabeverfahren über Bauleistungen gilt $\S 11 \mathrm{VOB} / \mathrm{A}$, der die elektronische Kommunikation zwar zulässt, aber nicht vorschreibt und auch keine Termine zur verpflichtenden Abgabe elektronischer Teilnahmeanträge und Angebote oder zur ausschließlich elektronischen Kommunikation im Vergabeverfahren vorsieht.

Wichtig zu betonen ist also, dass im Unterschwellenbereich maximal Lieferund Dienstleistungen im Rahmen der Ausschreibung (nur ein Teilprozess des gesamten öffentlichen Einkaufs) elektronisch verbindlich abgewickelt werden müssen und dies nur in jenen Ländern, in denen die UvGO durch Einführungserlasse eingeführt wurde. Das heißt, es wird nur ein sehr beschränkter Anteil aller Daten des Unterschwellenbereichs elektronisch verfügbar sein.

\subsubsection{Vergabestatistikverordnung}

Nach der VergStatVO auf Basis von $§ 114$ Abs. 2 GWB sind öffentliche Auftraggeber verpflichtet, bestimmte Daten zu öffentlichen Aufträgen zu Beschaffungs- 
vorgängen im Oberschwellenbereich ( $\$ 3$ VergStatVO und Anlagen 1-7 der VergStatVO je nach Auftraggeber) und eingeschränkt auch im Unterschwellenbereich an das BMWi zu übermitteln ( $\$ 4$ VergStatVO).

Die zu übermittelnden Daten des Oberschwellenbereichs entsprechen jenen im Formular zur Mitteilung über vergebene Aufträge. Sie sind nach der Zuschlagserteilung im EU Amtsblatt beziehungsweise in der TED zu veröffentlichen (Europäische Union, 2019b). Für den Unterschwellenbereich müssen für Vergaben oberhalb von 25.000 Euro und bis zu den in $\S 106$ GWB benannten Schwellenwerten (siehe Abschnitt 6.2.1.6) folgende Angaben gemacht werden:

- ,[Postleitzahl] und E-Mail-Adresse des [öffentlichen Auftraggebers]

- Verfahrensart (öffentlich/beschränkt/freihändig/sonstiges)

- Auftragswert ohne MwSt.

- Art und Menge der Leistung (sofern quantifizierbar)

- Weitere freiwillige Angaben" (Bundesministerium für Wirtschaft und Energie, 2019e).

Diese Angaben entsprechen im Wesentlichen den aktuellen rechtlichen Vorgaben (siehe vertiefend Abschnitt 6.3.2). Auch im Falle einer Öffnung dieser begrenzten Daten wäre es nicht möglich, das Einkaufsvolumen je Lieferant zu ermitteln, da der finale Lieferant als Datum nicht einmal statistisch übermittelt werden muss.

Das BMWi ist seit 2014 dabei, für die Erstellung dieser bundesweiten Vergabestatistik ein Tool zu entwickeln, mit dem künftig elektronisch und weitgehend automatisch relevante Vergabedaten nationaler und EU-weiter Ausschreibungen erfasst und ausgewertet werden können.

Laut einer Befragung durch das Projekt zur Erhebung der Ist-Situation im Jahr 2014 seien die vorhandenen Verfahren zur Übertragung von Daten an die EU arbeitsintensiv, fehleranfällig und verbesserungswürdig. Des Weiteren werden diese Daten vorrangig erhoben, um gesetzlichen Verpflichtungen nachzukommen - sie werden jedoch nicht als strategisches Lenkungsinstrument für den Einkauf genutzt. Eine Analyse der Daten erfolgt weder nach innen gerichtet, zum Beispiel im Rahmen der Nutzung von Synergieeffekten, noch nach außen, um etwa strategische Ziele wie die Förderung von Innovationen oder Nachhaltigkeit oder die stärkere Berücksichtigung von KMUs in den Vergabeprozessen umzusetzen (Baumann et al., 2014, S. 23 ff.).

Deutlich wird auch, dass einige Experten durchaus den Mehrwert einer einheitlichen Vergabestatistik darin sehen, dass

„... systematisch erhobene, erfasste und auswertbare Daten zur transparenteren

Gestaltung der öffentlichen Beschaffung in Deutschland und politischen 
Rechenschaftslegung, zur Beantwortung parlamentarischer und Bürgeranfragen sowie auch zur Korruptionsbekämpfung beitragen (können)“ (Baumann et al., 2014, S. 30).

Noch darüber hinaus gehen die Forderungen zur Erhebung von Daten im Unterschwellenbereich (Baumann et al., 2014, S. 36 f.). Die befragten Experten seien sich einig über den Mehraufwand, der hieraus für Vergabestellen resultiere, gleichwohl sei es im Zuge umfassender Auswertungen zur Vergabe essentiell, den Unterschwellenbereich genauso wie den Oberschwellenbereich zu erfassen. Bezüglich Tiefe und Umfang der Erfassung gingen die Meinungen allerdings auseinander: Einige Experten würden sich für die gleichen Indikatoren wie für den Oberschwellenbereich aussprechen, andere nur für einen ausgewählten Umfang je nach Ziel (zum Beispiel ausgewählte Indikatoren bei EU-Statistikmeldungen, umfangreiche Indikatoren zur strategischen Steuerung). Allgemein befürworte man Bagatellgrenzen (allerdings ohne konkrete Vorschläge für diese), unterhalb derer keinerlei Erfassungen durchgeführt werden müssen. Interessant ist, dass es bereits einige Vergabestellen gibt, die Daten im Unterschwellenbereich analog jenen des Oberschwellenbereichs erfassen, wie Abbildung 6.11 zeigt.

Zu welchen Indikatoren werden von Ihrer Vergabestelle im Unterschwellenbereich Daten erfasst? (getrennt nach Kategorien)

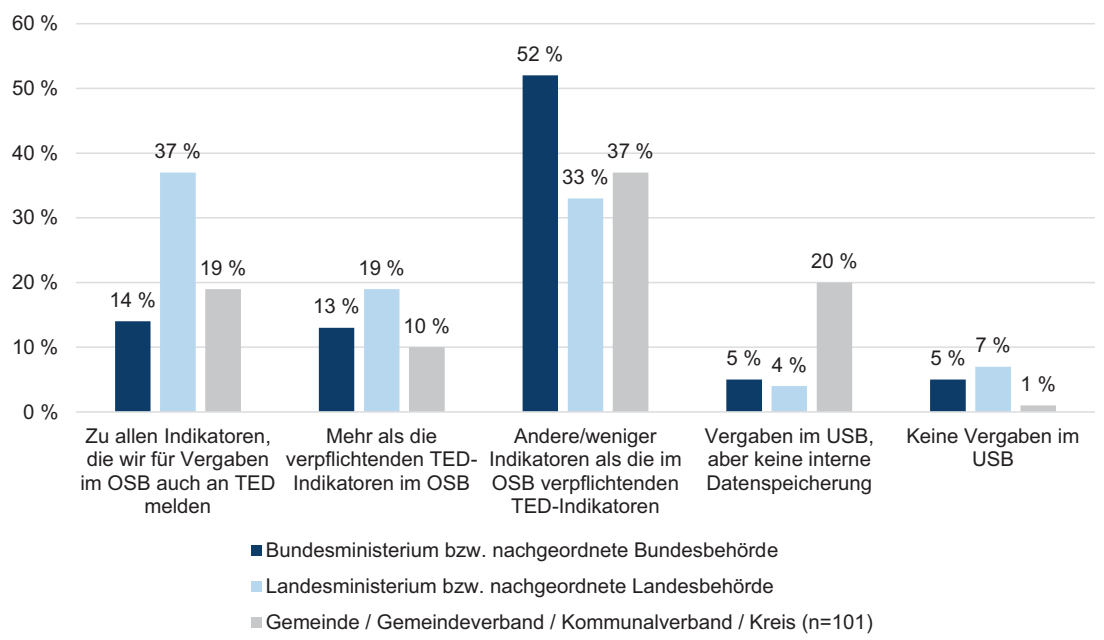

Abbildung 6.11 Erfasste Daten zu Indikatoren der TED im Unterschwellenbereich (in Anlehnung an Baumann et al., 2014, S. 64) 
Die Betrachtung getrennt nach Art der Vergabestellen ergibt ein gemischtes Bild. $14 \%$ der Bundesstellen, $37 \%$ der Landesstellen und $19 \%$ der Kommunen melden für den Unterschwellenbereich die gleichen Daten wie für den Oberschwellenbereich. $10 \%$ bis $19 \%$ der Vergabestellen geben sogar an, dass sie darüber hinaus gehen. Dies sind in Summe $27 \%$ der Stellen auf Bundesebene, $56 \%$ auf Landesebene und $29 \%$ auf kommunaler Ebene. Aus dieser Abbildung gehen nicht die Verfahrensarten hervor und daher auch nicht, ob dies nur nationale Vergaben mit EU-Charakter oder auch nationale Vergaben nach VOL/A und VOB/A betrifft. Wesentlich ist, dass die nationalen Vergaben mit EUCharakter nur einen sehr geringen Anteil einnehmen - laut Vergabestatistik des BMI aus 2017 machen diese gerade einmal knapp $5 \%$ aus ${ }^{14}$ (Bundesministerium für Wirtschaft und Energie, 2019b). Dennoch sieht man, dass durchaus ein erster Ansatz für eine Datenerhebung vorhanden wäre, der bereits teilweise - vorrangig durch Landesbehörden - genutzt wird (Baumann et al., 2014, S. 64).

Mit der automatisch erzeugten Vergabestatistik wäre es zusammengefasst möglich, eine erste Datenbasis für Bund, Länder und Kommunen für den Oberschwellenbereich (und stark eingeschränkt für den Unterschwellenbereich) zu erhalten (siehe Abschnitt 6.1).

„Erstmals kann damit zum Beispiel das jährliche Beschaffungsvolumen von Bund, Ländern und Kommunen und dessen Verteilung auf Liefer-, Dienst- und Bauleistungsaufträge verlässlich ermittelt werden“ (Bundesministerium für Wirtschaft und Energie, 2019d).

Jedoch muss auch festgestellt werden, dass die Chancen der neuen Vergabestatistik mit Blick auf weitergehende Einsatzwecke und Potentiale für den Unterschwellenbereich leider weitgehend ungenutzt geblieben sind.

Die technische und organisatorische Umsetzung der Vergabestatistik erfolgt durch das Statistische Bundesamt (Destatis). Die Inbetriebnahme der Vergabestatistik ist für das Jahr 2020 geplant. Bis zum Einsatz des neuen Vergabestatistiktools gilt $\S 8$ VergStatVO. Hiernach müssen öffentliche Auftraggeber jährlich eine Aufstellung über die im Vorjahr vergebenen öffentlichen Aufträge an das BMWi übermitteln. Dies erfolgt aufgeschlüsselt nach Liefer-, Dienstleistungs- und Bauaufträgen, nach den einzelnen Vergabearten, den

\footnotetext{
${ }^{14}$ Eigene Berechnung basierend auf den Zahlen für 2017, die am 04.März 2019 durch das Bundesministerium für Wirtschaft und Energie veröffentlicht wurden (Bundesministerium für Wirtschaft und Energie, 2019b): Im Ergebnis sind dies etwa 2 Milliarden Euro von insgesamt 40 Milliarden Euro (5\%).
} 
Auftragsgegenständen anhand der CVP-Codes sowie nach der Staatsangehörigkeit des erfolgreichen Bieters. Dies umfasst auch mindestens den Wert und die Anzahl der vergebenen Aufträge (Bundesministerium für Wirtschaft und Energie, 2019d).

\subsection{Organisatorische Umsetzung des öffentlichen Einkaufs}

\subsubsection{Organisationsformen des öffentlichen Einkaufs}

Der öffentliche Einkauf beschafft Dienstleistungen und Produkte für seine Bedarfsträger. Dabei kann er auf unterschiedliche Organisationsformen zurückgreifen, je nachdem, welcher Grad an organisatorischer (De-)Zentralisierung sinnvoll erscheint - insbesondere vor dem Hintergrund eigener Ressourcen -, wie hoch der Standardisierungsgrad der zu beschaffenden Dienstleistungen und Produkte ist, wie ausgeprägt die Prozessformalisierung ist und inwieweit Spezialkenntnisse erforderlich sind. So können sechs wesentliche Varianten skizziert werden ${ }^{15}$ (siehe auch Abbildung 6.12): Dies sind die Beschaffung über eigene Vergabestellen, welche als zentrale organisatorische Einheit intern bei der jeweiligen Behörde angesiedelt sind und ausschließlich für diese beschaffen (Hattenhauer \& Butzert, 2016), oder der Einkauf über eine punktuelle Zusammenarbeit als Zusammenschluss öffentlicher Auftraggeber, ohne dass ein Dritter, etwa eine zentrale Beschaffungsstelle, dazwischengeschaltet ist (zum Beispiel bei der Beschaffung von Spezialbedarfen für die Feuerwehr, Krankenhäuser oder Schulen). Sofern Synergieeffekte über eine größere Bündelung der Bedarfe erzielt werden sollen, ist dies mittels zentraler, institutionalisierter Beschaffungsstellen der öffentlichen Hand gemäß § 120 Abs. 4 GWB denkbar $^{16}$. Ihre Aufgabe in Form eines Eigenbetriebs, einer Eigengesellschaft oder eines rechtlich unabhängigen Zweckverbands ist die Deckung der Bedarfe der beteiligten, öffentlichen Auftraggeber. Die zentrale Beschaffungsstelle muss

\footnotetext{
${ }^{15}$ Eine Bewertung unter kartellrechtlichen oder wettbewerbsbeschränkenden Gesichtspunkten ist nicht Teil der Arbeit.

${ }^{16}$ Dies sind zum Beispiel das Kaufhaus des Bundes, das Bundesamt für Wehrtechnik und Beschaffung (BWB); das Beschaffungsamt des Bundesministeriums des Inneren (BeschA), das Beschaffungsamt der Bundeszollverwaltung (BABZV), das Gebäudemanagement Schleswig-Holstein (GMSH), das Logistikzentrum Baden-Württemberg (LZBW).
} 


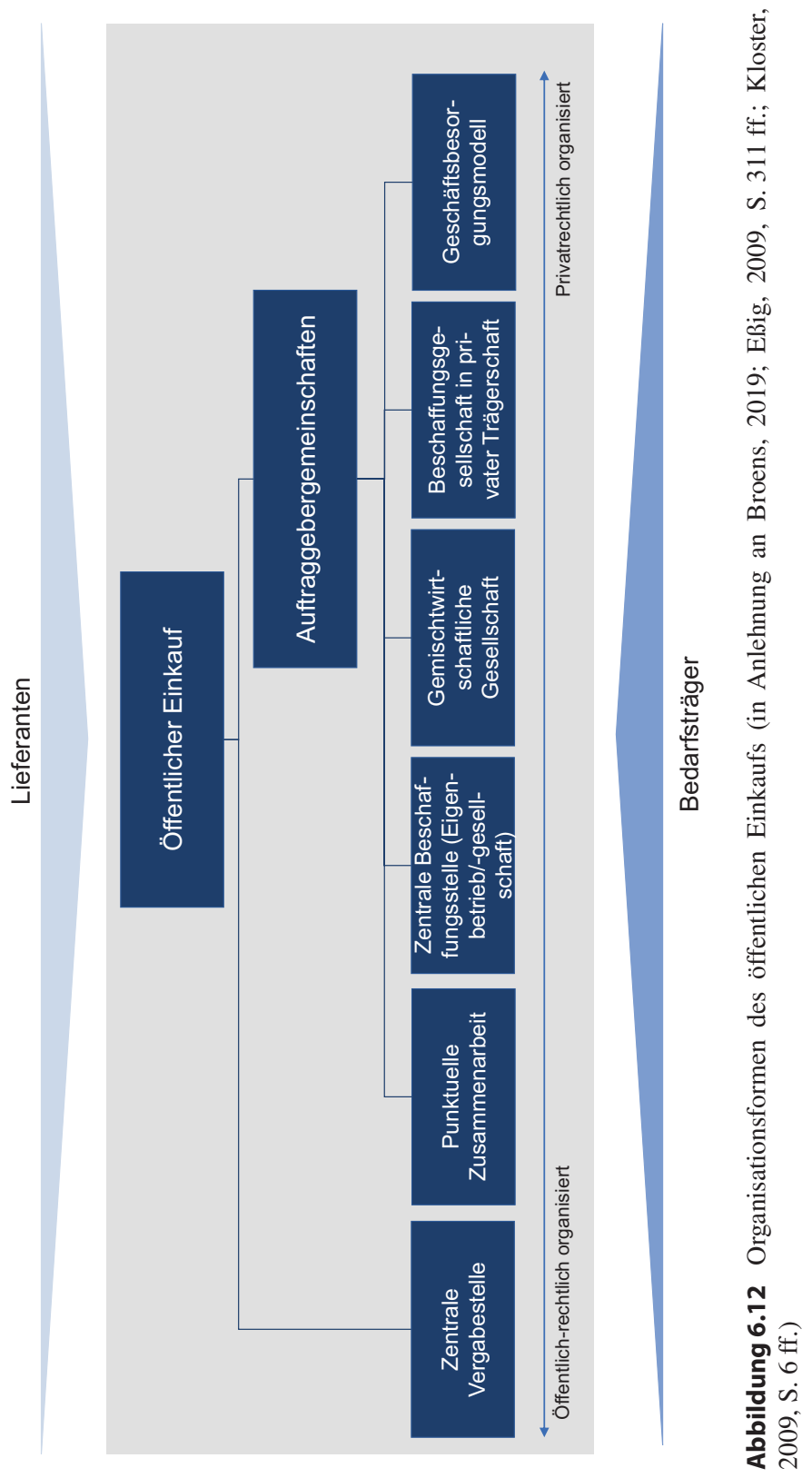


dann die Rechtmäßigkeit des Vergabeverfahrens einhalten und es muss nach außen erkennbar sein, dass sie als zentrale Beschaffungsstelle tätig wird. Sie ist entweder öffentlich-rechtlich oder privatrechtlich mit $100 \%$ Besitz durch die öffentliche Hand organisiert. Im Falle einer gemischtwirtschaftlichen Gesellschaft handelt es sich um eine zentrale Vergabestelle in privatrechtlicher Form. Die Gesellschafter sind sowohl öffentliche Auftraggeber als auch private Investoren und die Gesellschaft handelt überwiegend für die öffentliche Hand. Auch unabhängige zentrale Beschaffungsstellen können als private Einkaufsagenturen als Vertreter der Kommunen oder als Zwischenhändler tätig werden. Ein Beispiel hierfür ist die TEK-Service $A^{17}$ (TEK-Service AG, 2019). Zu guter Letzt sind unabhängige Beschaffungsstellen zu nennen, die punktuell unterstützend und beratend als privatrechtlich organisierte Geschäftsbesorger tätig werden (Broens, 2019; Christmann et al., 2004, S. 27 ff.; Eßig, 2009, S. 311 ff.; Kloster, 2009, S. 6 ff.). Die (nicht abschließenden) Varianten geben einen Eindruck der Vielfalt der beschaffenden und datenerhebenden Stellen.

\subsubsection{Der öffentliche Einkaufsprozess und Anwendung auf diese Arbeit}

Die gemeinsame Basis für alle Organisationsformen ist der öffentliche Einkaufsprozess. Vorliegende Arbeit orientiert sich an der Definition gemäß OCDS:

„For public procurement OCDS defines a unique contracting process as: All the planning, tendering information, awards, contracts and contract implementation information related to a single initiation process. An initiation process may be a tender, a direct contract award, or a call to award a concession" (Open Contracting Partnership, 2019b).

Demnach besteht der Gesamteinkaufsprozess aus fünf Schritten: Planung, Ausschreibung, Bewertung/Vergabe, Vertrag und Ausführung. Dies wird hier leicht modifiziert zu: Planung, Ausschreibung, Bewertung, Vergabe/Vertrag und Ausführung (siehe Abbildung 6.13). Die Vergabe wird dem Vertrag zugeordnet und die Bewertung als eigener Prozessschritt betrachtet, weil für die Transparenz der Prozessschritt der Bewertung inklusive Offenlegung der Selektionskriterien und Vergabeentscheidung sehr wichtig ist (Transparency International e. V., 2005).

\footnotetext{
${ }^{17}$ Die TEK-Service AG ist ein seit 2000 bundesweit tätiger Einkaufsanbieter für Verwaltungen, Organisationen und deren Dachverbände (TEK-Service AG, 2019).
} 
Gesamtprozess des öffentlichen Einkaufs

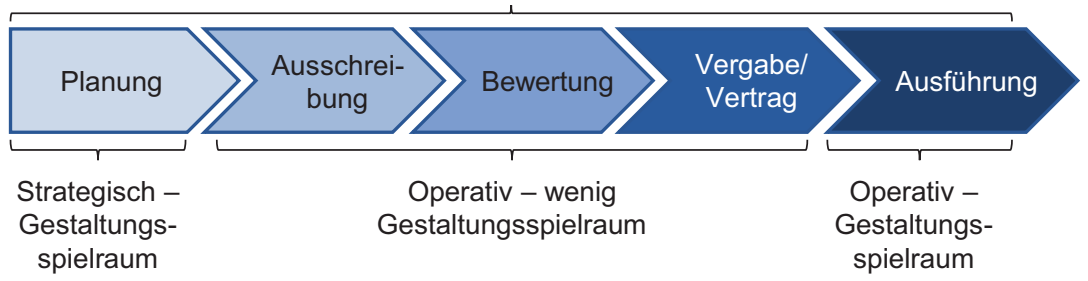

Abbildung 6.13 Gesamtprozess des öffentlichen Einkaufs

Es handelt sich um einen übergreifenden Prozess; angrenzende Subprozesse wie die Lieferantenentwicklung oder die Gestaltung von Logistikprozessen, zum Beispiel Disposition, Transport und Lagerung, werden hier nicht weiter aufgeführt, da sie für die Arbeit nicht relevant sind. Deutlich wird, dass die Vergabe nur einen Ausschnitt des gesamten Prozesses von der Planung der Bedarfe bis hin zur Leistungserbringung darstellt: Sie fokussiert auf die operative, transaktionale Abwicklung von Ausschreibungen (hier die Prozessabschnitte Ausschreibung, Bewertung und Vergabe/Vertrag) (Eßig, 2008, S. 301).

Der alleinige Fokus auf diesen Ausschnitt wäre jedoch unter dem Blickwinkel einer Öffnung des öffentlichen Einkaufs zu begrenzt, da er den Gesamtzyklus der Beschaffung vernachlässigen würde. So können sich gerade bei der Planung der Bedarfe sowie der Kontrolle der Ausführung erhebliche Einflussund Optimierungsmöglichkeiten bieten (Christmann et al., 2004, S. 24; Eßig et al., 2013, S. 26 f.; Institut für den öffentlichen Sektor e. V., 2017, S. 21). Diese Prozessabschnitte sind jene mit dem größten Risiko für Korruption (Heggstadt \& Froystad, 2011, S. 2; Transparency International e. V., 2014b, S. 21).

Des Weiteren werden den Prozessschritten insgesamt 31 Datenpunkte (siehe Tabelle 6.10) zugewiesen, die - zumindest in nicht-elektronischer Form - vorliegen (sollten), da sie zum jeweiligen Zeitpunkt erzeugt wurden. Auf diese Punkte wird in der Portalanalyse (siehe Abschnitt 7.3.9) und in der Online-Befragung (siehe Kapitel 8) Bezug genommen. 
Tabelle 6.10 Abgleich der in dieser Arbeit genutzten Daten mit dem reduzierten Datenschema aus Digiwhist (unter Bezug auf Mendes \& Fazekas, 2018, S. 18)

\begin{tabular}{|c|c|}
\hline Daten & Verwendung \\
\hline \multicolumn{2}{|l|}{ Planung } \\
\hline Bedarfsanalyse & Nur hier \\
\hline Marktanalysen & Nur hier \\
\hline Wirtschaftlichkeitsberechnung & Nur hier \\
\hline \multicolumn{2}{|l|}{ Ausschreibung } \\
\hline Beschreibung der Ausschreibung & Nur hier \\
\hline $\begin{array}{l}\text { Vergabestelle/Auftraggeber (aggregiert Abteilung, Adresse, ID, Typ aus } \\
\text { Digiwhist) }\end{array}$ & In beiden \\
\hline Ort der Leistungserbringung/Ausführungsort & Nur hier \\
\hline Verfahrensart & In beiden \\
\hline Vergabeverfahren/Vertragsart/Art des Auftrags & In beiden \\
\hline Eignungskriterien & Nur hier \\
\hline Zuschlagskriterien & In beiden \\
\hline Teilnahmefrist & In beiden \\
\hline Angebotsfrist & In beiden \\
\hline Ausschreibungs-/Vergabeunterlagen & Nur hier \\
\hline Einkaufsrichtlinien und Gesetze & Nur hier \\
\hline Einkaufsmethode (zum Beispiel Rahmenvertrag) & In beiden \\
\hline Vertragslaufzeit & In beiden \\
\hline Anzahl der ausgeschlossenen Bieter & Nur in Digiwhist \\
\hline Exakte Zeit der Angebotseinreichung & Nur in Digiwhist \\
\hline Eigentümer & Nur in Digiwhist \\
\hline Eindeutige Ausschreibungsidentität & Nur in Digiwhist \\
\hline CPV Code (\% Auftragsvolumen/Produkt) & Nur in Digiwhist \\
\hline NUTS Code(s) der Vertragsausführung & Nur in Digiwhist \\
\hline Status (zum Beispiel storniert, im Prozess) & Nur in Digiwhist \\
\hline Veröffentlichungsdatum der Ausschreibung & Nur in Digiwhist \\
\hline \multicolumn{2}{|l|}{ Bewertung } \\
\hline Basisinformationen über Mitbietende & Nur hier \\
\hline Eignung der Mitbietenden & Nur hier \\
\hline
\end{tabular}


Tabelle 6.10 (Fortsetzung)

\begin{tabular}{|c|c|}
\hline Daten & Verwendung \\
\hline Anzahl und Höhe eingegangener Angebote & In beiden \\
\hline Gewichtung der Zuschlagskriterien & Nur hier \\
\hline \multicolumn{2}{|l|}{ Vergabe } \\
\hline Finaler Vertrag inkl. Produkte/Leistungen & Nur hier \\
\hline Finaler Lieferant (aggregiert ID, Steuer-ID, Adresse aus Digiwhist) & In beiden \\
\hline Zuschlagshöhe/Vertragsvolumen & In beiden \\
\hline Finale Konditionen und Zahlungsbedingungen & Nur hier \\
\hline Veröffentlichung des Vertragszuschlags & Nur in Digiwhist \\
\hline Veröffentlichungsdatum der Vertragsvergabe & Nur in Digiwhist \\
\hline Veröffentlichungsdatum der Vertragsunterzeichnung & Nur in Digiwhist \\
\hline Veröffentlichungsdatum der Vertragsfertigstellung & Nur in Digiwhist \\
\hline Unterauftragnehmer (Name und Identität) & Nur in Digiwhist \\
\hline Anteil der Unterauftragnehmer & Nur in Digiwhist \\
\hline Mitglieder des Vergabeausschusses (Name und Identität) & Nur in Digiwhist \\
\hline Anteil der Mitglieder des Vergabeausschusses & Nur in Digiwhist \\
\hline \multicolumn{2}{|l|}{ Ausführung } \\
\hline Vertragsänderungen/Nachverhandlungen und Ergebnisse & In beiden \\
\hline $\begin{array}{l}\text { Bewertung der Leistungserbringung (fasst Vertragsenddatum, } \\
\text { Information zur Leistungsqualität und Bewertung der Vertragsleistung } \\
\text { allgemein aus Digiwhist zusammen) }\end{array}$ & In beiden \\
\hline Gesamtdokumentation des Vergabeverfahrens & Nur hier \\
\hline Überprüfung der Wirtschaftlichkeitsberechnung & Nur hier \\
\hline Mängel-/Gewährleistungsverfahren & Nur hier \\
\hline Zahlungsverkehr/Abrechnung & Nur hier \\
\hline Ergebnisse Audits, Stichproben, Revision & Nur hier \\
\hline
\end{tabular}

Die genutzten Datenpunkte gemäß Tabelle 6.10 sind weder abschließend noch ein bereits fixiertes Modell. Sie dienen vielmehr der Veranschaulichung. Die Auswahl der Daten beruht auf folgenden Überlegungen:

Im Zentrum des Interesses steht die Abbildung des Einkaufsprozesses über alle Phasen. Die praktische Abbildbarkeit in der Analyse, aber auch in der späteren Online-Befragung, muss gewährleistet werden. Aus diesem Grund wird auf ein sehr umfängliches Datenschema wie zum Beispiel jenes im OCDS mit bis zu 364 Daten verzichtet (Open Contracting Partnership, 2019i), 
sondern auf maximal 25 bis 30 Datenpunkte zurückgegriffen. Es wird nicht nach Verfahrensart beziehungsweise Vergabeverordnung unterschieden, um die Komplexität nicht unnötig zu erhöhen. Die Quellen des Datenschemas sind die juristischen Vorgaben, eigene Praxiserfahrungen sowie die Implementierungsempfehlungen des Projektes Digiwhist. Letztere enthalten ein reduziertes Datenschema mit etwa 39 Datenpunkten (Mendes \& Fazekas, 2018, S. 18).

Einige Kategorien, die bei Digiwhist dem Teilprozess der Vergabe zuzuordnen sind, wurden herausgekürzt - gleichzeitig wurde die Detaillierung begrenzt (zum Beispiel „finaler Lieferant“ ohne Präzisierung der Adresse als separates Datum dies wird impliziert.), um dafür andere Teilprozesse abzubilden, zum Beispiel die Planung und Ausführung. Im Rahmen eines gegebenenfalls nachgelagerten Projektes kann eine Betrachtung der nun ausgelassenen Daten aber durchaus sinnvoll sein.

Die erhobenen Daten im Planungsprozess geben Auskunft darüber, ob Informationen, welche zur Initiierung der Ausschreibung notwendig sind (ex-ante), veröffentlicht werden (zum Beispiel vorgeschaltete Bedarfsanalysen). Ein hoher Grad an Transparenz in diesem Bereich wäre von öffentlichem Interesse, da unabhängige Akteure überprüfen könnten, anhand welcher Kriterien die ausschreibende Stelle das Vergabeverfahren eröffnet hat.

Die Kriterien, welche im Prozessabschnitt der Ausschreibung geprüft werden, beziehen sich zunächst auf generelle Informationen der Vergabeverfahren. Es geht beispielsweise um die Bezeichnung/Beschreibung der Veröffentlichungen oder um Informationen über die Vergabestelle beziehungsweise den Auftraggeber. Des Weiteren wird in dieser Phase überprüft, welche weiteren - teilweise sensiblen Informationen bezüglich laufender Ausschreibungen veröffentlicht werden. Dazu zählen beispielsweise Eignungskriterien (wirtschaftliche/finanzielle sowie technische Leistungsfähigkeit des Bewerbers, Mindestjahresumsatz, Bilanzen, ISO-Zertifizierungen) und Fristen (Teilnahme- und Angebotsfristen). Die Daten, welche in der Ausschreibungsphase generiert werden, stellen kritische und notwendige Informationen zur Gewährleistung einer transparenten Vergabe und eines fairen Wettbewerbs dar. Vor allem für potentielle Bieter ist ihre Veröffentlichung von großem Interesse.

In der Prozessphase der Bewertung erfolgt die Prüfung der Eignung der Mitbietenden (basierend auf den zuvor kenntlich gemachten Eignungskriterien), aber auch die Anzahl und Höhe der eingegangenen Angebote und die Gewichtung der Zuschlagskriterien, die zuvor kommuniziert wurden. So ergibt sich eine nachvollziehbare und überprüfbare Bewertungsgrundlage für die Auswahl des finalen Lieferanten.

Die erhobenen Daten in der Vergabephase beziehen sich vor allem auf die ex-post Nachvollziehbarkeit. Eine hohe Transparenz in diesem Bereich ermöglicht Mitbietenden, die den Zuschlag nicht erhalten haben, zu überprüfen, wer 
den Zuschlag erhalten hat und welche Faktoren dabei für die Vergabestelle entscheidungskritisch waren. Auch für Aufsichtsorgane und Bürger wäre eine detaillierte Aufbereitung von vergebenen Aufträgen notwendig hinsichtlich der Nachvollziehbarkeit der endgültigen Entscheidungsgrundlage(n) für abgeschlossene Vergabeverfahren. Nur so kann man beispielsweise überprüfen, ob bestimmte Unternehmen in einem bestimmten Zeitraum gegenüber Mitbietenden bevorzugt werden. Alles in allem sind die Kriterien der Vergabephase mit großer Sorgfalt zu betrachten, da eine hohe Transparenz tiefe Einblicke in die detaillierte vertragsrechtliche Ausgestaltung erlaubt und der Entscheidungsprozess der Vergabestelle offengelegt wird.

Anhand der Daten, welche in dieser Phase erhoben werden, lässt sich überprüfen, ob die Annahmen, die in der Planungsphase getroffen wurden und die Ausschreibung maßgeblich beeinflussten, realistisch getroffen wurden: Sind die analysierten Bedarfe erfüllt? Ist der erstellte Business Case eingetreten? Außerdem kann in dieser Phase auch nachvollzogen werden, ob etwaige Nachverhandlungen stattgefunden haben. Eine Transparenz in diesem Bereich ermöglicht folglich Rückschlüsse auf die Leistung der Vergabestellen.

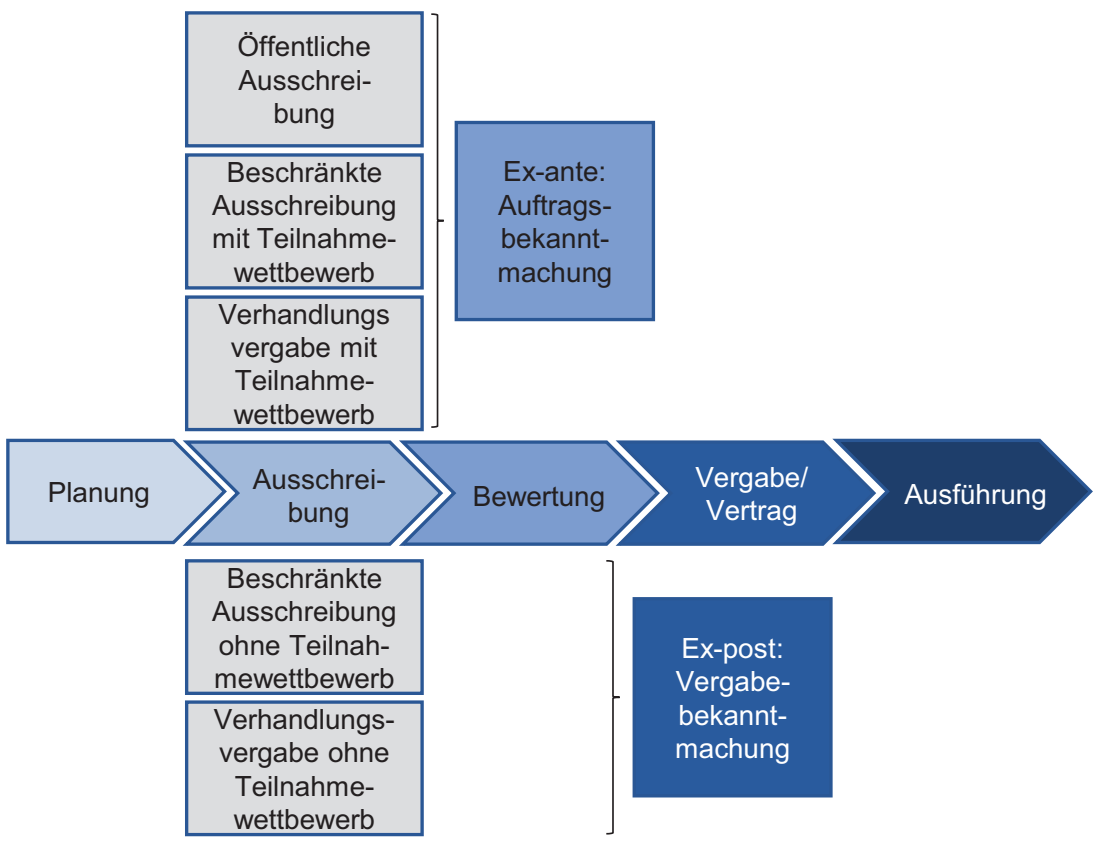

Abbildung 6.14 Zuordnung Bekanntmachungen zum Gesamtprozess des öffentlichen Einkaufs 
Die jeweiligen Daten der einzelnen Prozessschritte werden bereits veröffentlicht oder könnten in bestehenden Formaten wie der Auftrags- oder Vergabebekanntmachung veröffentlicht werden (siehe auch Abbildung 6.14). Inwieweit dies der Fall ist und welche Empfehlungen sich hieraus ergeben, wird in Abschnitt 7.3.9.4 im Rahmen der Portalanalyse näher betrachtet.

In der abschließenden Betrachtungsweise muss der Abgleich der Daten mit den juristischen Vorgaben erfolgen, um zu sehen, welche der Daten bereits heute veröffentlicht werden müssen und welche nicht. Tabelle 6.11 führt auf, welche (möglichen) Daten der Veröffentlichungspflicht unterliegen. Sofern in Klammern nicht anders dargestellt, gelten die Aussagen $(\mathrm{J}=\mathrm{Ja} ; \mathrm{N}=$ Nein) für beide Bekanntmachungstypen (Auftragsbekanntmachung, Vergabebekanntmachung).

Tabelle 6.11 Pflicht zur Veröffentlichung der Daten gemäß aktuellen Rechtsnormen

\begin{tabular}{l|l|l|l}
\hline Mögliche Daten & \multicolumn{3}{l}{ Bekanntmachung juristisch erforderlich } \\
\hline Planung & VOB/A & VOL/A & UvGO \\
\hline Bedarfsanalyse & N & N & N \\
\hline Marktanalyse & N & N & N \\
\hline Wirtschaftlichkeitsberechnung & N & N & N \\
\hline Ausschreibung & VOB/A & VOL/A & UvGO \\
\hline Beschreibung der Ausschreibung & J & J & J (N: VBM) \\
\hline Vergabestelle/Auftraggeber & J & J & J \\
\hline Ort der Leistungserbringung & J & J & J (N: VBM) \\
\hline Verfahrensart & J & J & J \\
\hline Vergabeverfahren/Vertragsart & J & J & J \\
\hline Eignungskriterien & N & N & N \\
\hline Zuschlagskriterien & N & J (N: VBM $)$ & J (N: VBM) \\
\hline Teilnahmefrist & J (N: VBM) & J (N: VBM) & J (N: VBM) \\
\hline Angebotsfrist & J (N: VBM) & J (N: VBM) & J (N: VBM) \\
\hline Ausschreibungsunterlagen & J (N: VBM) & J (N: VBM) & J (N: VBM) \\
\hline & & &
\end{tabular}


Tabelle 6.11 (Fortsetzung)

\begin{tabular}{|c|c|c|c|}
\hline \multirow{2}{*}{$\begin{array}{l}\text { Mögliche Daten } \\
\text { Einkaufsrichtlinien und Gesetze }\end{array}$} & \multicolumn{3}{|c|}{ Bekanntmachung juristisch erforderlich } \\
\hline & $\mathrm{N}$ & $\mathrm{N}$ & $\mathrm{N}$ \\
\hline Einkaufsmethode & $\mathrm{N}$ & $\mathrm{N}$ & $\mathrm{N}$ \\
\hline Vertragslaufzeit & $\mathrm{N}$ & $\mathrm{J}(\mathrm{N}: \mathrm{VBM})$ & $\mathrm{J}$ \\
\hline Basisinformationen Mitbietende & $\mathrm{N}$ & $\mathrm{N}$ & $\mathrm{N}$ \\
\hline Bewertung & VOB/A & VOL/A & UvGO \\
\hline Eignung der Mitbietenden & $\mathrm{N}$ & $\mathrm{N}$ & $\mathrm{N}$ \\
\hline Anzahl/Höhe eingegangener Angebote & $\mathrm{N}$ & $\mathrm{N}$ & $\mathrm{N}$ \\
\hline Gewichtung der Zuschlagskriterien & $\mathrm{N}$ & $\mathrm{N}$ & $\mathrm{N}$ \\
\hline Vergabe/Vertrag & VOB/A & VOL/A & UvGO \\
\hline Finaler Vertrag & $\mathrm{N}$ & $\mathrm{N}$ & $\mathrm{N}$ \\
\hline Finaler Lieferant & $\mathrm{N}(\mathrm{J}: \mathrm{VBM})$ & $\mathrm{N}(\mathrm{J}: \mathrm{VBM})$ & $\mathrm{N}(\mathrm{J}: \mathrm{VBM})$ \\
\hline Zuschlagshöhe/Vertragsvolumen & $\mathrm{N}$ & $\mathrm{N}$ & $\mathrm{N}$ \\
\hline $\begin{array}{l}\text { Finale Konditionen/ } \\
\text { Zahlungsbedingungen }\end{array}$ & $\mathrm{N}$ & $\mathrm{N}$ & $\mathrm{N}$ \\
\hline Ausführung & VOB/A & VOL/A & UvGO \\
\hline Vertragsänderungen und Ergebnisse & $\mathrm{N}$ & $\mathrm{N}$ & $\mathrm{N}$ \\
\hline Bewertung der Leistungserbringung & $\mathrm{N}$ & $\mathrm{N}$ & $\mathrm{N}$ \\
\hline Gesamtdokumentation & $\mathrm{N}$ & $\mathrm{N}$ & $\mathrm{N}$ \\
\hline Überprüfung Business Case & $\mathrm{N}$ & $\mathrm{N}$ & $\mathrm{N}$ \\
\hline Mängel-/Gewährleistungsverfahren & $\mathrm{N}$ & $\mathrm{N}$ & $\mathrm{N}$ \\
\hline Zahlungsverkehr/Abrechnung & $\mathrm{N}$ & $\mathrm{N}$ & $\mathrm{N}$ \\
\hline Ergebnisse von Audits, Stichproben & $\mathrm{N}$ & $\mathrm{N}$ & $\mathrm{N}$ \\
\hline
\end{tabular}

Legende: J: Ja, N: Nein, ABM: Auftragsbekanntmachung, VBM: Vergabebekanntmachung 
Tabelle 6.11 verdeutlicht, dass die Veröffentlichung von Daten in den Prozessschritten Planung, Bewertung und Ausführung in keiner der Vergabeordnungen und Bekanntmachungsarten vorgesehen ist. Erwartungsgemäß sind die Vorgaben im Bereich der Ausschreibung am größten, im Prozessschritt Vergabe/ Vertrag ist lediglich der finale Lieferant im Sinne der Vergabebekanntmachung vorgesehen. Auf dieses Modell werden folgend die Analysen und Ergebnisse in Abschnitt 7.3.9.4 angewandt.

\subsection{Technische Durchdringung}

\subsubsection{Die Verbreitung von public E-Procurement in Europa}

Die Ministererklärung von Manchester vom 24. November 2005 sah vor, dass bis 2010 ,alle öffentlichen Institutionen in Europa die Fähigkeit haben, 100 \% ihres Einkaufs elektronisch abzuwickeln“, und „,mindestens $50 \%$ der öffentlichen Beschaffungen mit einem Auftragsvolumen oberhalb des EU-Schwellenwerts auf elektronischem Wege durchgeführt werden“ (UK Presidency of the EU 2005, 2005, S. 4 f.). Da einige Jahre später die eVergabe trotz ambitionierter politischer Ziele immer noch bei nur $5 \%$ aller in der EU durchgeführten Vergabeverfahren lag, wurden 2010 mit dem Grünbuch (Europäische Kommission, 2010c) sowie 2012 mit der "Strategie für E-Procurement" (European Commission, 2012b) diverse Vorschläge zum Ausbau der E-Beschaffung und zur Erreichung der Ziele der Strategie EU 2020 erarbeitet. In den vergangenen Jahren wurde viel Forschung rund um die Vorteile und Nachteile betrieben ${ }^{18}$, daher sei in Tabelle 6.12 im Rahmen einer SWOT-Analyse nur auf wesentliche Aspekte verwiesen, die mit E-Procurement verbunden werden (Cattaneo \& Lifonti, 2012; European Commission, 2019b; Panda \& Sahu, 2012).

Seitdem wurden zahlreiche Maßnahmen ergriffen, um die Vorteile zu verwirklichen und die Hindernisse zu reduzieren. Einer der wesentlichen Treiber war die Vergaberechtsreform in 2016 (siehe Abschnitt 6.2.1.10), aber auch begleitende Projekte auf europäischer Ebene wie zum Beispiel die „Einheitliche Europäische

\footnotetext{
${ }^{18}$ Einen sehr guten Überblick über die Forschungsliteratur bietet Panda \& Sahu, 2012.
} 
Tabelle 6.12 SWOT-Analyse Public E-Procurement

\begin{tabular}{l|l}
\hline Stärken & Schwächen \\
\hline - Vereinfachte und verkürzte Prozesse & - Fehlender Rechtsrahmen \\
- Höhere Effizienz und Produktivität & - Fehlende Interoperabilität \\
- Kosteneinsparungen & - Starke Fragmentierung \\
- Erhöhte Transparenz & - Mangelnder Zugang zu E-Procurement- \\
- Verbesserter Wettbewerb & Systemen \\
- Korruptionsprävention & \\
- Integrierte Datenverarbeitung setzt auf \\
einer bereits vorhandenen Ist-Datenbasis \\
$\begin{array}{l}\text { und dem Open Contracting Data Standard } \\
\text { auf }\end{array}$
\end{tabular}

Eigenerklärung (ESPD; European Single Procurement Document)“, ,eCertis“ und „Pan-European Public Procurement OnLine (PEPPOL)“19 (European Commission, 2019e).

Flankierend wurde außerdem die Direktive 2014/55/EU ins Leben gerufen, welche die elektronische Rechnungsstellung vorsieht. Hiervon erhofft man sich positive Nebeneffekte auf die Automatisierung weiterer Phasen, zum Beispiel der elektronischen Bestellung, Zahlung und Archivierung (Europäische Kommission, 2013, S. 4). Immerhin konstatierte die World Bank Group im Benchmarking Report 2017, dass 154 der 180 untersuchten Länder ein E-Procurement-Portal besaßen (The World Bank Group, 2017a). Somit scheint es hier positive Veränderungen $\mathrm{zu}$ geben.

\footnotetext{
${ }^{19}$ ESPD: Die Einheitliche Europäische Eigenerklärung (ESPD) ist ein Formular für die Eigenerklärung, die in öffentlichen Beschaffungsverfahren verwendet wird. Während früher sämtliche Dokumente bereits bei der Bewerbung vorliegen mussten, ist dies nun nur noch für den Gewinner erforderlich; eCertis: Dies ist ein Web-Dienst, der übersichtlich die Anforderungen an Zertifikate und Nachweise pro Land darlegt; PEPPOL: PEPPOL ist ein internationales Projekt mit dem Ziel der Standardisierung grenzüberschreitender, elektronisch unterstützter öffentlicher Vergabeverfahren mithilfe verschiedener Applikationen innerhalb der EU (Europäische Kommission, 2019c).
} 
Es wurden demnach auf europäischer Ebene wichtige Maßnahmen ergriffen, um die Anwendung elektronischer Lösungen im öffentlichen Einkauf zu forcieren. Mit Blick auf Technologienutzung/-kompetenz, die elektronische Verwaltung und Vorhaltung von Daten sowie die Akzeptanz bei den Anwendern stellen sie eine wichtige technische Ausgangsbasis für die Öffnung des öffentlichen Einkaufs dar. Allerdings begründen die technischen Möglichkeiten, sofern vorhanden, allein noch keine Öffnung des Einkaufs: Sie sind eine notwendige, aber keine hinreichende Bedingung. Zusätzlich müssten diese Maßnahmen nicht nur auf den Oberschwellenbereich, sondern erweiternd auch auf den Unterschwellenbereich angewendet werden.

\subsubsection{Die Verbreitung von public E-Procurement in Deutschland}

Die Nutzung von E-Procurement bei öffentlichen Vergaben in Deutschland vor der Vergaberechtsreform in $2016 \mathrm{lag}$ anteilig zwischen $5 \%$ und $15 \%$ (Europäische Kommission, 2013; Langenbach, 2016). Obwohl die Vorteile von den Beteiligten gesehen wurden, war kaum Fortschritt in der Umsetzung zu verzeichnen. So gaben in einer im Jahr 2007 durchgeführten Studie die 155 befragten Körperschaften mit $92 \%$ an, die Vorteile zu erkennen, gleichwohl lag die Anwendung der elektronischen Beschaffung nur bei $30 \%$. Unter den eingesetzten elektronischen Lösungen befanden sich zu $89 \%$ die Verwendung von E-Mail, zu $65 \%$ die Nutzung elektronischer Einkaufsportale im Internet und zu $55 \%$ Preissuchmaschinen im Internet (Thome et al., 2007). Dies zeigt, dass zu diesem Zeitpunkt eher noch von partiellen statt durchgängigen Lösungen gesprochen wurde. Eine Fallstudie aus 2011 bestätigt ebenfalls, dass auf kommunaler Ebene die Vorteile des E-Procurement überwiegend wahrgenommen würden, aber davon auszugehen sei, dass E-Procurement nicht in der Breite genutzt werde, solange die bestehenden Lösungen als heterogen, unreif und teuer angesehen würden (Huntgeburth et al., 2012, S. 9).

Etwa im gleichen Zeitraum wurde das Projekt „xVergabe“ initiiert. Die „XVergabe“ ist ein im Jahr 2007 gegründetes Projekt des Landes Nordrhein-Westfalen, des Bundesverbands Materialwirtschaft, Einkauf und Logistik (BME) und des Beschaffungsamts des Bundesministeriums des Innern, für Bau und Heimat (BMI), welches sich bis zum heutigen Tag fortsetzt. Treiber hierfür war die vielfach bemängelte und fehlende Interoperabilität von mehr als 330 Vergabeportalen in der EU (Bagriantseva, 2014). Aus der Heterogenität der eingesetzten Lösungen resultierte, dass es keine Standardisierung von Soft- 
wareprodukten geben konnte und dass Bieter gezwungen waren, sich in unterschiedlichste technische Lösungen einzuarbeiten, um an allen Ausschreibungen teilnehmen zu können. Dies kann gerade bei KMUs zu einem unzumutbaren Zusatzaufwand führen und vom Bieterverfahren abhalten, was folglich den Wettbewerb reduziert. Das Projekt „xVergabe“ hatte aus diesem Grund zum Ziel, einen einheitlichen Bieterzugang zu den unterschiedlichen Vergabeportalen der öffentlichen Hand zu schaffen. Es wurde ein plattformübergreifender Datenund Austauschstandard definiert (Karich, 2017). Diese Schnittstelle wurde 2015 im Rahmen der Maßnahme „E-Beschaffung“ als Bestandteil des Regierungsprogramms „Digitale Verwaltung 2020“ als nationaler Standard bestätigt (Beschaffungsamt des Bundesministeriums des Innern, 2015; Geschäftsstelle IT-Planungsrat im Bundesministerium des Innern, 2019a). Mit einer einzigen Anwendung wäre damit die Teilnahme an Vergabeverfahren von Bund, Ländern und Kommunen möglich. Im Oktober 2019 wurde schließlich durch den ITPlanungsrat bekannt gegeben, dass die bisherige Arbeitsstruktur ausgesetzt wird und eine neue Beschlussvorlage zu erarbeiten ist (Deutsches Vergabenetzwerk $\mathrm{GmbH}, 2019)$. Das bedeutet, dass der Standard immer noch nicht flächendeckend zur Anwendung bereitsteht.

Nachdem die elektronische Vergabe schrittweise ab April 2016 eingeführt wurde, ging man zum Jahresende 2016 davon aus, dass etwa zwei Drittel aller Vergabestellen elektronische Kommunikationsmittel verwenden. Allerdings wurden überwiegend Bekanntmachungen und Vergabeunterlagen elektronisch bereitgestellt. Nur in einem Drittel der Verfahren mussten Angebote elektronisch eingereicht werden (Langenbach, 2016). Der gesamtheitliche Austausch der Unterlagen ist ab Oktober 2018 für den Oberschwellenbereich verpflichtend, ab spätestens Januar 2020 für den Unterschwellenbereich (siehe Abschnitt 6.2.1.10). Insgesamt lässt sich feststellen, dass das Prinzip der Freiwilligkeit, welches bis zur Vergaberechtsreform im Jahr 2016 galt, wenig Fortschritt nach sich gezogen hat. Erst die „,von oben“ verordnete Reform hat für die notwendigen (ersten) Veränderungen im Oberschwellenbereich gesorgt. Aktuell fehlen allerdings Zahlen zum Status quo der gesamthaften Anwendung elektronischer Mittel sowohl im Ober- als auch im Unterschwellenbereich. 


\subsection{Transparenz, Korruption, Partizipation und Kollaboration}

Die Idee des Open Government mit den Grundpfeilern Transparenz, Partizipation und Kollaboration (siehe Abbildung 6.15) geht mit einem Perspektivenwechsel einher hinsichtlich der Art und Weise, wie Staat, Verwaltung und Bürger miteinander kommunizieren.

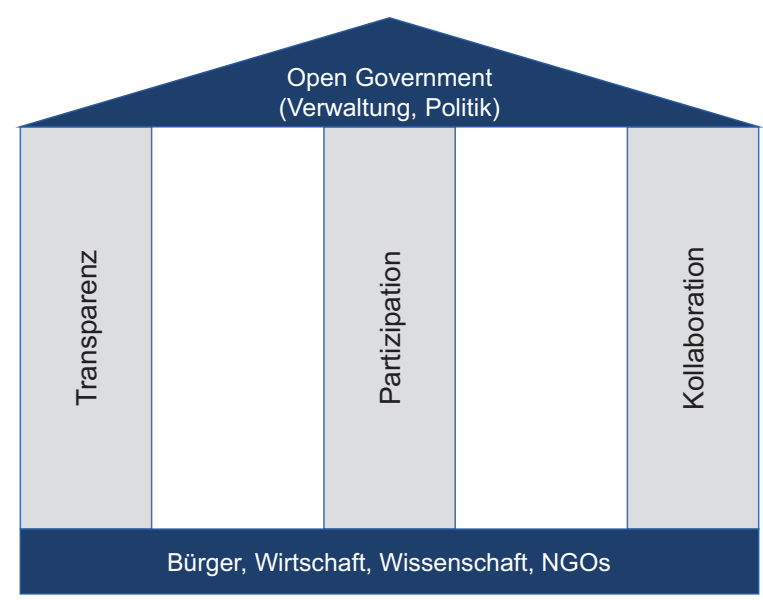

Abbildung 6.15 Drei Säulen des Open Government (in Anlehnung an Leitner, 2018, S. 13)

Transparenz, Partizipation und Kollaboration seien, so Martini

,als Dreiklang innovativer Governance komponiert, dessen Partitur die moderne Verwaltung der Zukunft orchestrieren soll. Der transparente Staat legt seine Entscheidungsgrundlagen offen, er bezieht die Bürger in die Entscheidungen ein und generiert durch das Zusammenwirken einen Mehrwert für das Gemeinwohl“ (Martini, 2014, S. 13).

In dieser Vision sorgt Transparenz für die Offenlegung politischer Entscheidungsfindung und Information der Bürger, die hierüber einbezogen werden. Die Partizipation einzelner Bürger verbreitert das Wissen in der Bevölkerung und erhöht die Qualität der Entscheidungen. So kann eine neue Kultur des Zusammenarbeitens, der Kollaboration, zwischen Staat und Gesellschaft entstehen. 
Im Folgenden wird hierauf mit Bezug auf den öffentlichen Einkauf eingegangen. Korruption ist dabei eines der am meisten diskutierten Problemfelder, was sich nicht nur aus den zuvor beschriebenen Prinzipien des Vergaberechts ergibt, sondern auch aus der Wissenschaft (Beck, 2012, S. 41 ff.) und Befragungen der Öffentlichkeit. So waren in einer Umfrage aus dem Jahr 2012 für $95 \%$ der Befragten strafrechtliche Konsequenzen für Amtsträger, die ihre Position missbrauchen, wichtig, für $94 \%$ Regeln und Maßnahmen zur Korruptionsbekämpfung in Politik und Verwaltung und für $91 \%$ Informationen über das Ausmaß von Korruption, Amtsmissbrauch und persönlicher Vorteilsnahme (Open Government Partnership Deutschland e. V., 2012, S. 9).

\subsubsection{Der Zusammenhang zwischen Transparenz und Korruption}

Da Transparenz und Korruption gern eine Wechselwirkung zugeschrieben wird (mehr Transparenz wirkt korruptionsvermeidend, weniger Transparenz fördert korruptes Handeln), soll in diesem Kapitel hierauf mit Bezug auf den öffentlichen Einkauf näher eingegangen werden.

\subsubsection{Definition von Transparenz und Korruption}

Der Begriff der Transparenz setzt sich zusammen aus trans (lat. darüber, hinüber, jenseits, durch) und parere (lat. scheinen). Der Duden führt zahlreiche Synonyme für Transparenz an: Durchscheinen, Durchsichtigkeit, Lichtdurchlässigkeit, Aufrichtigkeit, Durchschaubarkeit, Geradheit, Geradlinigkeit, Nachvollziehbarkeit und Offenheit (Bibliographisches Institut GmbH (Duden), 2019b). Transparenz aus Sicht der Verwaltungswissenschaften bedeutet, „Vorgänge und Entscheidungen in Politik, Verwaltung und Justiz von außen nachvollziehbar zu machen" (von Lucke, 2012a, S. 60). Eine höhere Transparenz kann die Steuerbarkeit vereinfachen, Korruption reduzieren und zu einem verbesserten Preis-/Leistungsverhältnis sowie einer höheren Legitimität des politischen Systems führen (Kaufmann, 2005, S. 92). ${ }^{20}$

\footnotetext{
${ }^{20}$ Dies kann sich zum Beispiel in öffentlichen Debatten des Bundestags, in der Informationsfreiheit oder öffentlichen Gerichtsprozessen ausdrücken. Als Gegenspieler der Transparenz werden oftmals Amts- und Geschäftsgeheimnisse angeführt, deren Schutz ein besonderes Interesse erfährt (von Lucke, 2010, S. 397 f.).
} 
Mit Blick auf die Debatte rund um ein offenes Regierungs- und Verwaltungshandeln wird Transparenz vorwiegend mit der Veröffentlichung von Datenbeständen des öffentlichen Sektors in Verbindung gebracht mit dem Ziel, eine höhere Verantwortungsbereitschaft zu erzeugen. Darüber hinaus soll Transparenz mithilfe geeigneter Mechanismen Partizipation und Kollaboration ermöglichen oder umgekehrt positiv auf die Verantwortungsübernahme einwirken (Janda, 2011, S. 21 f.; Transparency International e. V., 2014b, S. 22).

Negative Effekte von Transparenz sind die Kosten der Aufbereitung und Bereitstellung von Informationen im Verhältnis zu ihrem Nutzen (Coppier \& Piga, 2006), die Abwanderung von Korruption in neue oder intransparente Bereiche (Bundesverband Materialwirtschaft \& Beschaffungsamt des Bundesministeriums des Innern, 2004), die mögliche Reduktion von Wettbewerb (Graells, 2013) und die Offenlegung von Mechanismen, die zur ungewollten Nachahmung führen können (Bac, 2001, S. 93). Es gibt offenbar ein Spannungsfeld zwischen Offenheit und Zurückhaltung von Informationen, das einen reflektierten Umgang erfordert.

Korruption (von lat. corruptio) bezeichnet Bestechlichkeit, Bestechung, Vorteilsannahme und Vorteilsgewährung (Bibliographisches Institut GmbH (Duden), 2019a). TI definiert Korruption als „Missbrauch anvertrauter Macht zum privaten Nutzen oder Vorteil“" (Transparency International Deutschland e. V., 2019a). Es bedeutet, dass das Wohl des Einzelnen über das Wohl der Allgemeinheit gestellt wird. Unter Korruption im Vergaberecht werden die Situationen verstanden, in denen ein Teilnehmer im Vergabeverfahren einem Korruptionspartner einen Vorteil gewährt, um einen öffentlichen Auftrag jenseits der vergaberechtlichen Prinzipien zu erhalten. Der Empfänger dieser Vorteile kann dabei der öffentliche Auftraggeber (öffentliche Korruption), aber auch ein Konkurrent im Verfahren sein (private Korruption) (Gentsch, 2012, S. 66).

Ziel von Vertretern der Zivilgesellschaft wie NGOs ist die Bekämpfung der Korruption aufgrund ihrer negativen immateriellen und materiellen Folgen (siehe Abbildung 6.16) auf zum Beispiel Wachstum, Inflation, Fremdkapitalkosten, Ausgaben für Bildung und Infrastruktur und Steuereinnahmen sowie die Glaubwürdigkeit der Akteure in Politik und Verwaltung (Frank, 2004; Glennerster \& Shin, 2008; Wensink \& de Vet, 2013). Neben Politikverdrossenheit kann dies auch zu demokratie- und damit staatsfeindlichen Entwicklungen führen, die es zu verhindern gilt. Korruption verursache laut TI materielle Schäden und untergrabe „das Fundament einer Gesellschaft“ (Transparency International Deutschland e. V., 2019a).

Da der Vermeidung von Korruption somit eine hohe Bedeutung zukommt, soll sie in den folgenden Kapiteln weiter vertieft werden. 

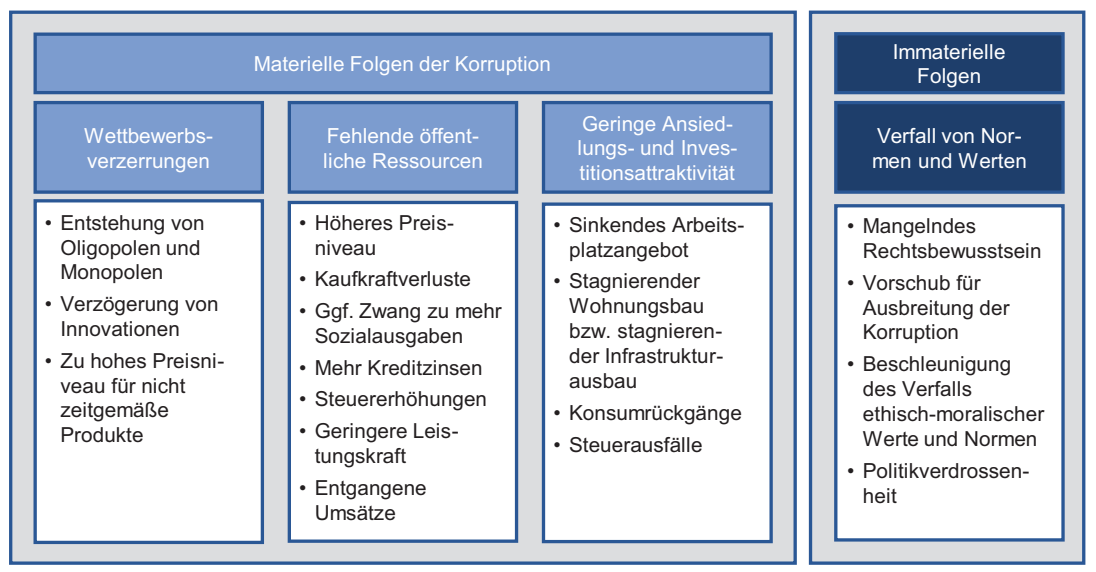

Abbildung 6.16 Materielle und immaterielle Folgen der Korruption (in Anlehnung an Arnold \& Truck, 2004, S. 13)

\subsubsection{Ursachen und Erscheinungsformen von Korruption}

Als Ursachen von Korruption können sowohl gesellschaftliche und institutionelle als auch individuelle Faktoren genannt werden, die sich wechselseitig beeinflussen oder überlappen können (Gentsch, 2012, S. 75 ff.; Heggstadt \& Froystad, 2011, S. 4 ff.; Kühn \& Sherman, 2014, S. 20). Zu den gesellschaftlichen Faktoren zählen zum Beispiel die Marktsituation und hiermit verbunden Güterknappheit oder Verteilungsprobleme sowie die Gesetzgebung mit niedrigen Rechts- und Entwicklungsstandards. Institutionelle Faktoren können auf den Aufbau und die Ablauforganisation zurückgehen: Komplexe Verwaltungseinheiten und -abläufe mit einer starken Fragmentierung, ein großer Ermessensspielraum für die Amtsträger sowie wirtschaftliche Monopole mit fehlenden Kontrollen und geringer Transparenz sind als korruptionsförderliche Faktoren zu nennen. Zu den individuellen Faktoren zählen zum Beispiel fehlendes Unrechtsbewusstsein, Leistungsdruck oder Geldnot. Je nach Situation und aufgrund der Schwierigkeit, dass Korruption zumeist im Verborgenen geschieht, muss ein Bündel an Gegenmaßnahmen ergriffen werden.

Es können im Wesentlichen folgende Erscheinungsformen unterschieden werden: Bestechung, Angebotsabsprachen, Betrug und die Nutzung informeller Netzwerke (Hanna et al., 2011, S. 8; Heggstadt \& Froystad, 2011, S. 7). Das Ausmaß der Korruption wird anhand verschiedener Indizes erhoben (Transparency International e. V. et al., 2016). Schaut man sich die Zahlen für Deutschland an, zum 
Beispiel anhand des Corruption Perceptions Index (CPI), so schneidet Deutschland im internationalen Vergleich zumeist im oberen Mittelfeld mit im Durchschnitt acht von zehn Punkten ab und ist somit eines der 20 am wenigsten von Korruption im öffentlichen Sektor betroffenen Ländern der Welt (Korczak et al., 2012, S. 18). Allerdings geben Zahlen des Bundeskriminalamts (BKA) zu denken: Demnach ist im Betrachtungszeitraum 2007 bis 2017 die Vergabe öffentlicher Aufträge im Vergleich zu allen anderen Bereichen überdurchschnittlich von Korruption betroffen und liegt mit deutlichem Abstand an erster Stelle ${ }^{21}$. Abbildung 6.17 zeigt den Anteil der Erlangung von Aufträgen an allen erlangten Vorteilen im zeitlichen Verlauf.

Anteil "Erlangung von Aufträgen" an allen Vorteilen in \% in Deutschland

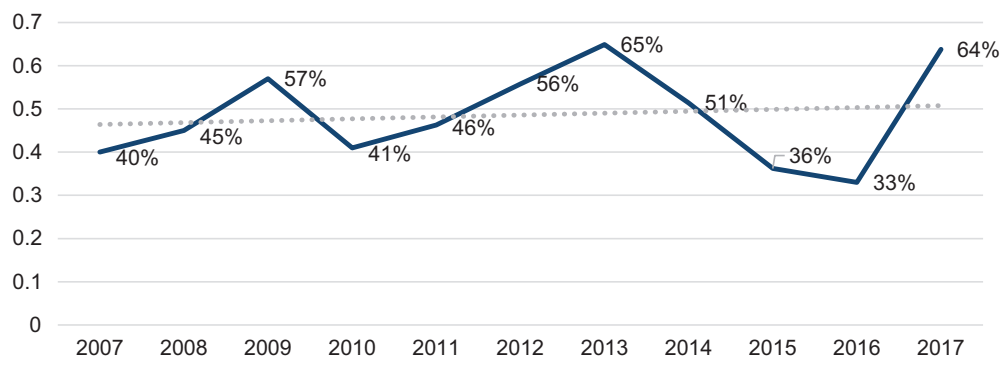

Abbildung 6.17 Anteil „Erlangung von Aufträgen“ an allen Vorteilen (in \%) (in Anlehnung an Bundeskriminalamt, 2019)

Die Empfänger der Zuwendungen sind häufig Personen aus Kommunalbehörden, Gesundheitseinrichtungen, Bildungsinstitutionen, Behörden und privaten Betrieben (Bundeskriminalamt, 2019).

Der Bribe Payers Index (BPI) ${ }^{22}$ zeigt auf, dass Korruption bei öffentlichen Bau- und Konstruktionsaufträgen an erster Stelle steht (Hardoon \& Heinrich,

\footnotetext{
${ }^{21}$ Mit deutlichem Abstand folgen (exemplarisch aus dem Jahr 2017) auf die Erlangung von Aufträgen (63,8\%): Erlangung behördlicher Genehmigungen (13,7\%), sonstige Wettbewerbsvorteile $(6 \%)$, Beeinflussung der Strafverfolgung (5\%), Erlangung interner Informationen (1,9\%) (Bundeskriminalamt, 2017, S. 18). Grundlage der Datenzusammenstellung sind Zulieferungen der Landeskriminalämter, des Bundeskriminalamts, der Bundespolizei und des Zollkriminalamts (Bundeskriminalamt, 2017, S. 2).

${ }^{22}$ Der BPI wurde alle drei Jahre beginnend mit dem Jahr 1999 bis zum Jahr 2011 von TI erhoben (Transparency International e. V., 2019a).
} 
2011, S. 15; Transparency International e. V., 2008, S. 11). Ergänzend führt eine Studie auf, dass die Korruption im öffentlichen Einkauf auf nationaler Ebene von $67 \%$ der Befragten wahrgenommen wird, auf regionaler beziehungsweise lokaler Ebene von $62 \%$ der Befragten (European Commission, 2017, S. 37 f.).

Das bedeutet, dass Antikorruptionsmaßnahmen in diesem Bereich essentiell sind und bestehende Maßnahmen möglicherweise über die letzten zehn Jahre nur punktuell zu Verbesserungen geführt haben oder ihr Effekt nicht eindeutig nachweisbar ist. Ebenfalls deutlich wird der Bedarf, Erhebungen zur wahrgenommenen Korruption mit Erhebungen zu realen Fällen zu vergleichen.

\subsubsection{Die rechtlichen Rahmenbedingungen in der Korruptionsbekämpfung}

In den letzten Jahren wurde eine Vielzahl internationaler Abkommen gegen Korruption abgeschlossen. Die meisten beinhalten Regeln für einen sicheren öffentlichen Einkauf, so die OECD Anti-Bribery Convention, die United Nations Convention against Corruption (UNCAC), die Accra-Agenda for Action, das World Trade Organisation Agreement on Government Procurement, die Direktiven des EU-Parlaments und des Councils on Procurement (Heggstadt \& Froystad, 2011, S. 5; Pyman et al., 2018, S. 11).

Auf europäischer Ebene gilt gemäß Art. 2 des Vertrags über die Europäische Union (EUV) das Prinzip der Rechtsstaatlichkeit. Dies soll mithilfe eines Raumes der Freiheit, der Sicherheit und des Rechts (RFSR) gemäß Art. 3 Abs. 2 EUV erreicht werden. Hiermit soll vor allem der europäische Binnenmarkt umgesetzt werden, welcher gemäß Art. 67 Abs. 2 Arbeitsweise der Europäischen Union (AEUV) die Vermeidung und Bekämpfung von Kriminalität vorsieht. Hierunter würde auch die Verhinderung der Korruption im öffentlichen Auftragswesen fallen. Zudem ist Art. 325 AEUV als einschlägig zu nennen, welcher die Betrugsbekämpfung zum Schutz der finanziellen Interessen der EU, auch grenzüberschreitend, vorsieht (Gentsch, 2012, S. 40 ff.).

In Deutschland gründet sich der Auftrag zur Korruptionsprävention und -bekämpfung darauf, dass der Staat die Teilnehmer und ihre Rechtsgüter im Vergabeverfahren gegen rechtswidrige Angriffe zu schützen hat. Obwohl im Grundgesetz hierzu keine Konkretisierungen enthalten sind, gehört es zu den Aufgaben eines Staates, seine Bürger vor Kriminalität zu bewahren. Hierzu finden sich im Strafgesetzbuch (StGB) die entsprechenden Straftatbestände. Weiters hat der Staat das Gemeinwesen (also die Gesellschaft als Ganzes, seine Volksund Marktwirtschaft) zu schützen. Seit 26. November 2015 gilt das Gesetz zur Bekämpfung der Korruption (KorrBekG). Der Name des Gesetzes folgt seinem Vorgänger aus dem Jahr 1997. Es bezweckt eine erweiterte Strafbarkeit der 
Korruption im geschäftlichen und privaten Sektor und zwar sowohl in Deutschland wie auch im internationalen Kontext. ${ }^{23} \mathrm{Im}$ Vergaberecht fordern die Grundsätze der Wirtschaftlichkeit und Transparenz ein Engagement in Richtung Bekämpfung der Korruption, da die öffentlichen Gelder sparsam und wirtschaftlich eingesetzt werden müssen, um die Leistungsfähigkeit des Staates gemäß Art. 20 GG sicherzustellen (Gentsch, 2012, S. 34 ff.). Bei Nichteinhaltung der entsprechenden Regeln und Bekanntwerden der Korruption kommen im Wesentlichen strafrechtliche Maßnahmen gemäß StGB (§§ 331-338) zur Anwendung (Jungclaus, 2004, S. 24; Lohner \& Behr, 2019).

\subsubsection{Korruption und Transparenz im öffentlichen Einkauf}

Korruption kann entlang des gesamten Prozesses entstehen. Anders ausgedrückt: Es ist nicht möglich, Korruption in einer der Einkaufsphasen auszuschließen und sie kann sehr unterschiedliche Formen annehmen (Gentsch, 2012, S. 67 ff.; Heggstadt \& Froystad, 2011, S. 20 ff.; Korczak et al., 2012; Kühn \& Sherman, 2014, S. 22 ff.; OECD, 2007a, S. 20; Rozo, 2010). In Tabelle 6.13 ist eine Reihe an Beispielen für Korruptionsrisiken den möglichen Gegenmaßnahmen gegenübergestellt.

Informations- und Kommunikationstechnologien können ein Transmissionsriemen zur vereinfachten Herstellung von Transparenz entlang des Einkaufsprozesses sein - viele der in Tabelle 6.13 aufgeführten Gegenmaßnahmen lassen sich hiermit direkt umsetzen (zum Beispiel Anwendung eines Archivierungssystems) oder besser nutzbar machen (zum Beispiel der Einsatz eines Online-Wettbewerbsregisters, die zentrale Bereitstellung von Informationen) (OECD, 2007b). Allerdings muss sorgfältig reflektiert werden, welche Art der Information bereitgestellt wird und wer welchen Zugriff hierauf hat (OECD, 2007a, S. 55). Letztlich ist die Anwendung von IKT auch kein Allheilmittel gegen Korruption: Sie vermag zwar die Korruption des papiergestützten Verfahrens einzugrenzen; da Korruption aber immer von den handelnden Personen ausgeht, ist für eine nachhaltige und durchgängige Wirksamkeit das

\footnotetext{
${ }^{23}$ Das Gesetz dient ,im Wesentlichen der Umsetzung internationaler Vorgaben zur Bekämpfung der Korruption und soll Strafbarkeitslücken, etwa beim Geldwäschetatbestand (§ 261 StGB), schließen. Um mehr Übersichtlichkeit zu gewährleisten, wurden die vorhandenen Korruptionsvorschriften des IntBestG und des EUBestG in das StGB überführt, ferner die Korruptionsstrafbarkeiten im geschäftlichen Verkehr ( $\$ 299$ StGB) grundlegend erweitert und die Strafbarkeit im öffentlichen Sektor ( $\S \S 331,333$ StGB) auf europäische Amtsträger ausgedehnt. Künftig wird zudem auch die Vorteilsgewährung an ausländische Amtsträger bestraft (§ 335a StGB)“ (Ahlbrecht \& Dann, 2015).
} 


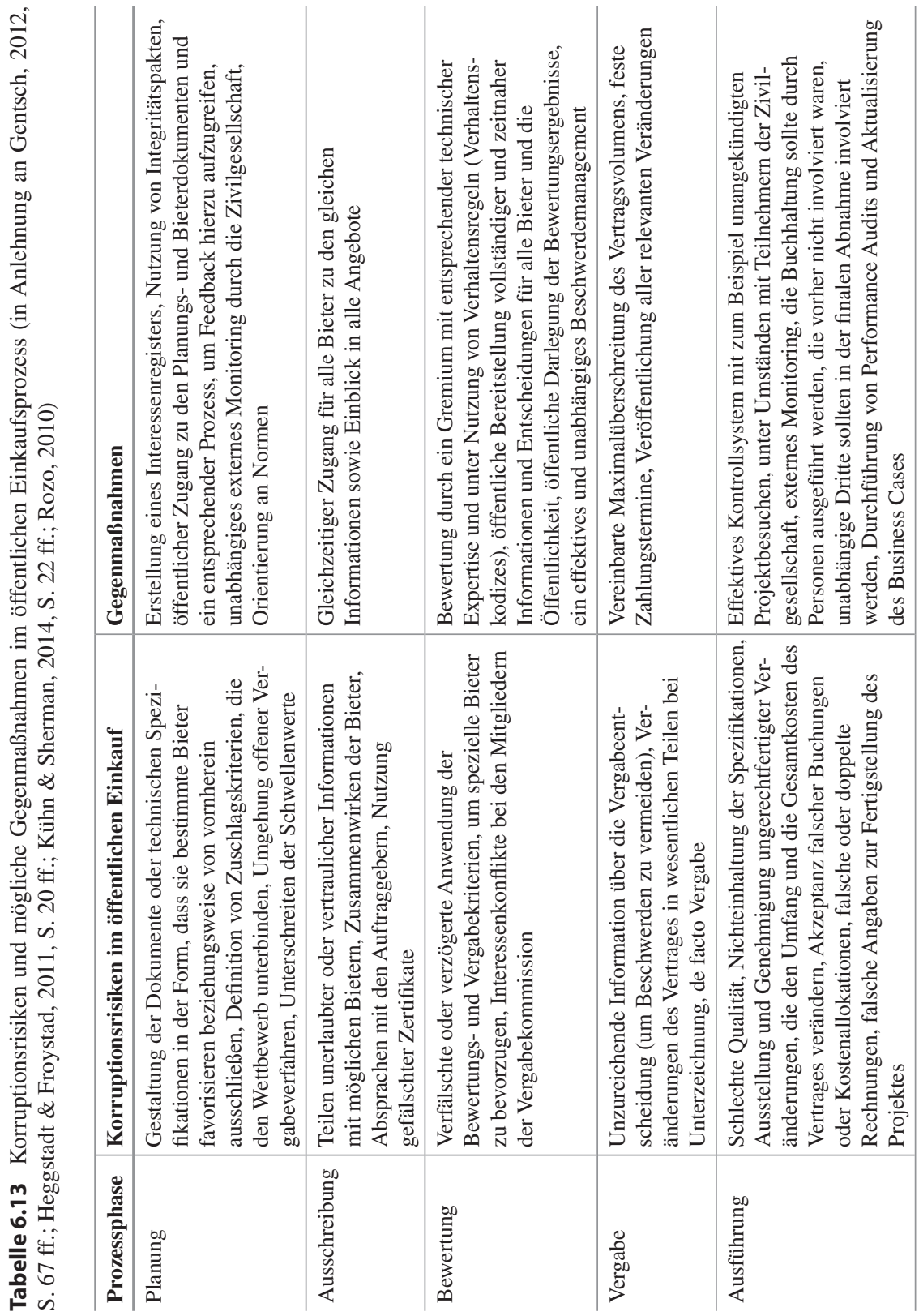


Zusammenspiel mit anderen Maßnahmen entscheidend (Fuchs \& Helfrich, 2004, S. 31; Kühn \& Sherman, 2014, S. 28). Zudem darf nicht unbeachtet bleiben, dass durch IKT neue Korruptionsmöglichkeiten entstehen können. Diese können zum Beispiel in den technischen Bestandteilen oder Schnittstellen begründet liegen oder auf die Systemadministratoren und Nutzer selbst zurückgehen (Elschner, 2004, S. 37; Hannson \& Holmgren, 2011). Korruption passt sich ihrer Umwelt an und ist damit ein sich wandelndes Phänomen, welches flexibler Lösungsansätze bedarf.

\subsubsection{Auswirkungen von Transparenz auf Korruption}

Im Folgenden wird ein kurzer Überblick über Studien gegeben, die erstens die Auswirkungen von Transparenz auf Korruption im Allgemeinen und zweitens die Auswirkungen von Transparenz auf Korruption im öffentlichen Einkauf darlegen.

Mit Bezug auf die Auswirkungen von Transparenz auf Korruption im Allgemeinen zeigt ein systematischer Review verschiedener MikroLevel-Strategien gegen Korruption (Hanna et al., 2011) einen positiven Zusammenhang zwischen Transparenz und Korruption. So konnte nachgewiesen werden, dass Korruption durch entsprechende Auditprogramme (initiiert durch eine glaubwürdige, neutrale Gruppe) reduziert werden konnte, wenn korruptes Verhalten tatsächlich sichtbare Konsequenzen nach sich zog, zum Beispiel durch die Veröffentlichung der Auditergebnisse, die Reduktion künftiger Budgets oder die Kündigung korrupter Mitarbeiter (Brollo, 2010; Olken, 2006; Savedoff, 2007). Gleiches galt, wenn zum Beispiel die Kontrollmöglichkeiten durch die Zivilgesellschaft mit einer zielgerichteten Medienkampagne oder anderen Formen der Informationsbereitstellung verbunden wurden, das heißt, wenn die Möglichkeit einer öffentlichkeitswirksamen Konsequenz bestand (Björkman \& Svensson, 2009; Reinikka \& Svensson, 2011). Gleichzeitig mahnen die Studienverantwortlichen hier aber zur Vorsicht und verweisen auf die Kultur der jeweiligen Gesellschaft (beispielsweise in Bezug auf ihren Umgang mit Fehlern) sowie die Schutzmöglichkeiten entsprechender Hinweisgeber (,whistleblower protection“). Auch die Kombination aus institutionellem Monitoring mit nicht-monetären Anreizen zeigte positive Effekte, wenngleich diese gegebenenfalls über die Zeit nachlassen könnten (Brollo, 2010). Weiters wurde Dezentralisierung als positiver Treiber gesehen, sofern dies mit einer erhöhten Beteiligung der Zivilgesellschaft über alle sozialen Klassen und Interesse an lokalen politischen Entscheidungen verbunden ist. Im anderen Fall könnte die Gefahr einer Verlagerung der Korruption von zentralen auf lokale Stellen entstehen (Björkman \& Svensson, 2009; Olken, 2006). An den Beispielen wird ersichtlich, dass ein strukturelles Element 
kombiniert mit der Offenlegung von Informationen sowie der Gegenwart oder sogar aktiven Einbindung der Zivilgesellschaft einen positiven Einfluss auf die Vermeidung beziehungsweise Reduktion von Korruption haben (hierzu noch mehr in Abschnitt 6.5.3). Auch die Resultate einer Untersuchung in Brasilien in 2004 zeigen, dass veröffentlichte Auditergebnisse einen starken Einfluss auf die Wahlergebnisse hatten und dies vor allem in Kommunen, in denen das lokale Radio diese Informationen verbreitete (Ferraz \& Finan, 2008, S. 703).

Mit Blick auf die Auswirkungen von Transparenz auf Korruption im öffentlichen Einkauf kann als ein Beispiel die Offenlegung von Preisinformationen in Argentinien herangezogen werden: Dort wurden im Jahr 1996 unter Einbeziehung von 33 Kliniken die Preise für medizinische Hilfsmittel offengelegt. In den ersten Monaten des Experiments sanken sowohl die Preise als auch die Varianz hierfür. Im weiteren Verlauf zeigte sich allerdings, dass die Preise wieder anstiegen - hier vermutete man einen Effekt der Gewöhnung an die Veröffentlichung der Informationen, kombiniert mit der Erkenntnis, dass das Verhalten keine Konsequenzen nach sich zog. Demnach hat die reine Offenlegung von Informationen ohne Folgen nur transitorische Effekte (Savedoff, 2007). Weiters zeigte Ohashi für Japan, dass die verbesserte Transparenz im Bieterqualifizierungsprozess im kommunalen Bereich zu einer Reduktion der Einkaufskosten von maximal $3 \%$ führte (Ohashi, 2008, S. 18). Für Russland analysierte man die auf Webseiten verfügbaren Informationen rund um den öffentlichen Einkauf (geplante Ausschreibungen sowie Ergebnisse der Ausschreibungen in Form von Protokollen). Hiermit konnte ebenfalls ein positiver Zusammenhang zwischen Reduktion der Korruption bei höherer Transparenz aufgezeigt werden (Balsevich et al., 2011, S. 20). Auch Bertot, Jaeger und Grimes führen diverse Beispiele auf, in denen Korruption durch mehr Transparenz in den E-Procurement-Systemen im öffentlichen Einkauf reduziert werden konnte, indem öffentliche Ausschreibungen erhöht und Festpreismechanismen reduziert wurden (Bertot et al., 2010, S. 265 f.).

Eine letzte interessante und zunächst widersprüchlich erscheinende Beobachtung ist, dass Länder, die gemäß CPI 2018 eine höhere Korruption aufweisen, sich offensichtlich stärker für eine transparentere Darstellung ihrer öffentlichen Einkaufsaktivitäten einsetzen als Länder, die eine geringere wahrgenommene Korruption besitzen. Dies betrifft zum Beispiel 13 der 18 Länder, die den OCDS bereits einführen. ${ }^{24}$ Coppier und Piga führten hierzu eine Studie

\footnotetext{
${ }^{24} \mathrm{Ab}$ einem Platz oberhalb von 57 und einem Score oberhalb von 50 Punkten sind dies Afghanistan (Platz 172), Armenien (Platz 105), Honduras (Platz 132), Indonesien (Platz 89), Kolumbien (Platz 99), Mexiko (Platz 138), Moldawien (Platz 117), Nepal (Platz 124), Nigeria (Platz 144), Paraguay (Platz 132), Sambia (Platz 105), Uganda (Platz 49) und die Ukraine (Platz 120) (Transparency International e. V., 2018b).
} 
durch und kamen zum Ergebnis, dass Korruption im öffentlichen Einkauf eine große Rolle bei der Beeinflussung der Gesamtkorruption eines Landes spielt. So wählen Länder mit einer größeren wahrgenommenen Korruption den Ansatz, die Transparenz vor allem in diesem Bereich zu erhöhen, da sie sich davon den größten Einfluss bei überschaubarem Mitteleinsatz versprechen. Die Autoren empfehlen, dass dieser Ansatz durch die Nutzung von IKT im öffentlichen Einkauf unterstützt werden kann, um so Ausschreibungen und Ergebnisse effizient $\mathrm{zu}$ publizieren und die Öffentlichkeit in das Monitoring mit einzubeziehen. „'Voice' and 'Public Governance' will thus acquire new power thanks to the quality of specifically tailored e-based solutions“ (Coppier \& Piga, 2006, S. 16).

\subsubsection{Partizipation als wesentlicher Bestandteil der Offenheit}

\subsubsection{Definition}

Die Einbeziehung von Dritten in Verwaltungsprozesse und die Mitwirkung an politisch-administrativen Planungs- und Entscheidungsprozessen sind wichtige Bestandteile des neuen Verständnisses der Interaktion zwischen dem Staat und seinen Bürgern. Einbeziehung und Mitwirkung sollen politische Entscheidungen legitimieren und die gesellschaftliche Betätigung in Selbstorganisationsprozessen stärken, während gleichzeitig Informationen, Wissen und Kompetenzen der Bürger in Verwaltungsentscheidungen Eingang finden (Mietzner et al., 2015, S. 20; Parycek, 2008, S. 5). Die Forderung nach Partizipation der Zivilgesellschaft wird auch immer wieder von NGOs artikuliert (Heggstadt \& Froystad, 2011, S. 24; Kühn \& Sherman, 2014, S. 16; U4 Anti-Corruption Resource Centre et al., 2012, S. 7).

Vor allem die IKT bietet neue Möglichkeiten und Verfahren zur Beteiligung, welche unter dem Begriff E-Partizipation zusammengefasst werden (Wimmer et al., 2015, S. 3). E-Partizipation umfasst die Beteiligung von natürlichen und juristischen Personen an Prozessen der Entscheidungsvorbereitung und -findung in Politik und Verwaltung unter Nutzung moderner IKT (Leitner, 2018, S. 12; Mietzner et al., 2015, S. 20; von Lucke, 2012b, S. 3 f.). Unter dem Begriff der offenen Partizipation werden die zeit- und ortsunabhängigen Formate der Bearbeitung, Gestaltung, Beratung, Diskussion, Entscheidung und Beauftragung 
mit Blick auf Problemdefinition, Agenda Setting und Entscheidungsfindung als Teil eines sechsstufigen Politprozesses verstanden, wie in Abbildung 6.18 zusammengefasst (von Lucke, 2012c, S. 5). In Ergänzung zur E-Partizipation steht bei der offenen Partizipation die möglichst zeitnahe und direkte Interaktion mit einem Netzwerk von Vielen im Vordergrund.

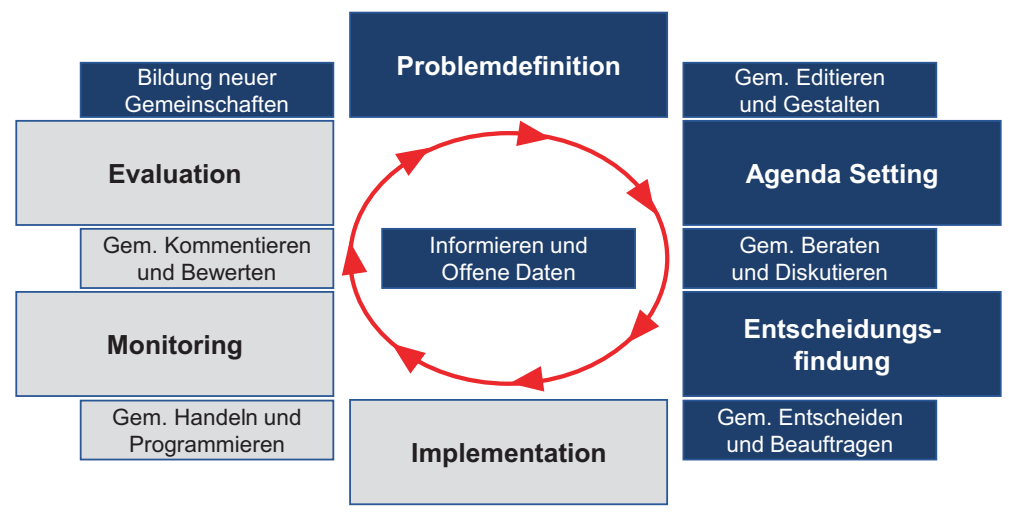

Abbildung 6.18 Schwerpunktbereiche einer offenen Partizipation - politisches Engagement (in Anlehnung an von Lucke, 2012c, S. 6)

Eine erste, grobe Abgrenzung von E-/offener Partizipation und E-/offener Kollaboration bietet Abbildung 6.19 an. Wichtig ist hier zunächst auch die Unterscheidung zwischen Partizipation und Kollaboration: Im Rahmen einer konkreten Entscheidungsfindung wird von Partizipation gesprochen, im Zuge einer entscheidungsunabhängigen Begleitung oder Lösungsfindung von Kollaboration. E-Partizipation/E-Kollaboration ist im Gegensatz zur offenen Partizipation/Kollaboration in der Regel zeit- und/oder ortsgebunden.

Als Stärken der E-Partizipation gelten die Möglichkeiten individualisierter und personalisierter Informationsangebote (zum Beispiel Anpassung des Newsletters auf eigene Interessensbereiche oder Spracheinstellungen) und das höhere Interaktivitäts- und Feedbackpotential. Chancen, die mit der E-Partizipation einhergehen, sind die Erreichbarkeit weiterer Zielgruppen, das Internet, welches viele Technologien integrieren kann, sowie die administrative Ent- 




Abbildung 6.19 Einordnung E-/offene Partizipation und E-/offene Kollaboration

lastung (Parycek, 2008, S. 14 ff.). Schwächen liegen vor allem darin, dass die Einbindung in den politischen Entscheidungsprozess mit entsprechender Rechtsverbindlichkeit gegeben sein muss, da sonst die Gefahr einer Symbolpolitik besteht (Parycek, 2008, S. 26). Zudem ist das mit E-Partizipation verbundene Engagement oftmals zufällig, inkonsistent, zeitlich unklar und nicht ausreichend (Cruickshank \& Ryan, 2015, S. 11 ff.). Meinungen und Argumente erfordern eine angemessene Moderation und die hierauf basierenden Entscheidungen müssen transparent und verständlich sein (Mietzner et al., 2015, S. 21; Parycek, 2008, S. 21). Ein Risiko kann sein, dass Internetnutzer gegebenenfalls nicht die Gesellschaft repräsentieren, da sie mehrheitlich weiß, männlich, um die 30 Jahre alt und internetaffin sind (Serdült et al., 2015, S. 27 f.; Wagner \& Schlögel, 2014, S. 216). Zudem ist möglich, dass sich Internetnutzer in eine Gruppe Gleichgesinnter zurückziehen, was den Austausch verschiedener Argumente verhindert und damit nicht dem demokratischen Gedanken entspricht (Wagner \& Schlögel, 2014, S. 218). Eventuell werden trotz der Beteiligung Vieler keine qualitativen Beiträge erzeugt. Außerdem fehlt oftmals die Nutzerorientierung (Parycek, 2008, S. 22 ff.). Tabelle 6.14 fasst Stärken, Schwächen, Chancen und Risiken zusammen. 
Tabelle 6.14 SWOT-Analyse E-Partizipation

\section{Stärken}

- Individualisierte und personalisierte Informationsangebote über z. B. Newsletter, FAQ, Sprache, Layouts, Nutzerprofile möglich

- Interaktivitäts- und Feedbackpotential

\section{Schwächen}

- Die Einbindung in den politischen Entscheidungsprozess mit entsprechender Rechtsverbindlichkeit ist eine Herausforderung

- Das Engagement ist oftmals eher zufällig, inkonsistent und zeitlich unklar, teilweise auch unzureichend

- Sicherstellung der Nachvollziehbarkeit der Ergebnisse

- Moderation und angemessene Kommunikation sind erforderlich, um zu tragbaren Ergebnissen zu gelangen

\section{Chancen}

\section{Risiken}

- Erreichung von zusätzlichen Zielgruppen (z. B. im Ausland lebende Deutsche)

- Repräsentanz der Mehrheit durch eine Minderheit

- Integration vieler Technologien im Internet (z. B. TV, Smartphones) und Formate

- Fehlende Nutzerorientierung

- Quantität versus Qualität der Beiträge (Zeitungsartikel, Podcasts, Videocasting als Sitzungs-Aufzeichnungen bzw. LiveÜbertragungen) bis hin zu spielerischen Elementen wie Planspielen

- Administrative Entlastung, über z. B. automatische Empfangsbestätigungen

\subsubsection{Erscheinungsformen und Anwendung im öffentlichen Einkauf}

Partizipation kann verschiedenartig kategorisiert werden: nach den Merkmalen formell/informell, Top-down/Bottom-up, direkt-demokratisch/beratend sowie nach Intensitätsstufen. Formell bedeutet, dass gesetzlich fixierte und standardisierte Verfahren genutzt werden und diese an eine verpflichtende Durchführung gebunden sind, während informelle Verfahren freiwillig, in ihrer Gestaltung freier und rechtlich nicht geregelt sind. So können informelle Verfahren auch auf die Erarbeitung eines gemeinsamen Ergebnisses ausgerichtet sein - wer wie teilnimmt, kann frei festgelegt werden. Top-down-Prozesse werden aus der Politik und Verwaltung initiiert, wohingegen Bottom-up-Prozesse durch die organisierte Öffentlichkeit, NGOs, Vereine oder Bürgerinitiativen angetrieben werden. Im Idealfall bedingen sich beide Varianten wechselseitig. Erstere unterliegen formellen Entscheidungsprozessen, Letztere besitzen eher einen informellen Charakter (Albrecht et al., 2008, S. 16; Parycek, 2008, S. 8). Direktdemokratische Formen sind jene, in denen der Bürger direkt abstimmen kann; 
deliberative Formen umfassen den Austausch von Argumenten in Diskussionen (Mietzner et al., 2015, S. 20). In Bezug auf Intensitätsstufen kann zum Beispiel nach Information (Newsletter, Sitzungsübertragungen etc.), Konsultation (Bürger-Panels, Chats, Diskussionsforen etc.), Kooperation (Online-Mediation, interaktive Planungen etc.) und Mitentscheidung (E-Voting, Online-Umfragen etc.) unterschieden werden (Parycek, 2008, S. 10).

Um aus Sicht des Nutzers partizipieren zu können, sind Interesse, Zugang und Medienkompetenz erforderlich. Der Zugang zum Internet muss zuerst gegeben sein. Das Interesse und die Akzeptanz bei den Nutzern können erhöht werden, indem die Angebote entsprechend beworben werden (Wagner \& Schlögel, 2014, S. 215 ff.). Medienkompetenz umfasst das Erlernen technischer Fähigkeiten, Grundlagenwissen über die Funktionsbedingungen des Internets, ein kritischer und reflektierter Umfang mit dem Internet und den dortigen Informationen, Datenschutz und eine angemessene Kommunikation im Netz (Netiquette). Dies kann als gesamtgesellschaftliche, aber insbesondere staatliche Aufgabe verstanden werden (Wagner \& Schlögel, 2014, S. 214 f.).

Mit Blick auf die Bereitsteller sorgt eine Multi-Kanalstrategie dafür, dass neben der Online-Kommunikation alternative Wege der Offline-Kommunikation bereitstehen, um so die Erreichbarkeit für unterschiedliche Zielgruppen zu gewährleisten (Barrierefreiheit) und gleichzeitig eine höhere Außenwirkung zu erzielen. Die Gewährleistung von Datenschutz und Informationssicherheit dient nicht nur der Einhaltung rechtlicher Vorschriften, sondern auch dem Aufbau von Vertrauen. Die erarbeiteten Entscheidungen müssen für die Beteiligten nachvollziehbar sein. $\mathrm{Zu}$ guter Letzt ist eine bestmögliche Einpassung in die bestehenden Strukturen unter Berücksichtigung von Benutzerfreundlichkeit anzustreben (Parycek, 2008, S. 18 ff.).

In Tabelle 6.15 sind mögliche Formate (Albrecht et al., 2008, S. 19 ff.) und ihre Zuordnung zu Intensitätsstufen (Information, Konsultation, Kooperation und Mitentscheidung) in den wesentlichen Anwendungen dargestellt. Nicht alle Formate decken alle Intensitätsstufen ab. Die meisten Formate sind auf der Stufe der Information als Basis für eine nachhaltige Beteiligung zu finden. Nur mit Transparenz können informierte Entscheidungen erfolgen. Auf der Stufe der Konsultation erfolgt dann die Rückkopplung und eine wechselseitige Kommunikation, dies kann auch in Kombination mit Offline-Angeboten erfolgen. Auf Ebene der Kooperation können die Beteiligten aktiv konkrete Projekte mitgestalten oder begleiten. Mitentscheidungen sind mithilfe von E-Voting und E-Petitionen möglich. 
Tabelle 6.15 Zuordnung der Beteiligungsformate zu Intensitätsstufen (in Anlehnung an Albrecht et al., 2008, S. 24 ff.; Parycek, 2008, S. 12)

\begin{tabular}{|l|c|c|c|c|}
\hline $\begin{array}{l}\text { Intensitätsstufen / } \\
\text { IKT-Formate Partizipation }\end{array}$ & $\begin{array}{c}\text { Infor- } \\
\text { mation }\end{array}$ & $\begin{array}{c}\text { Konsul- } \\
\text { tation }\end{array}$ & $\begin{array}{c}\text { Koope- } \\
\text { ration }\end{array}$ & $\begin{array}{c}\text { Entschei- } \\
\text { dung }\end{array}$ \\
\hline $\begin{array}{l}\text { Webseiten, Portale, RSS feed, } \\
\text { Audio/Video Podcast, Chat, } \\
\text { Instant Messaging }\end{array}$ & $\mathrm{x}$ & & & \\
\hline $\begin{array}{l}\text { Newsletter, Social tagging, } \\
\text { Ranking/Rating tool }\end{array}$ & $\mathrm{x}$ & $\mathrm{x}$ & \\
\hline $\begin{array}{l}\text { Online-Spiele und Simulatio- } \\
\text { nen, interaktive Karten, Web- } \\
\text { cast, Webblog, Telefon/ Call- } \\
\text { Center }\end{array}$ & $\mathrm{x}$ & $\mathrm{x}$ & $\mathrm{x}$ & \\
\hline $\begin{array}{l}\text { SMS, E-Mail, Mailing-Liste, } \\
\text { Videokonferenz, Online-Forum }\end{array}$ & $\mathrm{x}$ & $\mathrm{x}$ & $\mathrm{x}$ & \\
\hline $\begin{array}{l}\text { Feedbackformular, Online- } \\
\text { Befragung, Quick poll }\end{array}$ & & $\mathrm{x}$ & $\mathrm{x}$ & \\
\hline $\begin{array}{l}\text { Wiki, Soziale Netzwerke, Foto/ } \\
\text { Video Community }\end{array}$ & & & $\mathrm{x}$ & \\
\hline Virtuelle Welten & & & $\mathrm{x}$ \\
\hline E-Petition & & & & \\
\hline E-Voting & & & & \\
\hline
\end{tabular}

Zugleich muss man die Kombinatorik in Tabelle 6.15 vor dem Hintergrund des jeweiligen Kontextes (hier öffentlicher Einkauf) bewerten, also eine dreidimensionale Betrachtung durchführen. In Bezug auf den öffentlichen Einkauf könnten Beispiele entlang des Vergabeprozesses und der Intensitätsstufen wie in Tabelle 6.16 aussehen (in Klammern stehen mögliche Prozessstufen).

Tabelle 6.16 Beispiele für E-Partizipation im öffentlichen Einkauf (P: Planung, A: Ausschreibung, B: Bewertung, V: Vergabe, Df.: Durchführung)

\begin{tabular}{l|l}
\hline Intensitätsstufe & Beispiele E-Partizipation im öffentlichen Einkauf \\
\hline Information & $\begin{array}{l}\text { 1. Ankündigung eines Einkaufsplanungsprozesses über ein Portal oder } \\
\text { eine Mailingliste zwecks Teilnahme (P) }\end{array}$ \\
& $\begin{array}{l}\text { 2. Bereitstellung der Vergabeunterlagen und Bewertungskriterien } \\
\text { gegen einen speziellen/kostenpflichtigen Portalzugang (A) }\end{array}$ \\
& $\begin{array}{l}\text { 3. Mitteilung der Vergabeentscheidung an die Beteiligten per Mail (V) } \\
\text { per Mail (prozendung von Sitzungsergebnissen als Protokoll an die Beteiligten }\end{array}$ \\
\hline
\end{tabular}


Tabelle 6.16 (Fortsetzung)

\begin{tabular}{|c|c|}
\hline Intensitätsstufe & Beispiele E-Partizipation im öffentlichen Einkauf \\
\hline $\begin{array}{l}\text { Konsultation/ } \\
\text { Kooperation }\end{array}$ & $\begin{array}{l}\text { 5. Öffnung der Angebote in Anwesenheit der Bieter, Bereitstellung } \\
\text { eines Sitzungsprotokolls per Mail (B) } \\
\text { 6. Nutzung von Feedbackformularen auf Webseiten (prozess- } \\
\text { unabhängig) } \\
\text { 7. Zusammenarbeit/Entscheidungsvorbereitung über Auswertung von } \\
\text { Online-Erhebungen (prozessunabhängig) } \\
\text { 8. Kommunikationskanal für Hinweisgeber über eine auf dem Portal } \\
\text { hinterlegte Mailingliste oder ein Feedbackformular, zum Beispiel } \\
\text { Mexiko: https://testigosocial.mx oder Kanada: https://www.ic.gc.ca/ } \\
\text { eic/site/094.nsf/frm-eng/MBED-AJL22H (P, B, V) } \\
\text { 9. Einbindung von Vertretern der Zivilgesellschaft, Wirtschaft, Wissen- } \\
\text { schaft zur Herbeiführung einer Einkaufsentscheidung oder aber zur } \\
\text { Beteiligung bei Veränderungen von Verträgen über zum Beispiel } \\
\text { eine Mailingliste, Online-Befragung, Industrietage, Fokusgruppen, } \\
\text { persönliche Sitzungen (P, B, V), zum Beispiel Kanada: https:// } \\
\text { buyandsell.gc.ca/initiatives-and-programs/smart-procurement/ } \\
\text { about-smart-procurement (P, B, V) }\end{array}$ \\
\hline
\end{tabular}

Hieran sieht man, dass es jenseits der klassischen Beteiligungsformate (Mail, Einstellung von Unterlagen über Portale) durchaus weitere Beteiligungsmöglichkeiten im öffentlichen Einkauf entlang des gesamten Einkaufsprozesses gibt, deren Anwendung auch für Deutschland überprüft werden sollte.

Eine besondere Herausforderung ist, dass es sich beim öffentlichen Einkauf um ein zumeist komplexeres Verfahren handelt, das ein tieferes fachliches Verständnis voraussetzt. Es gilt zudem, die unterschiedlichen Korruptionsformen und ihre Investigationsmöglichkeiten, lokale Besonderheiten sowie sektorspezifische Gegebenheiten zu kennen. Um hier einen praktischen Ansatz zu finden, ist es möglich, sich zunächst beispielweise nur auf Teilprozesse des öffentlichen Einkaufs zu fokussieren, besonders korruptionsanfällige Sektoren zu priorisieren, nur hochwertige Einkaufsvorgänge zu untersuchen oder Projekte zu analysieren, auf die die Öffentlichkeit hingewiesen hat (de Simone \& Shah, 2012, S. 46 ff.).

Obwohl es zahlreiche Beispiele für Partizipation gibt, ist eine Bezifferung des Nutzens selten vorzufinden (OECD, 2007b). Mit dem „Social Witness Programm" in Mexiko und der Initiative "Smart Procurement" in Kanada können dennoch zwei erfolgreiche Beispiele für Partizipation in Bezug auf den öffentlichen Einkauf beschrieben werden, die mithilfe moderner IKT umgesetzt wurden. 


\section{„Social Witness Programm“ in Mexiko}

Das „Social Witness Programm“ in Mexiko (http://www.testigosocial.org. $\mathrm{mx} /$ ) besteht seit 2004 und sieht vor, dass ab einer gewissen Wertgrenze NGOs und Bürger als "Social Witnesses“ (Zeugen der Zivilgesellschaft) durch das Ministerium ausgewählt werden. Ihre Funktion ist es, Strategien zur Verbesserung der Transparenz, Objektivität und Einhaltung der rechtlichen Vorgaben vorzuschlagen und illegale Verhaltensweisen aufzuzeigen. Sie sind unabhängige und anerkannte Experten und ihre Rolle ist es, gemeinsam mit der einkaufenden Behörde die Ausschreibungsunterlagen zu erstellen und die Vergabeentscheidung zu treffen (de Simone \& Shah, 2012, S. 43 f.). Im Jahr 2010 beteiligten sich Zeugen der Zivilgesellschaft an über 120 öffentlichen Einkaufsverfahren (Sheppard \& Cantera, 2012, S. 37). Zahlen lassen sich beispielhaft für den Flughafen Mexiko und den Zeitraum 2016 bis 2017 nennen: Das zugewiesene Budget der registrierten Verträge lag bei 151.933,7 Millionen US-Dollar. Der Gesamtbetrag, der vergeben wurde, lag schließlich bei 125.786,9 Millionen US-Dollar (Transparencia Mexicana, 2019). Ein weiteres Beispiel ist jenes für den Einkauf der Bundeskommission für Elektrizität: Hier führte das Programm zu Einsparungen in Höhe von 26 Millionen US-Dollar (Kühn \& Sherman, 2014, S. 29; OECD, 2007b, S. 118). Insgesamt konnten die Einkaufskosten gesenkt und die Zahl der Teilnehmer an öffentlichen Ausschreibungen erhöht werden (de Simone \& Shah, 2012, S. 43).

\section{„Smart Procurement"“ in Kanada}

Kanadas „Smart Procurement“-Ansatz besteht aus vier Bestandteilen: frühes Engagement, effektive Steuerung, unabhängiger Rat und Vorteile für Kanadier. Ziel ist es, die bereitgestellten Dienstleistungen für die kanadische Bevölkerung zu verbessern. Die Initiative wird vom Public Works and Government Services Canada (PWGSC) im Office of Small and Medium Enterprises (OSME) geleitet. Diese ist wiederum bei der Behörde Public Services and Procurement Canada (PSPC) angegliedert.

Unter frühem Engagement versteht man, dass man einen frühzeitigen und stetigen Dialog zwischen Industrie, Kunden und PWGSC über den gesamten Einkaufsprozess etabliert, der bereits mit der Feststellung der Bedarfe beginnt. Dies soll dazu führen, Risiken zu minimieren, innovative Lösungen zu identifizieren, Sicherheitsanforderungen und andere Einflüsse auf die Projektzeitleiste zu erfahren, Erwartungen zu schärfen und einen stärkeren Wettbewerb $\mathrm{zu}$ fördern. Als mögliche Formen des frühen Engagements kommen zum Beispiel Informationsanfragen, Industrietage, informelle Diskussionen, Online-Befragungen, Online-Kollaborationstools, Fokusgruppen und individuelle 
Konsultationen mit Lieferanten in Betracht. Effektive Steuerung setzt die Spielregeln für die Interaktionen zwischen Anforderern und Lieferanten. Dies beinhaltet zum Beispiel die Schaffung von projektspezifischen Strukturen, um so einen fortwährenden Dialog sicherzustellen, genauso wie die Planung und Kontrolle von Aktivitäten und Ressourcen. Dies kann zum Beispiel in der Praxis über die Etablierung eines Sekretariats oder die Absprache von Regeln des Miteinanders zwischen allen Anspruchsgruppen zur Kommunikation erfolgen. Unabhängiger Rat über den gesamten Einkaufsprozess soll sicherstellen, dass neutrale Dritte die Einkaufsaktivitäten in Form von Expertise, Validierung und allgemeinen Rat kommentieren, um so Einkaufsentscheidungen zu optimieren. Unter Vorteilen für Kanadier wird verstanden, dass durch die Anwendung der zuvor beschrieben Mechanismen ein Motor für eine ökonomische Entwicklung geschaffen wird, deren Resultate über einen optimierten Steuermitteleinsatz bei den Kanadiern wieder ankommen (Public Works and Government Services Canada (PWGSC), 2019).

Der Ansatz zeigt sehr fortschrittlich, wie partizipative Bestandteile im öffentlichen Einkaufsprozess integriert werden können. Leider gibt es keine frei zugänglichen Erhebungen zu den Wirkungen und Ergebnissen dieser Ansätze.

\subsubsection{Kollaboration als Organisation Vieler zur Einflussnahme auf Wirtschaft und Politik}

\subsubsection{Definition}

Bei Kollaboration ,geht es um die koordinierte Zusammenarbeit von Personen oder Organisationseinheiten zur Erreichung von gemeinsam vereinbarten Zielen“ (von Lucke, 2012c, S. 2). Von Lucke spricht in dem Zusammenhang von Input, Outcome, Output und Impact: Ressourcen wie zum Beispiel Wissen oder Kapital werden eingebracht, erzeugen ein Ergebnis (Output), haben gesellschaftliche Auswirkungen (Outcome) und bewirken konkrete Veränderungen (Impact). E-Kollaboration umfasst die elektronischen Formen der Zusammenarbeit, Offene Kollaboration beinhaltet darüber hinaus alle offenen Formen der Zusammenarbeit (von Lucke, 2012c, S. 2). Kollaboration setzt in Abgrenzung zur Partizipation nach einer bereits erfolgten Entscheidung in der Implementierung, beim Monitoring von Ergebnissen und der Evaluierung an (von Lucke, 2012c, S. 1); zielt also nicht auf eine Entscheidung ab (Harrison et al., 2012, S. 88). Abbildung 6.20 illustriert die mit Kollaboration verbundenen Bereiche. 


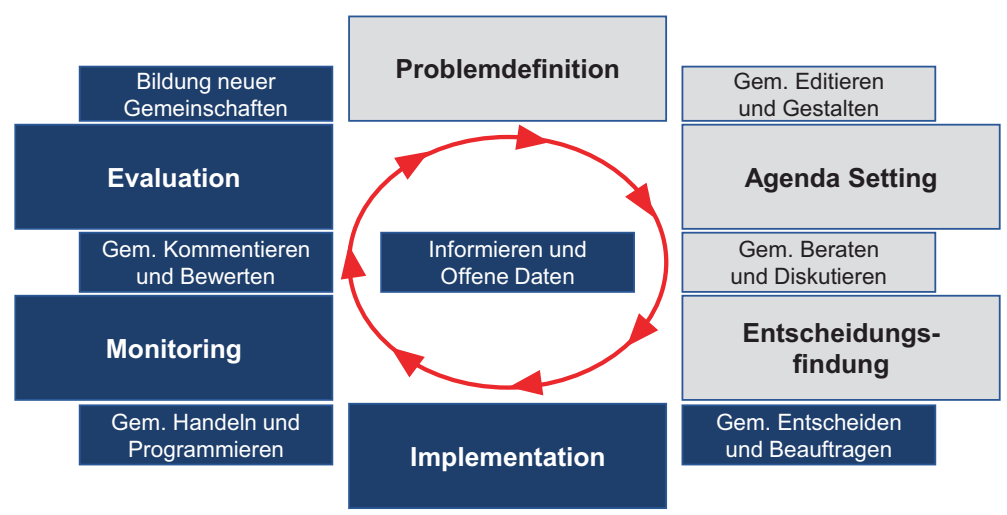

Abbildung 6.20 Schwerpunktbereiche einer offenen Zusammenarbeit - gesellschaftliches Engagement (in Anlehnung an von Lucke, 2012c, S. 6)

Kollaboration betrifft nicht nur die inner- oder interbehördliche, sondern auch die Zusammenarbeit mit externen Akteuren (Janda, 2012, S. 17). Die moderne IKT, beispielsweise E-Mail, Kollaborationsplattformen, Online-Chats etc. erleichtern die Zusammenarbeit (Janda, 2012, S. 26). Da es in den Verwaltungen oftmals an Ressourcen fehlt, kann über die Auslagerung bestimmter Themen (Outsourcing) beziehungsweise Einbeziehung des Wissens von außen (Crowd Sourcing) auf ein zusätzliches Netzwerk an Freiwilligen zurückgegriffen werden (von Lucke, 2012c, S. 3). Die Erwartung ist, dass durch eine offene Zusammenarbeitsform und die Integration vieler Dritter in den politischen Prozess neue und innovative Lösungsansätze für bestehende Probleme gefunden werden können (Edelmann et al., 2012, S. 27; Harrison et al., 2012, S. 89).

Die Hürden sind vergleichbar mit jenen der Partizipation (von Lucke, 2012c, S. 36). Die Wichtigsten in diesem Kontext sind, dass das Bereitstellen von Technologie für eine gelungene Kollaboration nicht ausreicht und dass die Ausrichtung in der Bereitstellung von Service-Infrastrukturen an der Technologie und nicht an den Bedürfnissen der Nutzer erfolgt (Edelmann et al., 2012, S. 25).

Somit wird diese informelle, nicht-hierarchische Art der Kollaboration mit einem breiteren Netzwerk noch nicht vollständig durch öffentliche Verwaltungen genutzt. Die Herausforderungen liegen in der Definition eines Rahmens, der sowohl die Governance, die Rollen wie auch Technologien stimmig zusammenbringt (Edelmann et al., 2012, S. 22). Kollaboration erfüllt dabei keinen Selbstzweck, sondern sollte zielgerichtet eingesetzt werden (Harrison et al., 2012, S. 89). 
Es gibt bislang nur wenige Ansätze in der Literatur zur Konzeptualisierung beziehungsweise Messung des Nutzens eingesetzter Kollaborationsformen. Gute Beispiele sind Public Value Frameworks, die versuchen, die Erzeugung eines öffentlichen Nutzens in einem Gesamtbild darzustellen (Edelmann et al., 2012, S. 27; Harrison et al., 2012, S. 89 ff.) oder die Beschreibung der Effektivität von Netzwerken in der Erzeugung von Dienstleistungen (Provan \& Milward, 2001, S. 414). Allerdings bedarf es hier noch weiterer wissenschaftlicher Begleitung, um die Abgrenzung und Mehrwerte des Begriffs Kollaboration gegenüber anderen Zusammenarbeitsformen klarer herauszustellen (Wewer, 2014, S. 38 ff.).

Tabelle 6.17 fasst die Stärken, Schwächen, Chancen und Risiken noch einmal abschließend zusammen.

Tabelle 6.17 SWOT-Analyse E-Kollaboration

\begin{tabular}{|c|c|}
\hline Stärken & Schwächen \\
\hline $\begin{array}{l}\text { - Ausgereifte technische Lösungen sind als } \\
\text { weiterer Kommunikations- und Kollabo- } \\
\text { rationskanal nutzbar } \\
\text { - Nutzung der vorhandenen technischen } \\
\text { Lösungen für die innerbehördliche sowie } \\
\text { externe Zusammenarbeit }\end{array}$ & $\begin{array}{l}\text { - Begriffliche Unschärfen } \\
\text { - Politische Programme, aber auch Online- } \\
\text { Portale, besitzen oft keine Kollaborations- } \\
\text { strategie und -möglichkeit } \\
\text { - Abwägung, wo der Einsatz von Kollabo- } \\
\text { ration sinnvoll ist } \\
\text { - Verantwortlichkeit, Verbindlichkeit und } \\
\text { Dauerhaftigkeit des Engagements sind } \\
\text { oftmals unklar }\end{array}$ \\
\hline Chancen & Risiken \\
\hline $\begin{array}{l}\text { Durch die Nutzung von IKT in der } \\
\text { Zusammenarbeit können Ideen besser } \\
\text { ausgetauscht und weiterentwickelt werden }\end{array}$ & $\begin{array}{l}\text { - Repräsentanz der Mehrheit durch eine } \\
\text { Minderheit } \\
\text { - Fehlende Erkenntnis, dass das Bereitstel- } \\
\text { len von Technologie allein nicht ausreicht } \\
\text { und damit Risiko einer unzureichenden } \\
\text { Nutzung } \\
\text { - Finden geeigneter Partner } \\
\text { - Qualität der Beiträge und Leistungen }\end{array}$ \\
\hline
\end{tabular}

\subsubsection{Erscheinungsformen und Anwendung im öffentlichen Einkauf}

$\mathrm{Zu}$ Zeiten des Web 1.0 fand Kollaboration oftmals persönlich zwischen Verwaltung und einzelnen Akteuren oder in Form von Wahlveranstaltungen statt (,one to many“) (von Lucke, 2012c, S. 3). Gemäß der Web 2.0-Logik wird der öffentliche Mehrwert nicht mehr allein von den Verwaltungen erzeugt, sondern durch deren Zusammenarbeit mit verschiedenen Akteuren (Wirtschaft, Bürger etc.) mithilfe diverser 
asynchroner (Blogs, Wikis etc.) und synchroner Formate (Chats, Videokonferenzen etc.). Auch Whiteboards für das Teilen von Applikationen, gemeinsame Plattformen, Portale und Shared Service Center sind zu nennen. Hierdurch entsteht eine Unabhängigkeit von Zeit und Raum (u. a. Janda, 2012, S. 24; von Lucke, 2012c, S. 3 f.). Ein Ziel dieser Zusammenarbeitsformen ist die gemeinsame Abstimmung von Maßnahmen, das Erreichen eines Konsenses und die Dokumentation von Tätigkeiten und Erkenntnissen (Lambropoulos et al., 2009, S. 92).

Eine Besonderheit der Zusammenarbeit ist die zumeist lose verbundene Organisation Einzelner, die sich die Mittel der IKT zunutze machen, um gemeinsam Einfluss auf Politik und Wirtschaft zu nehmen (,,many to many“). Auch wenn es früher bereits die Zusammenarbeit der Verwaltung mit Interessensverbänden oder Gewerkschaften gab, waren dies dennoch partikuläre Interessensvertretungen - die Möglichkeiten der Kollaboration mit einer Vielzahl von Akteuren im Sinne eines Netzwerks ergibt sich erst durch die weiterentwickelten IKT (Edelmann et al., 2012, S. 27). Diese Art der Koproduktion wird sich fortsetzen, je mehr der Zugang zu Applikationen, Datenbanken und Wissen geöffnet wird und sich die Fähigkeiten entwickeln, hiermit umzugehen. Die reine Bereitstellung der Technologie wird dabei nicht genug sein: Es muss sichergestellt werden, dass die Anwender positive (Online-)-Erfahrungen und Interaktionen haben. Das bedeutet, dass Kollaboration nicht einfach geschieht, sondern durchdacht, kanalisiert und begleitet werden muss (Edelmann et al., 2012, S. 24).

Die Anwendung auf den öffentlichen Einkauf kann an der Schnittstelle zur Öffentlichkeit, aber auch nach innen gerichtet erfolgen. „Nach innen“ bedeutet: auf organisatorische Kooperationsformen wie beispielsweise Shared Service Center, zentrale Vergabestellen oder gemischtwirtschaftliche Gesellschaften gerichtet (siehe Abschnitt 6.3.1) (Bakker et al., 2008, S. 3 ff.). Als Vorteile einer Kooperation im öffentlichen Einkauf werden verbesserte Effizienzen und eine höhere Effektivität genannt. Erstere können durch die Reduktion von Transaktionskosten, die Bündelung von Einkaufsaktivitäten und das Erzielen von Skaleneffekten erreicht werden, während eine verbesserte Effektivität durch Qualitätsverbesserungen, optimierte Prozessabläufe und ein verbessertes Lieferantenmanagement erzielt werden kann. Die Nachteile werden in einer Erhöhung der Komplexität des Einkaufsprozesses sowie Koordinationsaufwänden gesehen, aber auch in einem Verlust an Flexibilität und vollständiger Kontrolle (Bakker et al., 2008, S. 1; Harland et al., 2017, S. 3 ff.). Die vielfältigen Einsatzmöglichkeiten einer Kooperation können somit zwischenbehördlich ebenfalls durch den Einsatz von IKT verbessert werden. Hier kann die elektronische Kollaboration ebenfalls Akzente setzen. Diese werden beispielhaft in Tabelle 6.18 aufgezeigt. 
Tabelle 6.18 Beispiele für E-Kollaboration im öffentlichen Einkauf (in Klammern stehen mögliche Prozessstufen; P: Planung, A: Ausschreibung, B: Bewertung, V: Vergabe, Df.: Durchführung)

\begin{tabular}{l|l}
\hline Intensitätsstufe & Beispiele E-Kollaboration im öffentlichen Einkauf \\
\hline Information & $\begin{array}{l}\text { 1. Bereitstellung von Statusberichten, zum Beispiel aus Steering } \\
\text { Committees, über ein Portal an die Beteiligten (Df.) }\end{array}$ \\
\hline Konsultation/ & $\begin{array}{l}\text { 2. Wissensmanagement mittels Etablierung von Fachnetzwerken, } \\
\text { Schulungsangeboten etc. über Portale, Blogs, Webinare, zum } \\
\text { Beispiel Deutschland: Das DVNW (https://www.dvnw.de/) oder } \\
\text { KOINNO (https://www.koinno-bmwi.de/) (prozessunabhängig) } \\
\text { 3. Kommunikationskanal für Hinweisgeber über eine auf dem Portal } \\
\text { hinterlegte Mailingliste oder ein Feedbackformular, zum Beispiel } \\
\text { Mexiko: https://testigosocial.mx oder Kanada: https://www.ic.gc.ca/ } \\
\text { eic/site/094.nsf/frm-eng/MBED-AJL22H (P, B, V) } \\
\text { 4. Zusammenarbeit über verschiedene Formate wie Hackdays, } \\
\text { Workshops, zum Beispiel Deutschland (NRW): https://open.nrw/ } \\
\text { zusammenarbeit (prozessunabhängig) }\end{array}$ \\
\hline
\end{tabular}

Mit Blick auf E-Kollaboration können zum Beispiel Ansätze wie das Deutsche Vergabenetzwerk (DVNW) sowie das KOINNO genannt werden.

Das Deutsche Vergabenetzwerk (https://www.dvnw.de/) ist ein internetbasiertes, kostenfreies Portal zur Information, zum Wissensaustausch und Diskurs $\mathrm{zu}$ allen Themen des Vergaberechts, aber darüberhinausgehend auch E-Government, öffentlichem Einkauf, Nachhaltigkeit und Sicherheit. Es wendet sich an Experten und Entscheider im Vergaberecht und öffentlichem Sektor und bildet so ein Netzwerk aus Mitgliedern der Verwaltung, Wirtschaft, Rechtspflege, Wissenschaft und Politik möglichst aller föderalen Ebenen. Neben dem internetbasierten Portal gibt es Regionalgruppen, den Vergabenetzwerk-Blog mit aktuellen Themen sowie den Deutschen Vergabetag. Das Portal verstärkt hier den persönlichen, klassischen Kontakt.

Als Inkubator rund um Innovationen im öffentlichen Einkauf kann KOINNO beim Bundesministerium für Wirtschaft und Energie angeführt werden. Über die Webseite https://www.koinno-bmwi.de erfolgt der Zugang zum Leistungsangebot, welches sich von Beratung über Veranstaltungen, Informationen bis hin zur Weiterbildung erstreckt. Neben Praxisbeispielen, einer Toolbox, Publikationen, E-Learning und einem Newsletter werden auch Konferenzen und Seminare angeboten. Auch hier erfolgt die Kombination klassischer Kommunikationsformen mit IKT. 


\subsection{Zwischenfazit: Fortgesetzte Intransparenz im Unterschwellenbereich in Deutschland}

Der Unterschwellenbereich des öffentlichen Einkaufs steht für den größten Teil der öffentlichen Ausgaben, ist aber im Vergleich zum Oberschwellenbereich mit seinen Berichtspflichten sehr intransparent. So gibt es keine einheitlichen Mechanismen zur Erhebung und Auswertung öffentlicher Einkaufsdaten für die Bundesrepublik Deutschland insgesamt ${ }^{25}$, geschweige denn für die Verteilungen der öffentlichen Vergaben nach Anzahl und Volumina auf den Oberschwellenund Unterschwellenbereich, getrennt nach Verfahrensarten oder föderalen Ebenen. Auch das im Jahr 2014 gestartete Projekt zur einheitlichen Vergabestatistik eignet sich nicht, um flächige Transparenz im Unterschwellenbereich herzustellen, da man sich auf lediglich fünf bereitzustellende Informationen beschränkt, in denen noch nicht einmal der finale Lieferant enthalten ist.

Obschon das Vergaberecht als Regelverfahren die öffentliche Ausschreibung und die beschränkte Ausschreibung mit Teilnahmewettbewerb vorsieht, wird dies nur in bis maximal einem Drittel der Verfahren angewendet - somit ist die Regel die Ausnahme.

Die Vergabeordnungen als Teil des Vergaberechts sehen unterschiedliche Pflichten zur Informationsbereitstellung im Allgemeinen und zur Auftrags- und Vergabebekanntmachung im Besonderen vor. Während die Auftragsbekanntmachung nur bei öffentlichen Ausschreibungen und jenen mit Teilnahmewettbewerb gilt, ist die Vergabebekanntmachung nur bei jenen Verfahren vonnöten, die ohne Teilnahmewettbewerb und erst ab einer bestimmten Größenordnung durchgeführt werden. Folglich ist es derzeit also schon aus gesetzlich-strukturellen Gründen nicht möglich, anhand der Bekanntmachungen eine Gesamtschau auf alle geplanten und erfolgten Ausschreibungen/Vergaben zu erhalten. Ein vergleichender Blick auf die zu veröffentlichenden Daten zeigt, dass die Auftragsbekanntmachung sehr viele Daten enthält (siehe Tabelle 6.8), die Vergabebekanntmachung nur sehr wenige Daten (siehe Tabelle 6.9). Auch dies schränkt die Auswertungsmöglichkeiten erheblich ein ex-ante soll der Wettbewerb gefördert werden, ex-post ist lediglich eine Minimalberichtspflicht zu erfüllen. Dies belegt aber auch, dass sich rein am operativen Prozess orientiert wird, nicht am möglichen strategischen Nutzen, den man aus der Erhebung

\footnotetext{
${ }^{25}$ Wie in Abschnitt 6.1.1 dargelegt, gehen die jüngsten Schätzungen aus 2016 von 348,5 Milliarden Euro pro Jahr aus (Schaupp \& Eßig, 2016, S. 51).
} 
und Auswertung dieser Daten gewinnen könnte. Allerdings lassen die Vergabeordnungen es durchaus zu, über den Rahmen der verpflichtend zu veröffentlichenden Daten hinauszugehen - es gibt im Vergaberecht keine Veröffentlichungsverbote, was der Öffnung öffentlicher Einkaufsdaten dienen kann (unter Berücksichtigung der Vorschriften anderer Rechtsgebiete, siehe Abschnitt 7.3.5 und 7.1).

Mit der spätestens ab 01. Januar 2020 geltenden Pflicht zur eVergabe wird die Hoffnung verbunden, dass auch im Unterschwellenbereich bald eine fundierte elektronische Datengrundlage vorliegen wird. Allerdings wird nach wie vor nur ein Teilbereich des öffentlichen Einkaufs elektronisch verfügbar sein: die Ausschreibung und ein Teil der Vergabe und dies lediglich für Liefer- und Dienstleistungen. Der Baubereich bleibt vollständig außen vor.

Der Nutzen der Öffnung wird vor allem in einer transparenteren Gestaltung des öffentlichen Einkaufs und in der Korruptionsbekämpfung gesehen. Dabei muss beachtet werden, dass Korruption ein vielschichtiges und interdependentes Problem ist, welches sich flexibel seiner Umgebung anpasst und daher einen Werkzeugkasten unterschiedlichster Maßnahmen erfordert. Der Mitteleinsatz hierfür muss sich am zu erwartenden Nutzen orientieren. Unterstützend kann in diesem Zusammenhang die Beteiligung Dritter im Entscheidungsprozess (Partizipation) sowie in der Lösungsfindung und Umsetzung (Kollaboration) sein. Die IKT bietet hier eine Vielzahl von Einsatzoptionen entlang des gesamten Einkaufsprozesses, die heute allerdings nur begrenzt genutzt werden (im Wesentlichen in der Bereitstellung von Vergabeunterlagen und im Austausch per Mail). Interessante Anregungen wurden mit Bezug auf Mexiko, Kanada, aber auch Deutschland aufgezeigt.

Nachdem in diesem Kapitel der Status quo des öffentlichen Einkaufs beschrieben wurde, dient Kapitel 7 dazu, aufzuzeigen, auf welchen bestehenden Rahmenbedingungen für eine Öffnung des öffentlichen Einkaufs aufgesetzt werden kann. 
Open Access Dieses Kapitel wird unter der Creative Commons Namensnennung 4.0 International Lizenz (http://creativecommons.org/licenses/by/4.0/deed.de) veröffentlicht, welche die Nutzung, Vervielfältigung, Bearbeitung, Verbreitung und Wiedergabe in jeglichem Medium und Format erlaubt, sofern Sie den/die ursprünglichen Autor(en) und die Quelle ordnungsgemäß nennen, einen Link zur Creative Commons Lizenz beifügen und angeben, ob Änderungen vorgenommen wurden.

Die in diesem Kapitel enthaltenen Bilder und sonstiges Drittmaterial unterliegen ebenfalls der genannten Creative Commons Lizenz, sofern sich aus der Abbildungslegende nichts anderes ergibt. Sofern das betreffende Material nicht unter der genannten Creative Commons Lizenz steht und die betreffende Handlung nicht nach gesetzlichen Vorschriften erlaubt ist, ist für die oben aufgeführten Weiterverwendungen des Materials die Einwilligung des jeweiligen Rechteinhabers einzuholen.

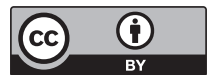

